Supporting Information

\title{
Ligand Controlled Ir-Catalyzed Regiodivergent Oxyamination of Unactivated Alkenes
}

Honghui Lei, John H. Conway, Jr., Caleb C. Cook, and Tomislav Rovis*

Department of Chemistry, Columbia University, New York, New York 10027

1. General Information $\quad 52$

2. Synthesis of alkenyl N-pivaloylhydroxamate $\quad$ S2

3. Synthesis of cationic $\operatorname{Ir}($ III) catalyst $\quad$ S6

4. Synthesis of $y$-lactams $\quad 57$

5. Synthesis of $\delta$-lactams $\quad 513$

6. Synthesis of $\gamma$ and $\delta$-lactones $\quad \$ 19$

7. Mechanistic studies $\quad 521$

8. X-Ray Crystal Structure $\$ 23$

9. References $\quad S 24$

10. NMR Spectra $\quad$ S25 


\section{General Information}

All reactions were performed in air using oven-dried glassware with magnetic stirring unless otherwise noted. Anhydrous solvents and reagents were purchased from Sigma Aldrich and used without further purification. Flash chromatography was conducted either manually on SiliCycle ${ }^{\circledR}$ SilicaFlash ${ }^{\circledR}$ P60 (230-400 mesh) silica gel or automatically via a Teledyne Isco Lumen CombiFlash with RediSep Rf Disposable Flash columns. Thin layer chromatography (TLC) was performed on Silicycle $250 \mu \mathrm{m}$ silica gel $60 \AA ̊$ plates. Visualization was accomplished with UV light (254 nm), lodine, or phosphomolybdic acid.

${ }^{1} \mathrm{H}$ NMR and ${ }^{13} \mathrm{C}$ NMR spectra were collected in $\mathrm{CDCl}_{3}$ or $\mathrm{CD}_{3} \mathrm{CN}$ on a Bruker $400 \mathrm{MHz}$ or 500 $\mathrm{MHz}$ spectrometers at ambient temperature. Chemical shifts $(\delta)$ are reported in parts per million (ppm), coupling constants $(\mathrm{J})$ are reported in $\mathrm{Hz}$, and multiplicity is described using the following abbreviations: $\mathrm{s}=$ singlet, $\mathrm{d}=$ doublet, $\mathrm{t}=$ triplet, $\mathrm{q}=$ quartet, $\mathrm{m}=$ multiplet, $\mathrm{br}=$ broad, or combinations thereof. All given ${ }^{13} \mathrm{C}$ NMR spectra peaks are singlets unless otherwise noted. Regioisomeric ratios were measured by integration of ${ }^{1} \mathrm{H}$ NMR spectra of product mixtures prior to purification. Low resolution mass spectra were recorded on a Waters Acquity H uPLC-MS. High resolution mass spectra (HRMS) were obtained from the Columbia University Chemistry Department Mass Spectrometry Facility on a Waters XEVO G2XS QToF mass spectrometer equipped with a UPC2 SFC inlet and a LockSpray source with one of the following three probes: electrospray ionization (ESI) probe, atmospheric pressure chemical ionization (APCI) probe, or atmospheric pressure solids analysis probe (ASAP). Infrared spectra were collected on a Perkin Elmer Spectrum Two FT-IR Spectrometer.

Carboxylate and halide nucleophiles 1a-10 were all purchased from Sigma Aldrich and used without further purification. Alkenyl N-pivaloylhydroxamate were either synthesized according to literature reports $\left(\mathbf{1} \mathbf{a}-\mathbf{1} \mathbf{c}^{1}, \mathbf{1}^{1}, \mathbf{1} \mathbf{k}^{1}\right)$, or general methods shown below (1d-1i). Alkenyl carboxylic acids were either purchased from Sigma Aldrich and used without further purification $\mathbf{( 5 a}, \mathbf{5 f})$ or synthesized according to literature reports $\left(\mathbf{5 b}-\mathbf{5} \mathbf{e}^{2}\right)$. Iridium cationic catalysts were synthesized according to the general method shown below.

\section{Synthesis of alkenyl $\mathrm{N}$-pivaloylhydroxamate}

\section{General Procedure A:}

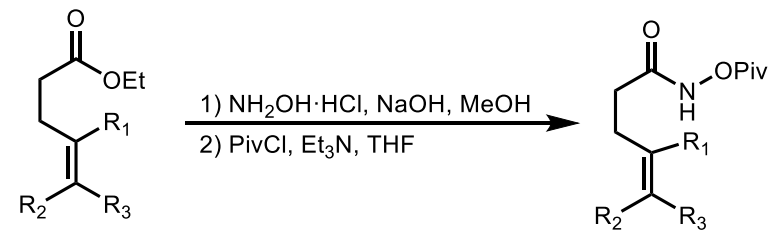

1

Alkenyl ethyl ester (1.0 equiv) was added to a suspension of $\mathrm{NH}_{2} \mathrm{OH} \cdot \mathrm{HCl}$ (20 equiv) and $\mathrm{NaOH}$ ( 40 equiv) in $\mathrm{MeOH}(0.2 \mathrm{M})$ at $0{ }^{\circ} \mathrm{C}$. The mixture was allowed to warm to room temperature, stirring overnight. The reaction was then acidified to $\mathrm{pH}=3$ with $1 \mathrm{~N} \mathrm{HCl}$. After removal of $\mathrm{MeOH}$ by rotary evaporation, the aqueous layer was extracted 4 times with EtOAc, the combined organic solution was dried over anhydrous $\mathrm{Na}_{2} \mathrm{SO}_{4}$, solids were filtered, and the filtrate was concentrated. The crude hydroxamate product was directly used in next step without further purification. 
The crude hydroxamate was dissolved in $\operatorname{THF}(0.2 \mathrm{M})$ at $0{ }^{\circ} \mathrm{C}$, followed by addition of $\mathrm{Et}_{3} \mathrm{~N}(1.5$ equiv.) and $\mathrm{PivCl}$ (1.1 equiv). The reaction was allowed to warm to room temperature, stirring until completed conversion. After that, the mixture was diluted with EtOAc, and washed with water and brine. The organic layer was then dried over anhydrous $\mathrm{Na}_{2} \mathrm{SO}_{4}$, solids were filtered, and the filtrate was concentrated by rotary evaporation. The crude mixture was purified by column chromatography.

\section{General Procedure B:}

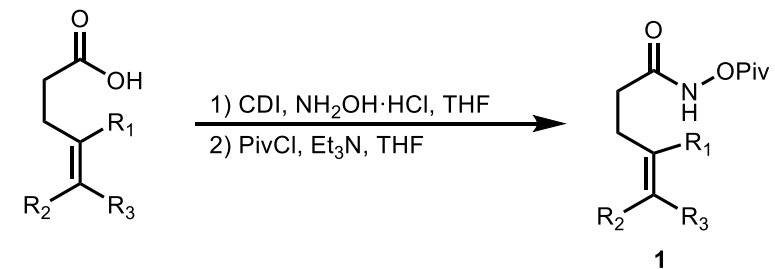

To a stirred solution of alkenyl carboxylic acid (1.0 equiv) in THF $(0.2 \mathrm{M})$ was added $1,1^{\prime}$-carbonyldiimidazole (1.5 equiv) at room temperature. Hydroxylamine hydrochloride was then added after bubbling ceased. The mixture was stirred overnight. The reaction was diluted with EtOAc, and washed with water and brine. The organic layer was then dried over anhydrous $\mathrm{Na}_{2} \mathrm{SO}_{4}$, solids were filtered, and the filtrate was concentrated by rotary evaporation. The crude hydroxamate product was directly used in next step without further purification.

The crude hydroxamate was dissolved in $\operatorname{THF}(0.2 \mathrm{M})$ at $0{ }^{\circ} \mathrm{C}$, followed by addition of $\mathrm{Et}_{3} \mathrm{~N}(1.5$ equiv.) and $\mathrm{PivCl}$ (1.1 equiv). The reaction was allowed to warm to room temperature, stirring until completed conversion ( 1h). After that, the mixture was diluted with EtOAc, and washed with water and brine. The organic layer was then dried over anhydrous $\mathrm{Na}_{2} \mathrm{SO}_{4}$, solids were filtered, and the filtrate was concentrated by rotary evaporation. The crude mixture was purified by column chromatography.<smiles>C/C=C/CCC(=O)NOCCO</smiles>

(E)-N-(pivaloyloxy)hex-4-enamide (1d)

(Prepared according to the general procedure $\mathbf{A}$ from ethyl (E)-4-hexenoate synthesized according to a literature procedure. $\left.{ }^{3}\right)$

Yield: $62 \%$. White solid. $\mathbf{R}_{f}=0.75$ (hexane/EtOAc 1:1).

${ }^{1} \mathrm{H}$ NMR $\left(500 \mathrm{MHz}, \mathrm{CDCl}_{3}\right) \delta 9.21(\mathrm{~s}, 1 \mathrm{H}), 5.56-5.48(\mathrm{~m}, 1 \mathrm{H}), 5.47-5.39(\mathrm{~m}, 1 \mathrm{H}), 2.38-2.28(\mathrm{~m}$, $4 \mathrm{H}), 1.65$ (dd, J = 6.2, $1.4 \mathrm{~Hz}, 3 \mathrm{H}), 1.31(\mathrm{~s}, 9 \mathrm{H})$.

${ }^{13} \mathrm{C}$ NMR $\left(126 \mathrm{MHz}, \mathrm{CDCl}_{3}\right) \delta 176.6,171.0,128.9,126.8,38.3,33.0,27.8,27.0,17.9$.

IR (neat, $\mathrm{cm}^{-1}$ ) v 3183, 2970, 1780, 1669, 1481, 1368, 1269, 1082, 966, 874

LRMS (ESI+APCI) m/z $\left[\mathrm{C}_{11} \mathrm{H}_{20} \mathrm{NO}_{3}\right]^{+}\left([\mathrm{M}+\mathrm{H}]^{+}\right)$calculated 214.1, found 214.2. 
$\int_{1}^{\mathrm{O}} \mathrm{H}^{\text {-OPiv }}$

(Z)-N-(pivaloyloxy)hex-4-enamide (1e)

(Prepared according to the general procedure A from ethyl (Z)-4-hexenoate synthesized according to a literature procedure. ${ }^{4}$ )

Yield: $59 \%$. White solid. $\mathbf{R}_{f}=0.75$ (hexane/EtOAc 1:1).

${ }^{1} \mathrm{H}$ NMR (500 MHz, CDCl $) \delta 8.99$ (brs, 1H), $5.59-5.51(\mathrm{~m}, 1 \mathrm{H}), 5.43-5.36(\mathrm{~m}, 1 \mathrm{H}), 2.44(\mathrm{q}, \mathrm{J}=$ $7.1 \mathrm{~Hz}, 2 \mathrm{H}), 2.32(\mathrm{t}, \mathrm{J}=7.7 \mathrm{~Hz}, 2 \mathrm{H}), 1.64(\mathrm{ddt}, \mathrm{J}=6.8,1.8,0.9 \mathrm{~Hz}, 3 \mathrm{H}), 1.32(\mathrm{~s}, 9 \mathrm{H})$.

${ }^{13} \mathrm{C}$ NMR $\left(126 \mathrm{MHz}, \mathrm{CDCl}_{3}\right) \delta 176.7,171.0,127.9,126.0,38.3,32.9,27.0,22.3,12.8$.

IR (neat, cm ${ }^{-1}$ ) v 3185, 2972, 1780, 1669, 1480, 1367, 1269, 1082, 1026, 874.

LRMS (ESI+APCI) m/z $\left[\mathrm{C}_{11} \mathrm{H}_{20} \mathrm{NO}_{3}\right]^{+}\left([\mathrm{M}+\mathrm{H}]^{+}\right)$calculated 214.1, found 214.2.<smiles>CC/C=C/CCC(=O)NOCCO</smiles>

(E)-N-(pivaloyloxy)oct-4-enamide (1f)

(Prepared according to the general procedure A from ethyl (E)-4-octenoate synthesized according to a literature procedure. ${ }^{5}$ )

Yield: $57 \%$. White solid. $\mathbf{R}_{f}=0.70$ (hexane/EtOAc 1:1).

${ }^{1} \mathbf{H}$ NMR $\left(500 \mathrm{MHz} \mathrm{CDCl}_{3}\right) \delta 8.94(\mathrm{~s}, 1 \mathrm{H}), 5.56-5.48(\mathrm{~m}, 1 \mathrm{H}), 5.47-5.39(\mathrm{~m}, 1 \mathrm{H}), 2.42-2.29(\mathrm{~m}$, $4 \mathrm{H}), 2.02-1.94(\mathrm{~m}, 2 \mathrm{H}), 1.38(\mathrm{dt}, \mathrm{J}=14.7,7.4 \mathrm{~Hz}, 2 \mathrm{H}), 1.33(\mathrm{~s}, 9 \mathrm{H}), 0.89(\mathrm{t}, \mathrm{J}=7.4 \mathrm{~Hz}, 3 \mathrm{H})$.

${ }^{13} \mathrm{C}$ NMR $\left(126 \mathrm{MHz}, \mathrm{CDCl}_{3}\right) \delta 176.7,171.0,132.3,127.8,38.3,34.6,33.2,27.9,27.0,22.5,13.6$.

IR (neat, $\mathrm{cm}^{-1}$ ) v 3184, 2961, 1777, 1665, 1517, 1372, 1273, 1092, 974.

LRMS (ESI+APCI) m/z $\left[\mathrm{C}_{13} \mathrm{H}_{24} \mathrm{NO}_{3}\right]^{+}\left([\mathrm{M}+\mathrm{H}]^{+}\right)$calculated 242.2, found 242.2.<smiles>O=C(CC/C=C/c1ccccc1)NOCc1ccccc1</smiles>

(E)-5-phenyl-N-(pivaloyloxy)pent-4-enamide (1g)

(Prepared according to the general procedure B from (E)-5-phenylpent-4-enoic acid synthesized according to a literature procedure. ${ }^{6}$ )

Yield: $73 \%$. White solid. $\mathbf{R}_{f}=0.35$ (hexane/EtOAc 4:1).

${ }^{1} \mathbf{H}$ NMR (500 MHz, CDCl $\left.{ }_{3}\right) \delta 8.90(\mathrm{~s}, 1 \mathrm{H}), 7.39-7.35(\mathrm{~m}, 2 \mathrm{H}), 7.32(\mathrm{dd}, J=8.5,6.8 \mathrm{~Hz}, 2 \mathrm{H}), 7.26-$ $7.21(\mathrm{~m}, 1 \mathrm{H}), 6.48(\mathrm{~d}, J=15.8 \mathrm{~Hz}, 1 \mathrm{H}), 6.25(\mathrm{dt}, J=15.8,6.9 \mathrm{~Hz}, 1 \mathrm{H}), 2.65-2.58(\mathrm{~m}, 2 \mathrm{H}), 2.45(\mathrm{t}, J$ $=7.4 \mathrm{~Hz}, 2 \mathrm{H}), 1.34(\mathrm{~s}, 9 \mathrm{H})$.

${ }^{13} \mathrm{C}$ NMR $\left(126 \mathrm{MHz}, \mathrm{CDCl}_{3}\right) \delta 176.7,170.7,137.2,131.5,128.5,128.0,127.3,126.1,38.4,32.8$, 28.2, 27.0.

IR (neat, $\mathrm{cm}^{-1}$ ) v 3185, 2974, 2937, 1778, 1667, 1480, 1078, 965, 744, 692.

LRMS (ESI+APCI) m/z $\left[\mathrm{C}_{16} \mathrm{H}_{22} \mathrm{NO}_{3}\right]^{+}\left([\mathrm{M}+\mathrm{H}]^{+}\right)$calculated 276.2, found 276.2. 
$\sum_{(Z / E=17: 1)}^{1 h}$

(Z)-5-phenyl-N-(pivaloyloxy)pent-4-enamide (1h)

(Prepared according to the general procedure $\mathbf{B}$ from (Z)-5-phenylpent-4-enoic acid synthesized according to a literature procedure. ${ }^{6}$ )

Yield: $67 \%$. White solid. $\mathbf{R}_{\boldsymbol{f}}=0.35$ (hexane/EtOAc 4:1).

${ }^{1} \mathrm{H}$ NMR $\left(500 \mathrm{MHz}, \mathrm{CDCl}_{3}\right) \delta 9.01(\mathrm{~s}, 1 \mathrm{H}), 7.37-7.33(\mathrm{~m}, 2 \mathrm{H}), 7.30-7.23(\mathrm{~m}, 3 \mathrm{H}), 6.51(\mathrm{~d}, J=11.6$ $\mathrm{Hz}, 1 \mathrm{H}), 5.67(\mathrm{dt}, J=11.6,7.2 \mathrm{~Hz}, 1 \mathrm{H}), 2.77-2.70(\mathrm{~m}, 2 \mathrm{H}), 2.39(\mathrm{t}, J=7.5 \mathrm{~Hz}, 2 \mathrm{H}), 1.33(\mathrm{~s}, 9 \mathrm{H})$.

${ }^{13} \mathrm{C}$ NMR $\left(126 \mathrm{MHz} \mathrm{CDCl}_{3}\right) \delta 176.6,170.6,137.0,130.5,129.8,128.7,128.3,126.9,38.3,32.9$, 27.0, 23.9.

IR (neat, $\mathrm{cm}^{-1}$ ) v 3189, 2975, 2937, 1779, 1668, 1480, 1082, 1026, 768, 699.

LRMS (ESI+APCI) m/z [ $\left.\mathrm{C}_{16} \mathrm{H}_{22} \mathrm{NO}_{3}\right]^{+}\left([\mathrm{M}+\mathrm{H}]^{+}\right)$calculated 276.2 , found 276.1 .<smiles>C=C(C)CCC(=O)NOCCOC</smiles>

4-methyl-N-(pivaloyloxy)pent-4-enamide (1i)

(Prepared according to the general procedure $\mathbf{A}$ from ethyl 4-methyl-4-pentenoate purchased from Sigma Aldrich.)

Yield: $67 \%$. White solid. $\mathbf{R}_{\boldsymbol{f}}=0.80$ (hexane/EtOAc 1:1).

${ }^{1} \mathrm{H}$ NMR $\left(400 \mathrm{MHz}, \mathrm{CDCl}_{3}\right) \delta 9.53(\mathrm{~s}, 1 \mathrm{H}), 4.75(\mathrm{~s}, 1 \mathrm{H}), 4.71(\mathrm{~s}, 1 \mathrm{H}), 2.43-2.31(\mathrm{~m}, 4 \mathrm{H}), 1.73(\mathrm{~s}, 3 \mathrm{H})$, $1.29(\mathrm{~s}, 9 \mathrm{H})$.

${ }^{13} \mathrm{C}$ NMR $\left(100 \mathrm{MHz}, \mathrm{CDCl}_{3}\right) \delta 176.5,171.1,143.8,110.7,38.3,32.4,31.1,27.0,22.4$.

IR (neat, $\mathrm{cm}^{-1}$ ) v 3186, 2973, 1780, 1670, 1481, 1369, 1270, 1084, 1031, 887.

LRMS (ESI+APCI) m/z $\left[\mathrm{C}_{11} \mathrm{H}_{20} \mathrm{NO}_{3}\right]^{+}\left([\mathrm{M}+\mathrm{H}]^{+}\right)$calculated 214.1, found 214.2.<smiles>CONC(=O)C(C)C/C=C/O</smiles>

(E)-2-methyl-N-(pivaloyloxy)pent-4-enamide (1j- $\left.\mathbf{d}_{\mathbf{1}}\right)$

(Prepared according to the general procedure $\mathbf{B}$ )

Yield: $58 \%$. Colorless oil. $\mathbf{R}_{\boldsymbol{f}}=0.30$ (hexane/EtOAc4:1).

${ }^{1} \mathrm{H}$ NMR $\left(500 \mathrm{MHz}, \mathrm{CDCl}_{3}\right) \delta 9.43(\mathrm{~s}, 1 \mathrm{H}), 5.76(\mathrm{dt}, J=17.1,6.5 \mathrm{~Hz}, 1 \mathrm{H}), 5.07(\mathrm{~d}, J=16.9 \mathrm{~Hz}, 1 \mathrm{H})$, $2.46-2.37(\mathrm{~m}, 2 \mathrm{H}), 2.20-2.13(\mathrm{~m}, 2 \mathrm{H}), 1.29(\mathrm{~s}, 9 \mathrm{H}), 1.18(\mathrm{~d}, J=6.7 \mathrm{~Hz}, 3 \mathrm{H})$.

${ }^{13} \mathrm{C}$ NMR $\left(126 \mathrm{MHz}, \mathrm{CDCl}_{3}\right) \delta 176.5,174.3,135.0,117.0(\mathrm{t}, J=96.1 \mathrm{~Hz}), 38.3,37.9,37.7,27.0$, 17.0. 


\section{Synthesis of cationic $\operatorname{Ir}($ III) catalyst}

General Procedure:

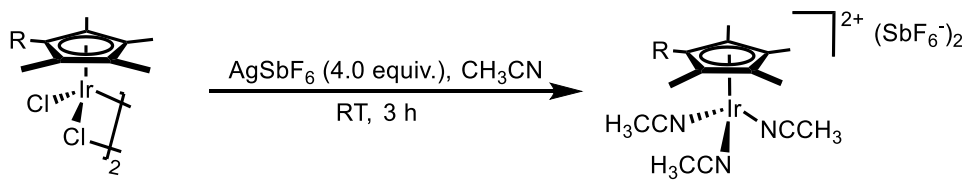

To a suspension of $\left[\mathrm{Cp}^{\mathrm{x}} \mathrm{IrCl}_{2}\right]_{2}$ (1.0 equiv.) in dry acetonitrile $(0.5 \mathrm{M})$ was added $\mathrm{AgSbF} \mathrm{F}_{6}$ (4.0 equiv.) in one portion. The mixture was stirred at room temperature for $3 \mathrm{~h}$. The reaction was filtered through a pad of celite ${ }^{\circledR}$ and the filtrate was concentrated by rotary evaporation. The resulting solid was dried under high vacuum and used without further purification.

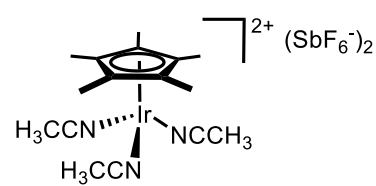

$\left[\mathrm{Cp} * \mathrm{Ir}\left(\mathrm{CH}_{3} \mathrm{CN}\right)_{3}\right]\left(\mathrm{SbF}_{6}\right)_{2}$

$\left(\left[\mathrm{Cp}^{*} \mid \mathrm{ICl}_{2}\right]_{2}\right.$ was synthesized according to a literature procedure. $\left.{ }^{1}\right)$

Yield: $87 \%$. Light yellow solid.

${ }^{1} \mathbf{H}$ NMR (500 MHz, CD 3 CN) $\delta 1.99(\mathrm{~s}, 8 \mathrm{H}), 1.78(\mathrm{~s}, 15 \mathrm{H})$.

${ }^{13} \mathrm{C}$ NMR $\left(126 \mathrm{MHz}, \mathrm{CD}_{3} \mathrm{CN}\right) \delta 124.5,94.6,9.5,2.1$.

IR (neat, $\mathrm{cm}^{-1}$ ) v 2949, 2329, 1457, 1391, 1032, 649, 471.

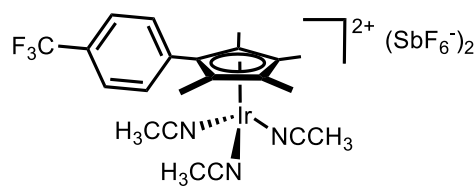

$\left[\mathrm{Cp}^{*{ }^{p C F 3}} \operatorname{Ir}\left(\mathrm{CH}_{3} \mathrm{CN}\right)_{3}\right]\left(\mathrm{SbF}_{6}\right)_{2}$

$\left(\left[\mathrm{Cp}^{*}{ }^{* \mathrm{CF} 3} \mid \mathrm{ICl}_{2}\right]_{2}\right.$ was synthesized according to a literature procedure. $\left.{ }^{1}\right)$

Yield: $80 \%$. Light yellow solid.

${ }^{1} \mathrm{H}$ NMR (500 MHz, CD $\left.{ }_{3} \mathrm{CN}\right) \delta 7.86(\mathrm{~d}, \mathrm{~J}=8.1 \mathrm{~Hz}, 2 \mathrm{H}), 7.68(\mathrm{~d}, \mathrm{~J}=8.1 \mathrm{~Hz}, 2 \mathrm{H}), 1.99(\mathrm{~s}, 5 \mathrm{H}), 1.89(\mathrm{~s}$, $6 \mathrm{H}), 1.75(\mathrm{~s}, 6 \mathrm{H})$.

${ }^{13} \mathrm{C}$ NMR (126 MHz, CD 3 CN) $\delta 131.2,131.1,131.1$ (q, J = 32.8 Hz), 126.2 (q, J = 3.8 Hz) 124.1 (q, J = $272 \mathrm{~Hz}), 123.6,103.4,91.3,84.9,9.2,8.2,3.1(\mathrm{p}, \mathrm{J}=84.5 \mathrm{~Hz})$.

${ }^{19} \mathrm{~F}$ NMR $\left(471 \mathrm{MHz}, \mathrm{CD}_{3} \mathrm{CN}\right) \delta-63.3$.

IR (neat, $\mathrm{cm}^{-1}$ ) v 2949, 2331, 1410, 1325, 1168, 1124, 1068, 1026, 858, 650. 


\section{Synthesis of $\boldsymbol{p}$-lactams}

\section{General Procedure:}

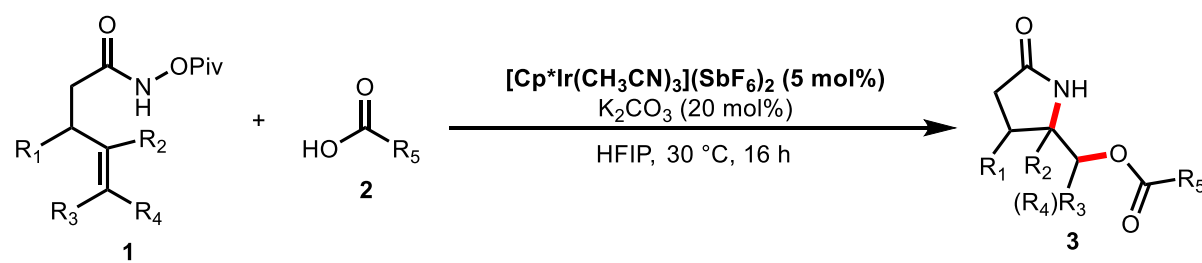

Alkenyl $\mathrm{N}$ - pivaloylhydroxamate $(0.10 \mathrm{mmol})$, carboxylic acid $\left(0.25 \mathrm{mmol}, 2.5\right.$ equiv.), $\mathrm{K}_{2} \mathrm{CO}_{3}(0.02$ $\mathrm{mmol}, 20 \mathrm{~mol} \%)$ and HFIP $(170 \mu \mathrm{L})$ were combined in a 1 dram vial with a magnetic stir bar. In a separate vial, $\left[\mathrm{Cp} * \mathrm{Ir}\left(\mathrm{CH}_{3} \mathrm{CN}\right)_{3}\right]\left(\mathrm{SbF}_{6}\right)_{2}(0.005 \mathrm{mmol}, 5.0 \mathrm{~mol} \%)$ was dissolved in HFIP $(170 \mu \mathrm{L})$, and then transferred to the first vial. The reaction is stirred at $30^{\circ} \mathrm{C}$. After 16 hours, $2 \mathrm{~mL}$ of $2 \mathrm{M}$ aqueous $\mathrm{Na}_{2} \mathrm{CO}_{3}$ was added and the mixture was extracted 4 times with $2 \mathrm{~mL} \mathrm{DCM}$. The organic layer was dried over anhydrous $\mathrm{Na}_{2} \mathrm{SO}_{4}$, solids were filtered, and the filtrate was concentrated by rotary evaporation. A crude ${ }^{1} \mathrm{H}$ NMR spectrum was collected with mesitylene as internal standard. The crude mixture was then purified by column chromatography.<smiles>CCC(=O)OCC1CCC(=O)N1</smiles>

(5-oxopyrrolidin-2-yl)methyl propionate (3aa)

Yield: $68 \%, 8.2: 1$ rr. White solid. $\mathbf{R}_{f}=0.60(\mathrm{MeOH} / \mathrm{DCM} 1: 10)$.

${ }^{1} \mathrm{H}$ NMR (400 MHz, CDCl $) \delta 6.43$ (brs, $\left.1 \mathrm{H}\right), 4.25-4.17(\mathrm{~m}, 1 \mathrm{H}), 3.96-3.88(\mathrm{~m}, 2 \mathrm{H}), 2.44-2.22$ $(\mathrm{m}, 5 \mathrm{H}), 1.90-1.79(\mathrm{~m}, 1 \mathrm{H}), 1.16(\mathrm{t}, \mathrm{J}=7.6 \mathrm{~Hz}, 3 \mathrm{H})$.

${ }^{13} \mathrm{C}$ NMR $\left(100 \mathrm{MHz}, \mathrm{CDCl}_{3}\right) \delta 178.0,174.1,66.9,52.8,29.5,27.4,23.3,9.0$.

IR (neat, $\mathrm{cm}^{-1}$ ) v 3223, 2944, 1734, 1690, 1462, 1271, 1181, 1021, 958, 648

LRMS (ESI+APCl) $\mathrm{m} / \mathrm{z}\left[\mathrm{C}_{8} \mathrm{H}_{14} \mathrm{NO}_{3}\right]^{+}\left([\mathrm{M}+\mathrm{H}]^{+}\right)$calculated 172.1, found 172.1 .

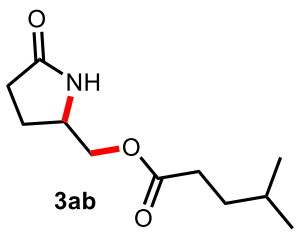

(5-oxopyrrolidin-2-yl)methyl 4-methylpentanoate (3ab)

Yield: $63 \%$, 8.4:1 rr. Colorless oil. $\mathbf{R}_{f}=0.70(\mathrm{MeOH} / \mathrm{DCM} 1: 10)$.

${ }^{1} \mathrm{H}$ NMR $\left(500 \mathrm{MHz}, \mathrm{CDCl}_{3}\right) \delta 5.98(\mathrm{brs}, 1 \mathrm{H}), 4.27-4.20(\mathrm{~m}, 1 \mathrm{H}), 3.96-3.87(\mathrm{~m}, 2 \mathrm{H}), 2.45-2.25$ $(\mathrm{m}, 5 \mathrm{H}), 1.90-1.81(\mathrm{~m}, 1 \mathrm{H}), 1.64-1.50(\mathrm{~m}, 3 \mathrm{H}), 0.93(\mathrm{~s}, 3 \mathrm{H}), 0.92(\mathrm{~s}, 3 \mathrm{H})$

${ }^{13} \mathrm{C}$ NMR $\left(126 \mathrm{MHz}, \mathrm{CDCl}_{3}\right) \delta 177.7,173.7,66.9,52.7,33.7,32.2,29.4,27.7,23.3,22.2$.

IR (neat, $\mathrm{cm}^{-1}$ ) v 2955, 1734, 1693, 1462, 1385, 1264, 1165, 1104, 646.

LRMS (ESI+APCI) m/z [ $\left.\mathrm{C}_{11} \mathrm{H}_{20} \mathrm{NO}_{3}\right]^{+}\left([\mathrm{M}+\mathrm{H}]^{+}\right)$calculated 214.1, found 214.2. 


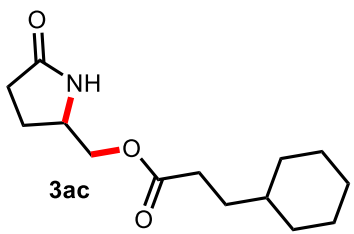

(5-oxopyrrolidin-2-yl)methyl 3-cyclohexylpropanoate (3ac)

$40{ }^{\circ} \mathrm{C}$ was applied.

Yield: $76 \%, 6.8: 1 \mathrm{rr}$. White solid. $\mathbf{R}_{f}=0.70(\mathrm{MeOH} / \mathrm{DCM} 1: 10)$.

${ }^{1} \mathrm{H}$ NMR $\left(500 \mathrm{MHz}, \mathrm{CDCl}_{3}\right) \delta 6.15$ (brs, $\left.1 \mathrm{H}\right), 4.26-4.19(\mathrm{~m}, 1 \mathrm{H}), 3.95-3.88(\mathrm{~m}, 2 \mathrm{H}), 2.44-2.24$ $(\mathrm{m}, 5 \mathrm{H}), 1.90-1.81(\mathrm{~m}, 1 \mathrm{H}), 1.75-1.63(\mathrm{~m}, 5 \mathrm{H}), 1.57-1.50(\mathrm{~m}, 2 \mathrm{H}), 1.29-1.11(\mathrm{~m}, 4 \mathrm{H}), 0.96-$ $0.86(\mathrm{~m}, 2 \mathrm{H})$.

${ }^{13} \mathrm{C}$ NMR $\left(126 \mathrm{MHz}, \mathrm{CDCl}_{3}\right) \delta 177.9,173.8,66.9,52.8,37.2,32.9,32.2,31.7,29.5,26.5,26.2,23.3$. IR (neat, $\left.\mathrm{cm}^{-1}\right)$ v 2920, 2849, 1735, 1694, 1448, 1383, 1274, 1160, 648.

LRMS (ESI+APCI) m/z $\left[\mathrm{C}_{14} \mathrm{H}_{24} \mathrm{NO}_{3}\right]^{+}\left([\mathrm{M}+\mathrm{H}]^{+}\right)$calculated 254.2, found 254.2.

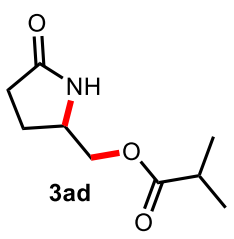

(5-oxopyrrolidin-2-yl)methyl isobutyrate (3ad)

$40{ }^{\circ} \mathrm{C}$ was applied.

Yield: $78 \%, 11: 1$ rr. Colorless oil. $\mathbf{R}_{f}=0.20$ (EA).

${ }^{1} \mathrm{H}$ NMR $\left(500 \mathrm{MHz}, \mathrm{CDCl}_{3}\right) \delta 6.14(\mathrm{~s}, 1 \mathrm{H}), 4.27-4.22(\mathrm{~m}, 1 \mathrm{H}), 3.96-3.88(\mathrm{~m}, 2 \mathrm{H}), 2.60$ (hept, $J=$ $7.0 \mathrm{~Hz}, 1 \mathrm{H}), 2.42-2.35(\mathrm{~m}, 2 \mathrm{H}), 2.35-2.25(\mathrm{~m}, 1 \mathrm{H}), 1.91-1.83(\mathrm{~m}, 1 \mathrm{H}), 1.20(\mathrm{~s}, 3 \mathrm{H}), 1.19(\mathrm{~s}, 3 \mathrm{H})$.

${ }^{13} \mathrm{C}$ NMR $\left(126 \mathrm{MHz}, \mathrm{CDCl}_{3}\right) \delta 177.9,176.8,66.8,52.8,33.9,29.5,23.3,18.9$.

IR (neat, $\mathrm{cm}^{-1}$ ) v 3228, 2973, 2938, 1731, 1693, 1192, 1153.

LRMS (ESI+APCI) $\mathrm{m} / \mathrm{z}\left[\mathrm{C}_{9} \mathrm{H}_{16} \mathrm{NO}_{3}\right]^{+}\left([\mathrm{M}+\mathrm{H}]^{+}\right)$calculated 186.1, found 186.1 .

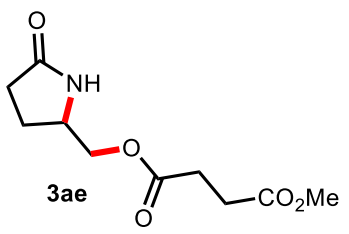

methyl ((5-oxopyrrolidin-2-yl)methyl) succinate (3ae)

1.0 equivalent of $\mathrm{K}_{2} \mathrm{CO}_{3}$ was used.

Yield: $58 \%, 9.4: 1 \mathrm{rr}$. White solid. $\mathbf{R}_{f}=0.50(\mathrm{MeOH} / \mathrm{DCM} 1: 10)$.

${ }^{1} \mathrm{H}$ NMR $\left(500 \mathrm{MHz}, \mathrm{CDCl}_{3}\right) \delta 6.45$ (brs, $\left.1 \mathrm{H}\right), 4.25-4.18(\mathrm{~m}, 1 \mathrm{H}), 3.99-3.90(\mathrm{~m}, 2 \mathrm{H}), 3.71(\mathrm{~s}, 3 \mathrm{H})$, $2.70-2.62(\mathrm{~m}, 4 \mathrm{H}), 2.44-2.22(\mathrm{~m}, 3 \mathrm{H}), 1.86-1.77(\mathrm{~m}, 1 \mathrm{H})$.

${ }^{13} \mathrm{C}$ NMR $\left(126 \mathrm{MHz}, \mathrm{CDCl}_{3}\right) \delta 177.9,172.8,172.0,67.3,52.7,52.0,29.5,29.0,28.9,23.1$.

IR $\left(\right.$ neat, $\mathrm{cm}^{-1}$ ) v 2950, 1730, 1690, 1437, 1363, 1157, 1001, 846, 648.

LRMS (ESI+APCI) m/z [ $\left.\mathrm{C}_{10} \mathrm{H}_{16} \mathrm{NO}_{5}\right]^{+}\left([\mathrm{M}+\mathrm{H}]^{+}\right)$calculated 230.1, found 230.2 . 


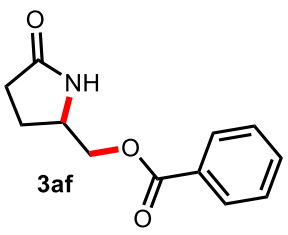

(5-oxopyrrolidin-2-yl)methyl benzoate (3af)

1.0 equivalent of $\mathrm{K}_{2} \mathrm{CO}_{3}$ was used.

Yield: 59\%, 8.5:1 rr. White solid. $\mathbf{R}_{f}=0.70(\mathrm{MeOH} / \mathrm{DCM} \mathrm{1:10)}$.

${ }^{1}$ H NMR $\left(500 \mathrm{MHz}, \mathrm{CDCl}_{3}\right) \delta 8.08-8.03(\mathrm{~m}, 2 \mathrm{H}), 7.63-7.58(\mathrm{~m}, 1 \mathrm{H}), 7.50-7.45(\mathrm{~m}, 2 \mathrm{H}), 6.21$ (brs, $1 \mathrm{H}), 4.48(\mathrm{dd}, \mathrm{J}=11.3,3.8 \mathrm{~Hz}, 1 \mathrm{H}), 4.19(\mathrm{dd}, \mathrm{J}=11.2,7.1 \mathrm{~Hz}, 1 \mathrm{H}), 4.11-4.05(\mathrm{~m}, 1 \mathrm{H}), 2.50-$ $2.32(\mathrm{~m}, 3 \mathrm{H}), 2.01-1.93(\mathrm{~m}, 1 \mathrm{H})$.

${ }^{13} \mathrm{C}$ NMR $\left(126 \mathrm{MHz}, \mathrm{CDCl}_{3}\right) \delta 177.8,166.3,133.4,129.7,129.4,128.5,67.5,52.9,29.5,23.4$.

IR (neat, $\mathrm{cm}^{-1}$ ) v 3195, 2926, 1689, 1450, 1384, 1270, 1127, 796, 709.

LRMS (ESI+APCI) m/z [ $\left.\mathrm{C}_{12} \mathrm{H}_{14} \mathrm{NO}_{3}\right]^{+}\left([\mathrm{M}+\mathrm{H}]^{+}\right)$calculated 220.1, found 220.1 .

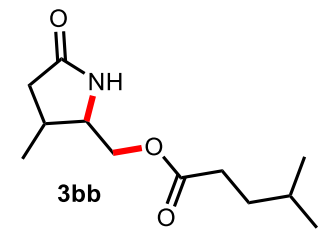

3-methyl-5-oxopyrrolidin-2-yl)methyl 4-methylpentanoate (3bb)

Yield: 71\%, >20:1 rr, 1.5:1 dr. Colorless oil. $\mathbf{R}_{f}=0.70(\mathrm{MeOH} / \mathrm{DCM} 1: 10)$.

${ }^{1} \mathrm{H}$ NMR $\left(500 \mathrm{MHz}, \mathrm{CDCl}_{3}\right) \delta 6.22 \& 6.20(\mathrm{brs}, 1 \mathrm{H}), 4.28 \& 4.26(\mathrm{t}, \mathrm{J}=4.1 \mathrm{~Hz}, 1 \mathrm{H}), 3.97 \& 3.90(\mathrm{dd}, \mathrm{J}$ $=11.4,7.3 \mathrm{~Hz}, 1 \mathrm{H}), 3.86-3.78 \& 3.49-3.42(\mathrm{~m}, 1 \mathrm{H}), 2.79-2.66 \& 2.29-2.16(\mathrm{~m}, 1 \mathrm{H}), 2.56 \&$ $2.45(\mathrm{dd}, \mathrm{J}=16.7,8.6 \mathrm{~Hz}, 1 \mathrm{H}), 2.38-2.32(\mathrm{~m}, 2 \mathrm{H}), 2.08$ \& $2.03(\mathrm{dd}, \mathrm{J}=16.9,8.9 \mathrm{~Hz}, 1 \mathrm{H}), 1.62-$ $1.50(\mathrm{~m}, 3 \mathrm{H}), 1.19 \& 1.12(\mathrm{~d}, \mathrm{~J}=7.1 \mathrm{~Hz}, 3 \mathrm{H}), 0.92(\mathrm{~s}, 3 \mathrm{H}), 0.91(\mathrm{~s}, 3 \mathrm{H})$.

${ }^{13} \mathrm{C}$ NMR $\left(126 \mathrm{MHz}, \mathrm{CDCl}_{3}\right) \delta 177.5,177.1,173.8,173.7,65.9,64.1,60.3,55.5,38.2,38.0,33.6$, 32.2, 32.2, 32.0, 31.5, 27.7, 22.2, 19.2, 14.7 .

IR $\left(\right.$ neat, $\left.\mathrm{cm}^{-1}\right)$ v 2956, 2870, 1734, 1695, 1456, 1375, 1263, 1169, 1105, 774.

LRMS (ESI+APCI) m/z [ $\left.\mathrm{C}_{12} \mathrm{H}_{22} \mathrm{NO}_{3}\right]^{+}\left([\mathrm{M}+\mathrm{H}]^{+}\right)$calculated 228.2, found 228.2.

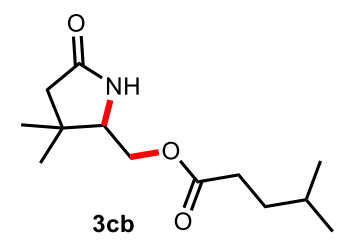

(3,3-dimethyl-5-oxopyrrolidin-2-yl)methyl 4-methylpentanoate (3cb) $40{ }^{\circ} \mathrm{C}$ was applied.

Yield: $93 \%,>20: 1 \mathrm{rr}$. White solid. $\mathbf{R}_{f}=0.80(\mathrm{MeOH} / \mathrm{DCM} 1: 10)$.

${ }^{1} \mathrm{H}$ NMR $\left(500 \mathrm{MHz}, \mathrm{CDCl}_{3}\right) \delta 6.22(\mathrm{brs}, 1 \mathrm{H}$ ), 4.27 (dd, J = 11.3, 3.6 Hz, 1H), 3.94 (dd, J = 11.3, $8.2 \mathrm{~Hz}$, $1 \mathrm{H}), 3.47(\mathrm{dd}, \mathrm{J}=8.2,3.6 \mathrm{~Hz}, 1 \mathrm{H}), 2.38-2.32(\mathrm{~m}, 2 \mathrm{H}), 2.25-2.14(\mathrm{~m}, 2 \mathrm{H}), 1.62-1.49(\mathrm{~m}, 3 \mathrm{H})$, $1.24(\mathrm{~s}, 3 \mathrm{H}), 1.11(\mathrm{~s}, 3 \mathrm{H}), 0.92(\mathrm{~s}, 3 \mathrm{H}), 0.91(\mathrm{~s}, 3 \mathrm{H})$.

${ }^{13} \mathrm{C} \mathrm{NMR}\left(126 \mathrm{MHz}, \mathrm{CDCl}_{3}\right) \delta 176.8,173.8,64.2,61.8,45.6,37.6,33.6,32.2,28.3,27.7,22.7,22.2$. IR $\left(\right.$ neat, $\left.\mathrm{cm}^{-1}\right)$ v 3216, 2956, 1736, 1695, 1466, 1368, 1267, 1168, 1105, 995, 683. 
<smiles>CC(=O)OC1CCC(=O)N1</smiles>

1-(5-oxopyrrolidin-2-yl)ethyl acetate (3dg)

From E- alkenyl N-pivaloylhydroxamate; $40^{\circ} \mathrm{C}$ was applied.

Yield: 77\%, >20:1 rr, >20:1 dr. White solid. $\mathbf{R}_{f}=0.65(\mathrm{MeOH} / \mathrm{DCM} 1: 10)$.

${ }^{1} \mathrm{H}$ NMR $\left(500 \mathrm{MHz}, \mathrm{CDCl}_{3}\right) \delta 6.06$ (brs, $\left.1 \mathrm{H}\right), 5.00(\mathrm{qd}, \mathrm{J}=6.4,3.8 \mathrm{~Hz}, 1 \mathrm{H}), 3.79(\mathrm{dt}, \mathrm{J}=8.5,4.5 \mathrm{~Hz}$, $1 \mathrm{H}), 2.43-2.30(\mathrm{~m}, 2 \mathrm{H}), 2.27-2.18(\mathrm{~m}, 1 \mathrm{H}), 2.08(\mathrm{~s}, 3 \mathrm{H}), 2.00-1.91(\mathrm{~m}, 1 \mathrm{H}), 1.24(\mathrm{~d}, \mathrm{~J}=6.4 \mathrm{~Hz}$, $3 \mathrm{H})$.

${ }^{13} \mathrm{C} \mathrm{NMR}\left(126 \mathrm{MHz}, \mathrm{CDCl}_{3}\right) \delta 178.2,170.4,71.3,57.2,29.5,21.9,21.2,15.0$.

IR (neat, $\mathrm{cm}^{-1}$ ) v 2938, 1734, 1696, 1371, 1240, 1072, 1021.

LRMS $(E S I+A P C I) ~ m / z ~\left[C_{8} \mathrm{H}_{14} \mathrm{NO}_{3}\right]^{+}\left([\mathrm{M}+\mathrm{H}]^{+}\right)$calculated 172.1, found 172.1.

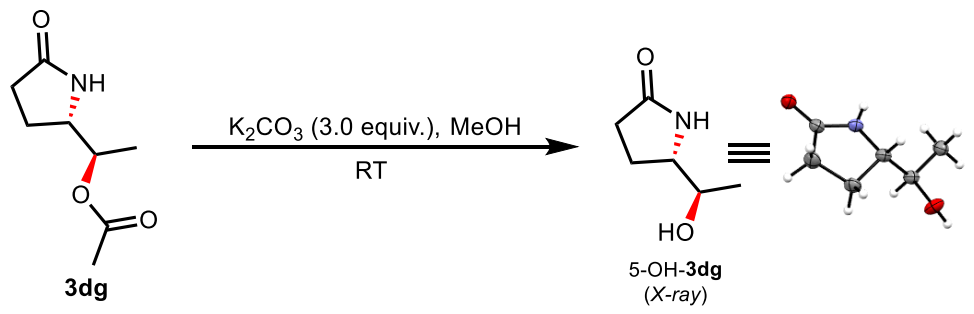

To a stirred solution of $3 \mathrm{dg}(24 \mathrm{mg}, 0.14 \mathrm{mmol})$ in $\mathrm{MeOH}(1 \mathrm{~mL})$ at room temperature was added $\mathrm{K}_{2} \mathrm{CO}_{3}(58 \mathrm{mg}, 0.42 \mathrm{mmol}$ ) in one portion. After $5 \mathrm{~h}$, solvent was removed by rotary evaporation. The residue was dissolved in DCM $(40 \mathrm{~mL})$, washed with saturated aqueous solution of $\mathrm{NH}_{4} \mathrm{Cl}$, and dried over anhydrous $\mathrm{Na}_{2} \mathrm{SO}_{4}$. The solids were filtered, and the filtrate was concentrated by rotary evaporation. The crude mixture was then purified by column chromatography, which gave $15.5 \mathrm{mg}$ of $5-\mathrm{OH}-3 \mathrm{dg}$. The relative stereochemistry was confirmed by X-ray crystallography.

Yield: $86 \%$. White solid. $\mathbf{R}_{f}=0.25(\mathrm{MeOH} / \mathrm{DCM} 1: 10)$.

${ }^{1} \mathrm{H}$ NMR $\left(500 \mathrm{MHz}, \mathrm{CDCl}_{3}\right.$ ) $\delta 7.12$ (brs, $1 \mathrm{H}$ ), 3.88 (qd, J = 6.4, 3.0 Hz, $1 \mathrm{H}$ ), 3.67 (ddd, J = 8.2, 5.1, 3.0 $\mathrm{Hz}, 1 \mathrm{H}), 3.48(\mathrm{brs}, 1 \mathrm{H}), 2.44-2.27(\mathrm{~m}, 2 \mathrm{H}), 2.14-2.00(\mathrm{~m}, 2 \mathrm{H}), 1.17(\mathrm{~d}, \mathrm{~J}=6.5 \mathrm{~Hz}, 3 \mathrm{H})$.

${ }^{13} \mathrm{C}$ NMR $\left(126 \mathrm{MHz}, \mathrm{CDCl}_{3}\right) \delta 179.7,68.5,59.9,30.3,20.4,18.0$.

IR (neat, $\mathrm{cm}^{-1}$ ) v 3256, 2971, 1666, 1460, 1422, 1377, 1268, 1074, 657.

LRMS (ESI+APCI) $\mathrm{m} / \mathrm{z}\left[\mathrm{C}_{6} \mathrm{H}_{12} \mathrm{NO}_{2}\right]^{+}\left([\mathrm{M}+\mathrm{H}]^{+}\right)$calculated 130.1, found 130.0.<smiles>CC(=O)OC(C)C1CCC(=O)N1</smiles>

1-(5-oxopyrrolidin-2-yl)ethyl acetate (3eg)

From Z- alkenyl N-pivaloylhydroxamate; $40{ }^{\circ} \mathrm{C}$ was applied. 
Yield: 75\%, >20:1 rr, >20:1 dr. White solid. $\mathbf{R}_{f}=0.60(\mathrm{MeOH} / \mathrm{DCM} 1: 10)$.

${ }^{1} \mathrm{H}$ NMR $\left(500 \mathrm{MHz}, \mathrm{CDCl}_{3}\right) \delta 6.69$ (brs, $\left.1 \mathrm{H}\right), 4.74(\mathrm{dq}, \mathrm{J}=7.5,6.3 \mathrm{~Hz}, 1 \mathrm{H}), 3.74-3.66(\mathrm{~m}, 1 \mathrm{H}), 2.42$ $-2.31(\mathrm{~m}, 2 \mathrm{H}), 2.29-2.21(\mathrm{~m}, 1 \mathrm{H}), 2.10(\mathrm{~s}, 3 \mathrm{H}), 1.84-1.76(\mathrm{~m}, 1 \mathrm{H}), 1.24(\mathrm{~d}, \mathrm{~J}=6.3 \mathrm{~Hz}, 3 \mathrm{H})$.

${ }^{13} \mathrm{C} \mathrm{NMR}\left(126 \mathrm{MHz}, \mathrm{CDCl}_{3}\right) \delta 178.0,170.4,73.7,57.6,30.0,23.5,21.2,16.2$.

IR (neat, $\mathrm{cm}^{-1}$ ) v 3184, 2984, 1735, 1686, 1378, 1235, 1062, 1022, 937, 801, 651.

LRMS (ESI+APCI) $\mathrm{m} / \mathrm{z}\left[\mathrm{C}_{8} \mathrm{H}_{14} \mathrm{NO}_{3}\right]^{+}\left([\mathrm{M}+\mathrm{H}]^{+}\right)$calculated 172.1, found 172.1.<smiles>CCCC(OC(C)=O)C1CCC(=O)N1</smiles>

1-(5-oxopyrrolidin-2-yl)butyl acetate (3fg)

From E- alkenyl N- pivaloylhydroxamate; $40{ }^{\circ} \mathrm{C}$ was applied.

Yield: 60\%, >20:1 rr, >20:1 dr. White solid. $\mathbf{R}_{f}=0.75(\mathrm{MeOH} / \mathrm{DCM} 1: 10)$.

${ }^{1} \mathrm{H}$ NMR $\left(500 \mathrm{MHz}, \mathrm{CDCl}_{3}\right) \delta 5.95$ (brs, $1 \mathrm{H}$ ), $5.02(\mathrm{dt}, \mathrm{J}=9.5,3.6 \mathrm{~Hz}, 1 \mathrm{H}$ ), 3.79 (ddd, J = 8.9, 5.1, 3.7 $\mathrm{Hz}, 1 \mathrm{H}), 2.41-2.27(\mathrm{~m}, 2 \mathrm{H}), 2.21-2.13(\mathrm{~m}, 1 \mathrm{H}), 2.09(\mathrm{~s}, 3 \mathrm{H}), 2.04-1.96(\mathrm{~m}, 1 \mathrm{H}), 1.63-1.55(\mathrm{~m}$, $1 \mathrm{H}), 1.50-1.37(\mathrm{~m}, 2 \mathrm{H}), 1.36-1.28(\mathrm{~m}, 1 \mathrm{H}), 0.94(\mathrm{t}, \mathrm{J}=7.2 \mathrm{~Hz}, 3 \mathrm{H})$.

${ }^{13} \mathrm{C} \mathrm{NMR}\left(126 \mathrm{MHz}, \mathrm{CDCl}_{3}\right) \delta 178.0,170.9,73.8,56.7,31.9,29.6,21.6,21.0,18.8,13.8$.

IR (neat, $\left.\mathrm{cm}^{-1}\right)$ v 3222, 2958, 1735, 1691, 1462, 1372, 1232, 1022.

LRMS (ESI+APCI) m/z $\left[\mathrm{C}_{10} \mathrm{H}_{18} \mathrm{NO}_{3}\right]^{+}\left([\mathrm{M}+\mathrm{H}]^{+}\right)$calculated 200.1, found 200.2.

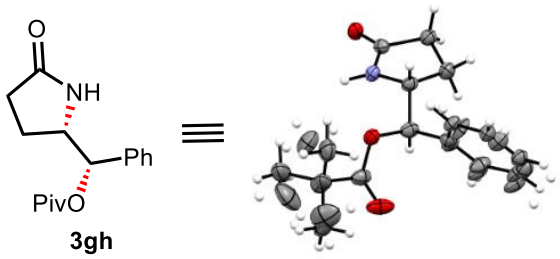

(5-oxopyrrolidin-2-yl)(phenyl)methyl pivalate (3gh)

From E- alkenyl N- pivaloylhydroxamate; $40{ }^{\circ} \mathrm{C}$ was applied.

Yield: $81 \%,>20: 1 \mathrm{rr},>20: 1 \mathrm{dr}$. White solid. $\mathbf{R}_{f}=0.65$ (EA).

${ }^{1} \mathrm{H}$ NMR $\left(500 \mathrm{MHz}, \mathrm{CDCl}_{3}\right) \delta 7.42-7.31(\mathrm{~m}, 5 \mathrm{H}), 5.80(\mathrm{~s}, 1 \mathrm{H}), 5.54(\mathrm{~d}, J=6.9 \mathrm{~Hz}, 1 \mathrm{H}), 4.02(\mathrm{td}, J=$ 7.5, $5.3 \mathrm{~Hz}, 1 \mathrm{H}), 2.41-2.26(\mathrm{~m}, 2 \mathrm{H}), 2.11-2.00(\mathrm{~m}, 1 \mathrm{H}), 1.92-1.82(\mathrm{~m}, 1 \mathrm{H}), 1.24(\mathrm{~s}, 9 \mathrm{H})$.

${ }^{13} \mathrm{C} \mathrm{NMR}\left(126 \mathrm{MHz}, \mathrm{CDCl}_{3}\right) \delta 177.7,177.1,136.9,128.8,128.7,126.5,78.4,57.9,38.9,29.6,27.1$, 23.3.

IR (neat, $\mathrm{cm}^{-1}$ ) v 3209, 2971, 2933, 2872, 1728, 1696, 1277, 1144, 702.

LRMS (ESI+APCI) $\mathrm{m} / \mathrm{z}\left[\mathrm{C}_{16} \mathrm{H}_{22} \mathrm{NO}_{3}\right]^{+}\left([\mathrm{M}+\mathrm{H}]^{+}\right)$calculated 276.1, found 276.2.

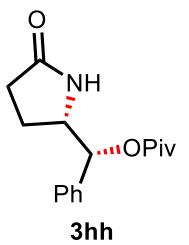

(5-oxopyrrolidin-2-yl)(phenyl)methyl pivalate (3hh)

From Z- alkenyl N-pivaloylhydroxamate; $40{ }^{\circ} \mathrm{C}$ was applied. 
Yield: $41 \%,>20: 1 \mathrm{rr}, 1.8: 1 \mathrm{dr}$. colorless oil. $\mathbf{R}_{f}=0.65(\mathrm{EA})$.

${ }^{1} \mathrm{H}$ NMR $\left(500 \mathrm{MHz}, \mathrm{CDCl}_{3}\right) \delta 7.43-7.30(\mathrm{~m}, 5 \mathrm{H}), 5.86 \& 5.65(\mathrm{~s}, 1 \mathrm{H}), 5.72 \& 5.53(\mathrm{~d}, J=5.8 \mathrm{~Hz}, \& \mathrm{~J}$ $=6.9 \mathrm{~Hz}, 1 \mathrm{H}), 4.06-4.00(\mathrm{~m}, 1 \mathrm{H}), 2.37-1.83(\mathrm{~m}, 4 \mathrm{H}), 1.25 \& 1.24(\mathrm{~s}, 9 \mathrm{H})$.

${ }^{13} \mathrm{C}$ NMR $\left(126 \mathrm{MHz}, \mathrm{CDCl}_{3}\right) \delta 177.9,177.8,177.3,177.1,136.9,136.5,128.8,128.8,128.7,128.6$, 126.7, 126.6, 78.4, 76.3, 57.9, 57.8, 38.9, 38.9, 29.6, 29.2, 27.1, 27.1, 23.3, 22.6.

IR $\left(\right.$ neat, $\left.\mathrm{cm}^{-1}\right)$ v 3211, 2971, 2934, 2873, 1729, 1693, 1279, 1144, 702.

LRMS (ESI+APCI) $\mathrm{m} / \mathrm{z}\left[\mathrm{C}_{16} \mathrm{H}_{22} \mathrm{NO}_{3}\right]^{+}\left([\mathrm{M}+\mathrm{H}]^{+}\right)$calculated 276.1, found 276.1 .

\section{General procedure with halide as nucleophile:}

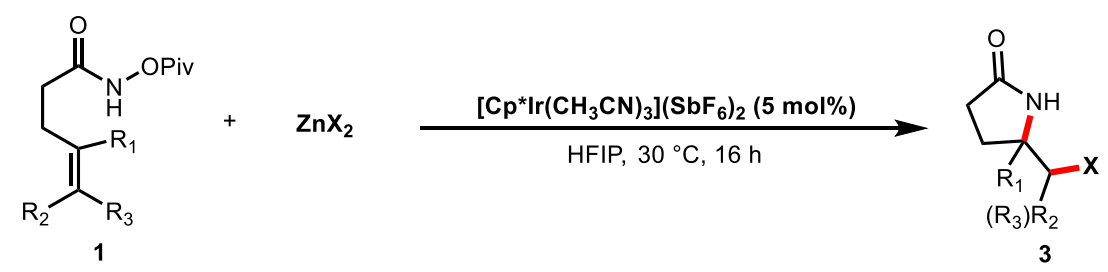

Alkenyl $\mathrm{N}$ - pivaloylhydroxamate $(0.10 \mathrm{mmol}), \mathrm{ZnX} 2(0.25 \mathrm{mmol}, 2.5$ equiv.) and $\mathrm{HFIP}(170 \mu \mathrm{L})$ were combined in a $1 \mathrm{dram}$ vial with a magnetic stir bar. In a separate vial, $\left[\mathrm{Cp} * \operatorname{Ir}\left(\mathrm{CH}_{3} \mathrm{CN}\right)_{3}\right]\left(\mathrm{SbF}_{6}\right)_{2}$ ( $0.005 \mathrm{mmol}, 5.0 \mathrm{~mol} \%)$ was dissolved in HFIP $(170 \mu \mathrm{L})$, and then transferred to the first vial. The reaction was stirred at $30{ }^{\circ} \mathrm{C}$. After 16 hours, $2 \mathrm{~mL}$ of $2 \mathrm{M}$ aqueous $\mathrm{Na}_{2} \mathrm{CO}_{3}$ was added and the mixture was extracted 4 times with $2 \mathrm{~mL}$ DCM. The combined organic layer was dried over anhydrous $\mathrm{Na}_{2} \mathrm{SO}_{4}$, the solids were filtered, and the filtrate was concentrated by rotary evaporation. A crude ${ }^{1} \mathrm{H}$ NMR spectrum was collected with mesitylene as internal standard. The crude mixture was then purified by column chromatography.

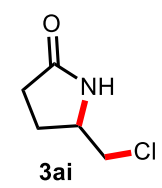

5-(chloromethyl)pyrrolidin-2-one (3ai)

Yield: $64 \%,>20: 1$ rr. White solid. $\mathbf{R}_{f}=0.65(\mathrm{MeOH} / \mathrm{DCM} \mathrm{1:10)}$.

${ }^{1} \mathrm{H}$ NMR $\left(500 \mathrm{MHz}, \mathrm{CDCl}_{3}\right) \delta 6.44$ (brs, $\left.1 \mathrm{H}\right), 4.00-3.94(\mathrm{~m}, 1 \mathrm{H}), 3.59$ (dd, J = 11.1, $4.7 \mathrm{~Hz}, 1 \mathrm{H}$ ), $3.49(\mathrm{dd}, \mathrm{J}=11.1,7.2 \mathrm{~Hz}, 1 \mathrm{H}), 2.50-2.30(\mathrm{~m}, 3 \mathrm{H}), 1.95-1.86(\mathrm{~m}, 1 \mathrm{H})$.

${ }^{13} \mathrm{C} \mathrm{NMR}\left(126 \mathrm{MHz}, \mathrm{CDCl}_{3}\right) \delta 177.8,55.2,48.1,29.7,24.7$.

IR (neat, $\mathrm{cm}^{-1}$ ) v 3178, 2955, 1671, 1462, 1387, 1284, 1084, 776, 646.

LRMS (ESI+APCI) $\mathrm{m} / \mathrm{z}\left[\mathrm{C}_{5} \mathrm{H}_{9} \mathrm{CINO}\right]^{+}\left([\mathrm{M}+\mathrm{H}]^{+}\right)$calculated 134.0, found 134.0.

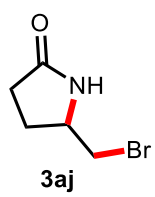

5-(bromomethyl)pyrrolidin-2-one (3aj)

Yield: 95\%, >20:1 rr. White solid. $\mathbf{R}_{f}=0.70(\mathrm{MeOH} / \mathrm{DCM} 1: 10)$.

${ }^{1}$ H NMR (500 MHz, $\left.\mathrm{CDCl}_{3}\right) \delta 6.75$ (brs, $\left.1 \mathrm{H}\right), 4.04-3.95(\mathrm{~m}, 1 \mathrm{H}), 3.44$ (dd, J = 10.3, $5.0 \mathrm{~Hz}, 1 \mathrm{H}$ ), $3.37(\mathrm{dd}, \mathrm{J}=10.3,6.9 \mathrm{~Hz}, 1 \mathrm{H}), 2.51-2.31(\mathrm{~m}, 3 \mathrm{H}), 1.95-1.86(\mathrm{~m}, 1 \mathrm{H})$.

${ }^{13} \mathrm{C}$ NMR $\left(126 \mathrm{MHz}, \mathrm{CDCl}_{3}\right) \delta 177.9,55.0,36.7,30.0,25.8$. 
IR (neat, $\mathrm{cm}^{-1}$ ) v 3173, 3096, 2917, 1669, 1461, 1328, 1281, 1072, 778, 499.

LRMS (ESI+APCI) $\mathrm{m} / \mathrm{z}\left[\mathrm{C}_{5} \mathrm{H}_{9} \mathrm{BrNO}\right]^{+}\left([\mathrm{M}+\mathrm{H}]^{+}\right)$calculated 178.0, found 178.0, 180.0.<smiles>CC1(CBr)CCC(=O)N1</smiles>

$3 \mathrm{ij}$

5-(bromomethyl)-5-methylpyrrolidin-2-one (3ij)

$40{ }^{\circ} \mathrm{C}$ was applied.

Yield: 63\%, >20:1 rr. Light yellow solid. $\mathbf{R}_{f}=0.75(\mathrm{MeOH} / \mathrm{DCM} 1: 10)$.

${ }^{1} \mathrm{H}$ NMR $\left(500 \mathrm{MHz}, \mathrm{CDCl}_{3}\right) \delta 7.04(\mathrm{~s}, 1 \mathrm{H}), 3.42(\mathrm{~s}, 2 \mathrm{H}), 2.55-2.38(\mathrm{~m}, 2 \mathrm{H}), 2.16$ (ddd, J = 13.3, 9.8, $5.8 \mathrm{~Hz}, 1 \mathrm{H}$ ), 2.00 (ddd, J = 13.3, 9.8, 7.4 Hz, 1H), 1.44 (s, 3H).

${ }^{13} \mathrm{C} \mathrm{NMR}\left(126 \mathrm{MHz}, \mathrm{CDCl}_{3}\right) \delta 177.2,59.2,42.6,32.2,30.6,26.2$.

IR (neat, $\mathrm{cm}^{-1}$ ) v 3221, 2970, 1689, 1416, 1379, 1193, 1103, 639, 508.

LRMS (ESI+APCI) $\mathrm{m} / \mathrm{z}\left[\mathrm{C}_{6} \mathrm{H}_{11} \mathrm{BrNO}\right]^{+}\left([\mathrm{M}+\mathrm{H}]^{+}\right)$calculated 192.0, found 192.0, 194.0.

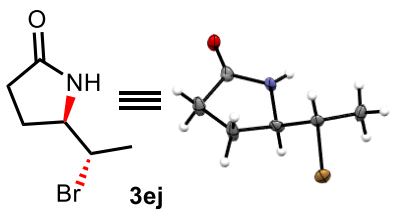

(R)-5-((S)-1-bromoethyl)pyrrolidin-2-one (3ej)

From Z- alkenyl N- pivaloylhydroxamate; $4{ }^{\circ} \mathrm{C}$ was applied.

Yield: 70\%, >20:1 rr, 8.3:1 dr. white solid. $\mathbf{R}_{f}=0.65(\mathrm{MeOH} / \mathrm{DCM} 1: 10)$.

${ }^{1} \mathrm{H}$ NMR $\left(400 \mathrm{MHz}, \mathrm{CDCl}_{3}\right) \delta 7.00(\mathrm{~s}, 1 \mathrm{H}), 4.15-4.03(\mathrm{~m}, 1 \mathrm{H}), 3.86(\mathrm{dt}, \mathrm{J}=8.0,5.2 \mathrm{~Hz}, 1 \mathrm{H}), 2.50-$ $2.41(\mathrm{~m}, 1 \mathrm{H}), 2.41-2.35(\mathrm{~m}, 1 \mathrm{H}), 2.35-2.27(\mathrm{~m}, 1 \mathrm{H}), 2.06-1.94(\mathrm{~m}, 1 \mathrm{H}), 1.71(\mathrm{~d}, \mathrm{~J}=6.7 \mathrm{~Hz}, 3 \mathrm{H})$.

${ }^{13} \mathrm{C}$ NMR $\left(101 \mathrm{MHz}, \mathrm{CDCl}_{3}\right) \delta 178.5,60.2,52.9,30.0,24.5,21.4$.

IR (neat, $\mathrm{cm}^{-1}$ ) v 3218, 2971, 2925, 1692, 1457, 1380, 1269, 756, 618.

HRMS (ASAP) $\mathrm{m} / \mathrm{z}[\mathrm{C} 6 \mathrm{H} 11 \mathrm{BrNO}]^{+}\left([\mathrm{M}+\mathrm{H}]^{+}\right)$calculated 192.0024, 193.9998, found 192.0030, 194.0010 .

\section{Synthesis of $\delta$-lactams}

\section{General Procedure:}<smiles>C=CCC(Br)C(=O)NOCCO</smiles>

1<smiles>[R]C(=O)O</smiles>

2

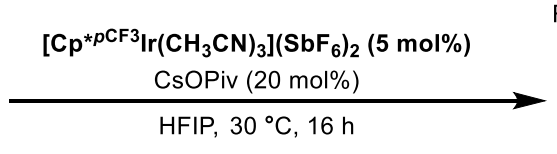

HFIP, $30^{\circ} \mathrm{C}, 16 \mathrm{~h}$

Alkenyl $\mathrm{N}$ - pivaloylhydroxamate $(0.10 \mathrm{mmol})$, carboxylic acid $(0.25 \mathrm{mmol}, 2.5$ equiv.), CsOPiv (0.02 $\mathrm{mmol}, 20 \mathrm{~mol} \%)$ and HFIP $(170 \mu \mathrm{L})$ were combined in a 1 dram vial with a magnetic stir bar. In a separate vial, $\left[\mathrm{Cp}^{*{ }^{p C F 3}} \mathrm{Ir}\left(\mathrm{CH}_{3} \mathrm{CN}\right)_{3}\right]\left(\mathrm{SbF}_{6}\right)_{2}(0.005 \mathrm{mmol}, 5.0 \mathrm{~mol} \%)$ was dissolved in HFIP $(170 \mu \mathrm{L})$, and then transferred to the first vial. The reaction was stirred at $30{ }^{\circ} \mathrm{C}$. After 16 hours, $2 \mathrm{~mL}$ of 2 $\mathrm{M}$ aqueous $\mathrm{Na}_{2} \mathrm{CO}_{3}$ was added and the mixture was extracted 4 times with $2 \mathrm{~mL}$ DCM. The 
combined organic layer was dried over anhydrous $\mathrm{Na}_{2} \mathrm{SO}_{4}$, solids were filtered, and the filtrate was concentrated by rotary evaporation. $A$ crude ${ }^{1} \mathrm{H}$ NMR spectrum was collected with mesitylene as internal standard. The crude mixture was then purified by column chromatography.<smiles>CCC(=O)OC1CCC(=O)NC1</smiles>

6-oxopiperidin-3-yl propionate (4aa)

$24{ }^{\circ} \mathrm{C}$ was applied.

Yield: $70 \%,>20: 1 \mathrm{rr}$. White solid. $\mathbf{R}_{f}=0.55(\mathrm{MeOH} / \mathrm{DCM} 1: 10)$.

${ }^{1} \mathrm{H}$ NMR $\left(500 \mathrm{MHz}, \mathrm{CDCl}_{3}\right) \delta 5.81$ (brs, $1 \mathrm{H}$ ), 5.18 (dq, J = 6.6, $3.5 \mathrm{~Hz}, 1 \mathrm{H}$ ), 3.56 (ddd, J = 12.9, 3.7, $1.1 \mathrm{~Hz}, 1 \mathrm{H}$ ), 3.42 (dtd, J = 13.0, 3.4, $1.8 \mathrm{~Hz}, 1 \mathrm{H}$ ), 2.56 (ddd, J = 17.3, 10.5, $6.5 \mathrm{~Hz}, 1 \mathrm{H}$ ), 2.43 (ddd, J $=17.9,6.4,4.4 \mathrm{~Hz}, 1 \mathrm{H}), 2.37(\mathrm{q}, \mathrm{J}=7.6 \mathrm{~Hz}, 2 \mathrm{H}), 2.15-2.05(\mathrm{~m}, 1 \mathrm{H}), 2.07-1.96(\mathrm{~m}, 1 \mathrm{H}), 1.16(\mathrm{t}, \mathrm{J}$ $=7.6 \mathrm{~Hz}, 3 \mathrm{H})$.

${ }^{13} \mathrm{C}$ NMR $\left(126 \mathrm{MHz}, \mathrm{CDCl}_{3}\right) \delta 173.8,171.0,65.2,46.2,27.7,27.2,25.1,9.0$.

IR (neat, $\mathrm{cm}^{-1}$ ) v 3212, 2930, 1727, 1656, 1495, 1371, 1179, 1082, 942, 856, 807.

LRMS (ESI+APCI) $\mathrm{m} / \mathrm{z}\left[\mathrm{C}_{8} \mathrm{H}_{14} \mathrm{NO}_{3}\right]^{+}\left([\mathrm{M}+\mathrm{H}]^{+}\right)$calculated 172.1, found 172.1 .<smiles>CC(C)CCC(=O)OC1CCC(=O)NC1</smiles>

6-oxopiperidin-3-yl 4-methylpentanoate (4ab)

Yield: $86 \%,>20: 1 \mathrm{rr}$. White solid. $\mathbf{R}_{f}=0.55$ (MeOH/DCM 1:10).

${ }^{1} \mathrm{H}$ NMR $\left(500 \mathrm{MHz}, \mathrm{CDCl}_{3}\right) \delta 6.19$ (brs, $\left.1 \mathrm{H}\right), 5.18$ (dq, J = 6.7, $3.5 \mathrm{~Hz}, 1 \mathrm{H}$ ), 3.56 (ddd, J = 13.0, 3.8, $1.4 \mathrm{~Hz}, 1 \mathrm{H}$ ), $3.42(\mathrm{dtd}, \mathrm{J}=13.0,3.3,1.6 \mathrm{~Hz}, 1 \mathrm{H}), 2.56$ (ddd, J = 17.3, 10.4, $6.6 \mathrm{~Hz}, 1 \mathrm{H}$ ), 2.44 (ddd, J $=17.9,6.4,4.4 \mathrm{~Hz}, 1 \mathrm{H}), 2.39-2.32(\mathrm{~m}, 2 \mathrm{H}), 2.14-2.06(\mathrm{~m}, 1 \mathrm{H}), 2.06-1.98(\mathrm{~m}, 1 \mathrm{H}), 1.63-1.51$ (m, 3H), $0.92(\mathrm{~d}, \mathrm{~J}=6.4 \mathrm{~Hz}, 6 \mathrm{H})$.

${ }^{13} \mathrm{C}$ NMR $(126 \mathrm{MHz}, \mathrm{CDCl} 3) \delta 173.4,171.2,65.1,46.1,33.7,32.5,27.7,27.2,25.1,22.2$.

IR $\left(\right.$ neat, $\left.\mathrm{cm}^{-1}\right)$ v 3266, 2956, 1723, 1655, 1626, 1496, 1365, 1323, 1165, 1069, 957, 792, 501. LRMS (ESI+APCI) m/z [ $\left.\mathrm{C}_{11} \mathrm{H}_{20} \mathrm{NO}_{3}\right]^{+}\left([\mathrm{M}+\mathrm{H}]^{+}\right)$calculated 214.1, found 214.2.<smiles>O=C1CCC(OC(=O)CCC2CCCCC2)CN1</smiles>

6-oxopiperidin-3-yl 3-cyclohexylpropanoate (4ac)

Yield: 88\%, >20:1 rr. White solid. $\mathbf{R}_{f}=0.65(\mathrm{MeOH} / \mathrm{DCM} 1: 10)$. 
${ }^{1} \mathrm{H}$ NMR $\left(500 \mathrm{MHz}, \mathrm{CDCl}_{3}\right) \delta 6.14$ (brs, $\left.1 \mathrm{H}\right), 5.18(\mathrm{dq}, \mathrm{J}=6.6,3.5 \mathrm{~Hz}, 1 \mathrm{H}), 3.60-3.52(\mathrm{~m}, 1 \mathrm{H}), 3.42$ (dtd, J = 13.0, 3.4, 1.6 Hz, 1H), 2.56 (ddd, J = 17.3, 10.4, 6.5 Hz, 1H), 2.44 (ddd, J = 17.9, 6.4, $4.4 \mathrm{~Hz}$, $1 \mathrm{H}), 2.39-2.32(\mathrm{~m}, 2 \mathrm{H}), 2.14-2.06(\mathrm{~m}, 1 \mathrm{H}), 2.06-1.97(\mathrm{~m}, 1 \mathrm{H}), 1.76-1.63(\mathrm{~m}, 5 \mathrm{H}), 1.58-1.50$ $(\mathrm{m}, 2 \mathrm{H}), 1.29-1.11(\mathrm{~m}, 4 \mathrm{H}), 0.97-0.85(\mathrm{~m}, 2 \mathrm{H})$.

${ }^{13} \mathrm{C} \mathrm{NMR}\left(126 \mathrm{MHz}, \mathrm{CDCl}_{3}\right) \delta 173.5,171.2,65.1,46.1,37.2,32.9,32.3,32.0,27.2,26.5,26.2,25.1$. IR (neat, $\mathrm{cm}^{-1}$ ) v 3273, 2921, 2850, 1721, 1655, 1628, 1495, 1322, 1205, 1163, 1124, 791.

LRMS (ESI+APCI) m/z $\left[\mathrm{C}_{14} \mathrm{H}_{24} \mathrm{NO}_{3}\right]^{+}\left([\mathrm{M}+\mathrm{H}]^{+}\right)$calculated 254.2, found 254.2.<smiles>CC(C)C(=O)OC1CCC(=O)NC1</smiles>

6-oxopiperidin-3-yl isobutyrate (4ad)

$2.5 \mathrm{~mol} \%$ of $\left[\mathrm{Cp}^{* \text { sdpCF3 }} \mid \mathrm{rCl}_{2}\right]_{2}$ and 1.0 equivalent of CsOPiv were applied.

Yield: $38 \%, 18: 1 \mathrm{rr}$ (65\% conv.) White solid. $\mathbf{R}_{f}=0.35$ (MeOH/EA 1:20).

${ }^{1} \mathrm{H}$ NMR $\left(500 \mathrm{MHz}, \mathrm{CDCl}_{3}\right) \delta 5.93(\mathrm{~s}, 1 \mathrm{H}), 5.18(\mathrm{dq}, J=6.6,3.5 \mathrm{~Hz}, 1 \mathrm{H}$ ), 3.57 (ddd, $J=13.0,3.9,1.4$ $\mathrm{Hz}, 1 \mathrm{H}$ ), $3.42(\mathrm{dtd}, J=13.0,3.3,1.7 \mathrm{~Hz}, 1 \mathrm{H}), 2.63-2.53(\mathrm{~m}, 2 \mathrm{H}), 2.44$ (ddd, $J=17.9,6.4,4.3 \mathrm{~Hz}$, $1 \mathrm{H}), 2.15-2.08(\mathrm{~m}, 1 \mathrm{H}), 2.07-1.99(\mathrm{~m}, 1 \mathrm{H}), 1.21(\mathrm{~d}, J=3.1 \mathrm{~Hz}, 3 \mathrm{H}), 1.19(\mathrm{~d}, J=3.0 \mathrm{~Hz}, 3 \mathrm{H})$.

${ }^{13} \mathrm{C} \mathrm{NMR}\left(126 \mathrm{MHz}, \mathrm{CDCl}_{3}\right) \delta 176.4,171.1,65.0,46.1,34.1,27.1,25.1,18.9,18.9$.

IR (neat, $\left.\mathrm{cm}^{-1}\right)$ v 3262, 2970, 2935, 1726, 1655, 1630, 1496, 1363, 1323, 1192, 1154.

LRMS (ESI+APCI) m/z $\left[\mathrm{C}_{9} \mathrm{H}_{16} \mathrm{NO}_{3}\right]^{+}\left([\mathrm{M}+\mathrm{H}]^{+}\right)$calculated 186.1, found 186.2.<smiles>CC(=O)CCC(=O)OC1CCC(=O)NC1</smiles>

methyl (6-oxopiperidin-3-yl) succinate (4ae)

Yield: 84\%, >20:1. White solid. $\mathbf{R}_{f}=0.50(\mathrm{MeOH} / \mathrm{DCM} 1: 10)$.

${ }^{1} \mathrm{H}$ NMR $\left(500 \mathrm{MHz}, \mathrm{CDCl}_{3}\right) \delta 6.08$ (brs, $\left.1 \mathrm{H}\right), 5.21(\mathrm{dq}, \mathrm{J}=6.7,3.5 \mathrm{~Hz}, 1 \mathrm{H}), 3.72(\mathrm{~s}, 3 \mathrm{H}), 3.56$ (ddd, J $=13.1,3.9,1.5 \mathrm{~Hz}, 1 \mathrm{H}), 3.44(\mathrm{dtd}, \mathrm{J}=13.1,3.4,1.7 \mathrm{~Hz}, 1 \mathrm{H}), 2.73-2.63(\mathrm{~m}, 4 \mathrm{H}), 2.57$ (ddd, J = 17.3, $10.5,6.5 \mathrm{~Hz}, 1 \mathrm{H}$ ), 2.43 (ddd, J = 17.9, 6.5, 4.4 Hz, 1H), $2.17-2.08(\mathrm{~m}, 1 \mathrm{H}), 2.07-1.98(\mathrm{~m}, 1 \mathrm{H})$.

${ }^{13} \mathrm{C} \mathrm{NMR}\left(126 \mathrm{MHz}, \mathrm{CDCl}_{3}\right) \delta 172.6,171.6,171.0,65.8,51.9,46.0,29.2,28.8,27.1,25.0$.

IR $\left(\right.$ neat, $\mathrm{cm}^{-1}$ ) v 2949, 1729, 1662, 1494, 1438, 1364, 1320, 1208, 1159, 991, 849.

LRMS (ESI+APCI) m/z $\left[\mathrm{C}_{10} \mathrm{H}_{16} \mathrm{NO}_{5}\right]^{+}\left([\mathrm{M}+\mathrm{H}]^{+}\right)$calculated 230.1, found 230.1 .<smiles>O=C1CCC(OC(=O)c2ccccc2)CN1</smiles> 
6-oxopiperidin-3-yl benzoate (4af)

1.0 equivalent of CsOPiv was used.

Yield: $55 \%, 12: 1 \mathrm{rr}$. White solid. $\mathbf{R}_{f}=0.60(\mathrm{MeOH} / \mathrm{DCM} 1: 10)$.

${ }^{1} \mathrm{H}$ NMR $\left(500 \mathrm{MHz}, \mathrm{CDCl}_{3}\right) \delta 8.08-8.04(\mathrm{~m}, 2 \mathrm{H}), 7.65-7.57(\mathrm{~m}, 1 \mathrm{H}), 7.51-7.45(\mathrm{~m}, 2 \mathrm{H}), 6.10$ (brs, $1 \mathrm{H}$ ), 5.46 (dq, J = 6.4, $3.4 \mathrm{~Hz}, 1 \mathrm{H}$ ), 3.70 (ddd, J = 13.1, 3.8, $1.3 \mathrm{~Hz}, 1 \mathrm{H}$ ), 3.60 (dtd, J = 13.2, 3.3, $1.7 \mathrm{~Hz}, 1 \mathrm{H}$ ), 2.70 (ddd, J = 17.6, 10.8, $6.5 \mathrm{~Hz}, 1 \mathrm{H}$ ), 2.53 (ddd, J = 17.9, 6.4, $4.1 \mathrm{~Hz}, 1 \mathrm{H}$ ), $2.31-2.24$ (m, 1H), $2.19-2.11(\mathrm{~m}, 1 \mathrm{H})$.

${ }^{13} \mathrm{C}$ NMR $\left(126 \mathrm{MHz}, \mathrm{CDCl}_{3}\right) \delta 171.1,165.8,133.4,129.7,128.5,65.8,46.3,27.2,25.2$.

IR $\left(\right.$ neat, $\mathrm{cm}^{-1}$ ) v 3254, 2960, 1714, 1661, 1495, 1360, 1274, 1112, 1061, 803, 709.

LRMS $\left(\right.$ ESI+APCl) $\mathrm{m} / \mathrm{z}\left[\mathrm{C}_{12} \mathrm{H}_{14} \mathrm{NO}_{3}\right]^{+}\left([\mathrm{M}+\mathrm{H}]^{+}\right)$calculated 220.1, found 220.1 .<smiles>O=C1CCC(OC(=O)CCCl)CN1</smiles>

6-oxopiperidin-3-yl 3-chloropropanoate (4ak)

Yield: $79 \%,>20: 1 \mathrm{rr}$. White solid. $\mathbf{R}_{f}=0.60(\mathrm{MeOH} / \mathrm{DCM} 1: 10)$.

${ }^{1} \mathrm{H}$ NMR (500 MHz, CDCl $)_{3} \delta 6.30(\mathrm{brs}, 1 \mathrm{H}), 5.26(\mathrm{dq}, \mathrm{J}=6.6,3.5 \mathrm{~Hz}, 1 \mathrm{H}), 3.79(\mathrm{t}, \mathrm{J}=6.5 \mathrm{~Hz}, 2 \mathrm{H})$, 3.59 (ddd, J = 13.2, 3.8, 1.4 Hz, 1H), $3.46(\mathrm{dtd}, \mathrm{J}=13.1,3.4,1.7 \mathrm{~Hz}, 1 \mathrm{H}), 2.85(\mathrm{t}, \mathrm{J}=6.4 \mathrm{~Hz}, 2 \mathrm{H}$ ), 2.58 (ddd, J = 17.4, 10.5, 6.5 Hz, 1H), 2.45 (ddd, J = 17.9, 6.5, 4.3 Hz, 1H), $2.18-2.11(\mathrm{~m}, 1 \mathrm{H}$ ), 2.09 $-2.00(\mathrm{~m}, 1 \mathrm{H})$.

${ }^{13} \mathrm{C} \mathrm{NMR}\left(126 \mathrm{MHz}, \mathrm{CDCl}_{3}\right) \delta 171.1,169.7,66.1,46.0,39.0,37.6,27.1,25.0$.

IR (neat, $\mathrm{cm}^{-1}$ ) v 3261, 1732, 1655, 1495, 1366, 1324, 1206, 1156, 1071, 936, 793, 484.

LRMS (ESI+APCI) $\mathrm{m} / \mathrm{z}\left[\mathrm{C}_{8} \mathrm{H}_{13} \mathrm{ClNO}_{3}\right]^{+}\left([\mathrm{M}+\mathrm{H}]^{+}\right)$calculated 206.1, found 206.1, 208.1.<smiles>O=C1CCC(OC(=O)CCBr)CN1</smiles>

6-oxopiperidin-3-yl 3-bromopropanoate (4al)

Yield: 77\%, >20:1 rr. White solid. $\mathbf{R}_{f}=0.50(\mathrm{MeOH} / \mathrm{DCM} 1: 10)$.

${ }^{1} \mathrm{H}$ NMR $\left(500 \mathrm{MHz}, \mathrm{CDCl}_{3}\right) \delta 6.13(\mathrm{brs}, 1 \mathrm{H}), 5.27(\mathrm{dq}, \mathrm{J}=6.5,3.4 \mathrm{~Hz}, 1 \mathrm{H}), 3.63-3.57(\mathrm{~m}, 3 \mathrm{H}), 3.47$ (dtd, J = 13.2, 3.3, 1.8 Hz, 1H), $2.98(\mathrm{t}, \mathrm{J}=6.6 \mathrm{~Hz}, 2 \mathrm{H}$ ), 2.59 (ddd, J = 17.5, 10.6, 6.6 Hz, 1H), 2.46 (ddd, J = 17.9, 6.5, 4.2 Hz, 1H), $2.19-2.11(\mathrm{~m}, 1 \mathrm{H}), 2.09-2.01(\mathrm{~m}, 1 \mathrm{H})$.

${ }^{13} \mathrm{C} \mathrm{NMR}\left(126 \mathrm{MHz}, \mathrm{CDCl}_{3}\right) \delta 171.0,169.9,66.1,46.0,37.7,27.1,25.8,25.0$.

IR $\left(\right.$ neat, $\left.\mathrm{cm}^{-1}\right)$ v 3220, 2923, 1731, 1662, 1495, 1176, 1129, 1066, 855.

LRMS (ESI+APCI) $\mathrm{m} / \mathrm{z}\left[\mathrm{C}_{8} \mathrm{H}_{13} \mathrm{BrNO}_{3}\right]^{+}\left([\mathrm{M}+\mathrm{H}]^{+}\right)$calculated 250.0, found 250.0, 252.0. 
<smiles>O=C1CCC(OC(=O)CCc2ccc(O)cc2)CN1</smiles>

6-oxopiperidin-3-yl 3-(4-hydroxyphenyl)propanoate (4am)

Yield: $77 \%, 18: 1 \mathrm{rr}$. White solid. $\mathbf{R}_{f}=0.45(\mathrm{MeOH} / \mathrm{DCM} 1: 10)$.

${ }^{1} \mathrm{H}$ NMR $\left(500 \mathrm{MHz}, \mathrm{CDCl}_{3}\right) \delta 7.44$ (brs, $\left.1 \mathrm{H}\right), 7.06-7.02(\mathrm{~m}, 2 \mathrm{H}), 6.82-6.78(\mathrm{~m}, 2 \mathrm{H}), 6.53$ (brs, $\left.1 \mathrm{H}\right)$, $5.12(\mathrm{dq}, \mathrm{J}=6.0,3.2 \mathrm{~Hz}, 1 \mathrm{H}), 3.42(\mathrm{dd}, \mathrm{J}=13.6,3.5 \mathrm{~Hz}, 1 \mathrm{H}), 3.14(\mathrm{dtd}, \mathrm{J}=13.4,3.2,1.8 \mathrm{~Hz}, 1 \mathrm{H}$ ), $2.95-2.84(\mathrm{~m}, 2 \mathrm{H}), 2.64(\mathrm{t}, \mathrm{J}=7.3 \mathrm{~Hz}, 2 \mathrm{H}), 2.43-2.31(\mathrm{~m}, 2 \mathrm{H}), 2.03-1.96(\mathrm{~m}, 1 \mathrm{H}), 1.95-1.87$ $(\mathrm{m}, 1 \mathrm{H})$.

${ }^{13} \mathrm{C}$ NMR $\left(126 \mathrm{MHz}, \mathrm{CDCl}_{3}\right) \delta 172.4,172.0,154.9,131.3,129.4,115.7,64.8,46.0,36.2,30.4,26.6$, 24.6.

IR (neat, $\mathrm{cm}^{-1}$ ) v 3221, 2926, 1730, 1654, 1515, 1365, 1245, 1157, 830.

LRMS (ESI+APCI) $\mathrm{m} / \mathrm{z}\left[\mathrm{C}_{14} \mathrm{H}_{18} \mathrm{NO}_{4}\right]^{+}\left([\mathrm{M}+\mathrm{H}]^{+}\right)$calculated 264.1, found 264.1.<smiles>O=C1CCC(OC(=O)COc2ccccc2)CN1</smiles>

6-oxopiperidin-3-yl 2-phenoxyacetate (4an)

Yield: 80\%, 18:1 rr. White solid. $\mathbf{R}_{f}=0.70(\mathrm{MeOH} / \mathrm{DCM} 1: 10)$.

${ }^{1} \mathrm{H}$ NMR $\left(500 \mathrm{MHz}, \mathrm{CDCl}_{3}\right) \delta 7.32(\mathrm{dd}, \mathrm{J}=7.5 \mathrm{~Hz}, 2 \mathrm{H}), 7.03(\mathrm{t}, \mathrm{J}=7.4 \mathrm{~Hz}, 1 \mathrm{H}), 6.92(\mathrm{~d}, \mathrm{~J}=8.0 \mathrm{~Hz}$, 2H), 6.32 (brs, $1 \mathrm{H}), 5.34-5.29(\mathrm{~m}, 1 \mathrm{H}), 4.69(\mathrm{~s}, 2 \mathrm{H}), 3.58(\mathrm{dd}, \mathrm{J}=13.5,3.7 \mathrm{~Hz}, 1 \mathrm{H}), 3.49-3.41(\mathrm{~m}$, $1 \mathrm{H}), 2.51-2.37(\mathrm{~m}, 2 \mathrm{H}), 2.16-2.00(\mathrm{~m}, 2 \mathrm{H})$.

${ }^{13} \mathrm{C} \mathrm{NMR}\left(126 \mathrm{MHz}, \mathrm{CDCl}_{3}\right) \delta 170.9,168.5,157.6,129.7,122.0,114.6,66.5,65.3,45.9,26.9,25.0$. IR (neat, $\mathrm{cm}^{-1}$ ) v 2930, 1756, 1661, 1601, 1494, 1189, 1090, 961, 836, 755.

LRMS (ESI+APCI) $\mathrm{m} / \mathrm{z}\left[\mathrm{C}_{13} \mathrm{H}_{16} \mathrm{NO}_{4}\right]^{+}\left([\mathrm{M}+\mathrm{H}]^{+}\right)$calculated 250.1, found 250.1 .<smiles>O=C([O-])NCC(=O)OC1CCC(=O)NC1</smiles>

6-oxopiperidin-3-yl (tert-butoxycarbonyl)glycinate (4ao)

1.0 equivalent of $\mathrm{CsOPiv}$ was used.

Yield: $68 \%,>20: 1 \mathrm{rr}$. White solid. $\mathbf{R}_{f}=0.45(\mathrm{MeOH} / \mathrm{DCM} 1: 10)$.

${ }^{1} \mathrm{H}$ NMR $\left(500 \mathrm{MHz}, \mathrm{CDCl}_{3}\right) \delta 6.26$ (brs, $\left.1 \mathrm{H}\right), 5.25$ (dq, J = 6.7, 3.6 Hz, 1H), 5.06 (brs, 1H), $3.99-3.84$ $(\mathrm{m}, 2 \mathrm{H}), 3.58(\mathrm{dd}, \mathrm{J}=12.1,3.6 \mathrm{~Hz}, 1 \mathrm{H}), 3.49-3.41(\mathrm{~m}, 1 \mathrm{H}), 2.56$ (ddd, J = 17.2, 10.3, $6.5 \mathrm{~Hz}, 1 \mathrm{H}$ ), 2.43 (ddd, J = 17.9, 6.5, $4.5 \mathrm{~Hz}, 1 \mathrm{H}), 2.17-2.09(\mathrm{~m}, 1 \mathrm{H}), 2.09-2.01(\mathrm{~m}, 1 \mathrm{H})$.

${ }^{13} \mathrm{C} \mathrm{NMR}\left(126 \mathrm{MHz}, \mathrm{CDCl}_{3}\right) \delta 171.0,169.8,155.7,80.2,66.5,45.9,42.5,28.3,27.0,25.1$.

IR $\left(\right.$ neat, $\left.\mathrm{cm}^{-1}\right)$ v 3304, 2973, 1701, 1663, 1500, 1366, 1252, 1163, 1072, 961, 861.

LRMS (ESI+APCI) m/z $\left[\mathrm{C}_{12} \mathrm{H}_{20} \mathrm{~N}_{2} \mathrm{O}_{5} \mathrm{Na}\right]^{+}\left([\mathrm{M}+\mathrm{Na}]^{+}\right)$calculated 295.1, found 295.1. 


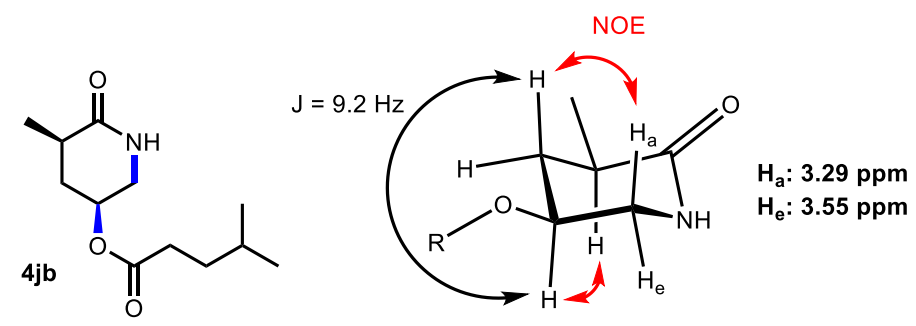

5-methyl-6-oxopiperidin-3-yl 4-methylpentanoate (4jb)

$40{ }^{\circ} \mathrm{C}$ was applied.

Yield: $50 \%,>20: 1 \mathrm{rr},>20: 1 \mathrm{dr}$. White solid. $\mathbf{R}_{f}=0.70(\mathrm{MeOH} / \mathrm{DCM} 1: 10)$.

${ }^{1} \mathrm{H}$ NMR $\left(500 \mathrm{MHz}, \mathrm{CDCl}_{3}\right) \delta 6.23$ (brs, $1 \mathrm{H}$ ), 5.12 (ddt, J = 9.4, 7.2, $4.8 \mathrm{~Hz}, 1 \mathrm{H}$ ), 3.55 (dddd, J = 12.0, 4.8, 3.2, 1.4 Hz, 1H), 3.29 (ddd, J = 12.1, 7.2, 2.2 Hz, 1H), 2.48 (dp, J = 10.6, 7.0 Hz, $1 \mathrm{H}$ ), $2.35-$ $2.26(\mathrm{~m}, 3 \mathrm{H}), 1.68(\mathrm{ddd}, \mathrm{J}=13.3,10.7,9.2 \mathrm{~Hz}, 1 \mathrm{H}), 1.62-1.49(\mathrm{~m}, 3 \mathrm{H}), 1.28(\mathrm{~d}, \mathrm{~J}=7.2 \mathrm{~Hz}, 3 \mathrm{H})$, $0.91(\mathrm{~d}, \mathrm{~J}=6.3 \mathrm{~Hz}, 6 \mathrm{H})$.

${ }^{13} \mathrm{C}$ NMR $\left(126 \mathrm{MHz}, \mathrm{CDCl}_{3}\right) \delta 174.7,173.3,66.6,45.7,34.5,33.7,33.6,32.4,27.7,22.2,17.1$.

IR (neat, $\mathrm{cm}^{-1}$ ) v 2955, 2870, 1733, 1665, 1465, 1385, 1261, 1172, 1102, 773.

LRMS (ESI+APCI) $\mathrm{m} / \mathrm{z}\left[\mathrm{C}_{12} \mathrm{H}_{22} \mathrm{NO}_{3}\right]^{+}\left([\mathrm{M}+\mathrm{H}]^{+}\right)$calculated 228.2, found 228.2.<smiles>CC(C)CCC(=O)OC1CC(C)C(=O)N[C@@H]1O</smiles>

Prepared from $1 \mathrm{j}-\mathrm{d}_{\mathbf{1}}$ with $5 \mathrm{~mol} \%$ of $\left[\mathrm{Cp}{ }^{* \mathrm{bispCF}} \mathrm{IrCl}_{2}\right]_{2}$.

${ }^{1} \mathrm{H}$ NMR $\left(500 \mathrm{MHz}, \mathrm{CDCl}_{3}\right) \delta 5.66(\mathrm{~s}, 1 \mathrm{H}), 5.13$ (ddd, $\left.J=9.2,7.2,4.6 \mathrm{~Hz}, 1 \mathrm{H}\right), \mathbf{3 . 2 8}(\mathrm{d}, \boldsymbol{J}=\mathbf{7 . 2 ~ H z}, \mathbf{1 H})$, $2.53-2.46(\mathrm{~m}, 1 \mathrm{H}), 2.37-2.28(\mathrm{~m}, 3 \mathrm{H}), 1.70(\mathrm{ddd}, J=13.3,10.8,9.2 \mathrm{~Hz}, 1 \mathrm{H}), 1.59-1.51(\mathrm{~m}, 3 \mathrm{H})$, $1.29(\mathrm{~d}, J=7.1 \mathrm{~Hz}, 3 \mathrm{H}), 0.93(\mathrm{~s}, 3 \mathrm{H}), 0.92(\mathrm{~s}, 3 \mathrm{H})$.<smiles>CC(C)CCC(=O)OC1CNC(=O)C(Cc2ccc(Br)cc2)C1</smiles>

5-(4-(benzyloxy)benzyl)-6-oxopiperidin-3-yl 4-methylpentanoate (4kb) $40{ }^{\circ} \mathrm{C}$ was applied.

Yield: $52 \%,>20: 1 \mathrm{rr},>20: 1 \mathrm{dr}$. Colorless oil. $\mathbf{R}_{f}=0.80(\mathrm{MeOH} / \mathrm{DCM} 1: 10)$.

${ }^{1}$ H NMR $\left(500 \mathrm{MHz}, \mathrm{CDCl}_{3}\right) \delta 7.48-7.44(\mathrm{~m}, 2 \mathrm{H}), 7.44-7.38(\mathrm{~m}, 2 \mathrm{H}), 7.38-7.32(\mathrm{~m}, 1 \mathrm{H}), 7.16-$ $7.11(\mathrm{~m}, 2 \mathrm{H}), 6.96-6.92(\mathrm{~m}, 2 \mathrm{H}), 6.03$ (brs, $1 \mathrm{H}), 5.11-5.02(\mathrm{~m}, 3 \mathrm{H}), 3.53$ (dddd, J = 11.7, 5.0, 3.4, $1.5 \mathrm{~Hz}, 1 \mathrm{H}$ ), 3.33 (dd, J = 13.8, 4.1 Hz, 1H), 3.22 (ddd, J = 11.9, 7.6, $1.8 \mathrm{~Hz}, 1 \mathrm{H}$ ), 2.75 (dd, J = 13.8, $9.6 \mathrm{~Hz}, 1 \mathrm{H}$ ), 2.62 (tdd, J = 10.2, 6.3, $4.1 \mathrm{~Hz}, 1 \mathrm{H}$ ), $2.33-2.28$ (m, 2H), 2.06 (dddd, J = 13.2, 6.2, 4.4, $1.5 \mathrm{~Hz}, 1 \mathrm{H}), 1.69-1.63(\mathrm{~m}, 1 \mathrm{H}), 1.60-1.49(\mathrm{~m}, 3 \mathrm{H}), 0.91(\mathrm{~d}, \mathrm{~J}=6.4 \mathrm{~Hz}, 6 \mathrm{H})$.

${ }^{13} \mathrm{C} \mathrm{NMR}\left(126 \mathrm{MHz}, \mathrm{CDCl}_{3}\right) \delta 173.2,173.2,157.5,137.1,131.3,130.3,128.6,127.9,127.5,114.9$, 70.0, 66.7, 45.5, 40.5, 36.3, 33.6, 32.4, 31.0, 27.7, 22.2.

IR $\left(\right.$ neat, $\left.\mathrm{cm}^{-1}\right)$ v 2954, 1733, 1664, 1509, 1237, 1173, 1104, 1020, 809, 736, 696.

LRMS (ESI+APCI) $\mathrm{m} / \mathrm{z}\left[\mathrm{C}_{25} \mathrm{H}_{32} \mathrm{NO}_{4}\right]^{+}\left([\mathrm{M}+\mathrm{H}]^{+}\right)$calculated 410.2, found 410.3 . 


\section{Synthesis of $\gamma$ and $\delta$-lactones}

\section{General Procedure:}

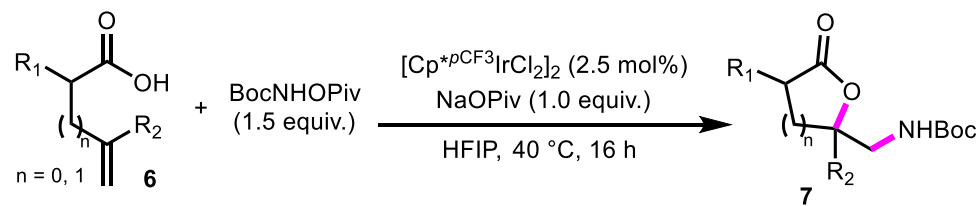

Alkenyl carboxylates (0.10 mmol), BocNHOPiv (0.15 mmol, 1.5 equiv.), NaOPiv (0.10 mmol, 1.0 equiv.) and HFIP (170 $\mu \mathrm{L})$ were combined in a 1 dram vial with a magnetic stir bar. In a separate vial, $\left[\mathrm{Cp}^{*{ }^{p C F 3}} \mathrm{IrCl}_{2}\right]_{2}$ or $\left[\mathrm{Cp}^{*{ }^{\mathrm{sd} p C \mathrm{CF} 3}} \mathrm{IrCl}_{2}\right]_{2}(0.0025 \mathrm{mmol}, 2.5 \mathrm{~mol} \%)$ was dissolved in HFIP $(170 \mu \mathrm{L})$, and then transferred to the first vial. The reaction was stirred at $40{ }^{\circ} \mathrm{C}$. After 16 hours, the reaction mixture was filtered through a pad of Celite ${ }^{\circledR}$, and the filtrate was concentrated by rotary evaporation. A crude ${ }^{1} \mathrm{H}$ NMR spectrum was collected with mesitylene as internal standard. The crude mixture was then purified by column chromatography.

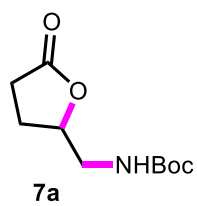

tert-butyl ((5-oxotetrahydrofuran-2-yl)methyl)carbamate (7a)

Yield: 95\%. White solid. $\mathbf{R}_{f}=0.35$ (EA/HEX 2:3).

${ }^{1} \mathrm{H}$ NMR (500 MHz, $\mathrm{CDCl}_{3}$ ) $\delta 4.96(\mathrm{~s}, 1 \mathrm{H}), 4.61$ (qd, J = 7.1, 3.3 Hz, 1H), 3.53 (ddd, J = 14.8, 6.9, 3.3 $\mathrm{Hz}, 1 \mathrm{H}), 3.28(\mathrm{dt}, \mathrm{J}=14.7,6.2 \mathrm{~Hz}, 1 \mathrm{H}), 2.56(\mathrm{dd}, \mathrm{J}=9.5,7.2 \mathrm{~Hz}, 2 \mathrm{H}), 2.30(\mathrm{dq}, \mathrm{J}=13.2,7.1 \mathrm{~Hz}, 1 \mathrm{H})$, 1.98 (dtd, J = 13.0, 9.5, 7.8 Hz, 1H), $1.45(\mathrm{~s}, 9 \mathrm{H})$.

${ }^{13} \mathrm{C}$ NMR $\left(126 \mathrm{MHz}, \mathrm{CDCl}_{3}\right) \delta 176.8,156.0,79.9,79.7,44.0,28.6,28.3,24.5$.

IR (neat, cm-1) v 3351, 2976, 2931, 1771, 1696, 1518, 1365, 1249, 1156, 1063, 918, 649.

HRMS (ESI) m/z [C10H17NO4Na $]^{+}\left([\mathrm{M}+\mathrm{Na}]^{+}\right)$calculated 238.1055, found 238.1083.

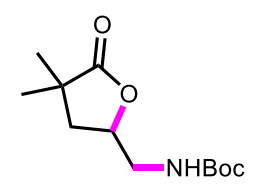

$7 \mathbf{b}$

tert-butyl ((4,4-dimethyl-5-oxotetrahydrofuran-2-yl)methyl)carbamate (7b)

Yield: $90 \%$. Light yellow solid. $\mathbf{R}_{f}=0.40$ (EA/HEX 2:3).

${ }^{1} \mathrm{H}$ NMR (400 MHz, CDCl $\left.{ }_{3}\right) \delta 4.95(\mathrm{~s}, 1 \mathrm{H}), 4.60-4.48(\mathrm{~m}, 1 \mathrm{H}), 3.58$ (ddd, $\left.J=14.6,6.9,3.0 \mathrm{~Hz}, 1 \mathrm{H}\right)$, $3.20(\mathrm{dt}, J=14.6,6.2 \mathrm{~Hz}, 1 \mathrm{H}), 2.12(\mathrm{dd}, J=12.9,6.2 \mathrm{~Hz}, 1 \mathrm{H}), 1.81(\mathrm{dd}, J=12.9,10.0 \mathrm{~Hz}, 1 \mathrm{H}), 1.45$ (s, 9H), $1.29(\mathrm{~s}, 3 \mathrm{H}), 1.28(\mathrm{~s}, 3 \mathrm{H})$.

${ }^{13} \mathrm{C}$ NMR $\left(101 \mathrm{MHz}^{\mathrm{CDCl}}\right)_{3} \delta 181.6,156.0,79.9,76.1,44.1,40.3,39.7,28.3,24.9,24.6$

IR (neat, $\mathrm{cm}^{-1}$ ) v 3356, 2972, 2932, 2873, 1767, 1698, 1516, 1366, 1248, 1161, 1130, 1053, 916.

HRMS (ESI) $\mathrm{m} / \mathrm{z}\left[\mathrm{C}_{12} \mathrm{H}_{21} \mathrm{NO}_{4} \mathrm{Na}\right]^{+}\left([\mathrm{M}+\mathrm{Na}]^{+}\right)$calculated 266.1368, found 266.1394.

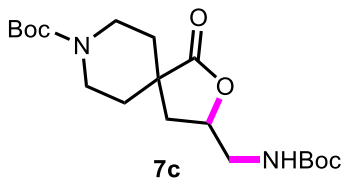


tert-butyl 3-(((tert-butoxycarbonyl)amino)methyl)-1-oxo-2-oxa-8-azaspiro[4.5]decane-8-carboxylate (7c)

Yield: $95 \%$. Light yellow oil. $\mathbf{R}_{f}=0.20$ (EA/HEX 2:3).

${ }^{1} \mathrm{H}$ NMR $\left(400 \mathrm{MHz}, \mathrm{CDCl}_{3}\right) \delta 4.97(\mathrm{~s}, 1 \mathrm{H}), 4.66-4.50(\mathrm{~m}, 1 \mathrm{H}), 4.08-3.90(\mathrm{~m}, 1 \mathrm{H}), 3.90-3.76(\mathrm{~m}$, $1 \mathrm{H}), 3.63-3.49(\mathrm{~m}, 1 \mathrm{H}), 3.25(\mathrm{dt}, J=13.9,6.2 \mathrm{~Hz}, 1 \mathrm{H}), 3.20-3.10(\mathrm{~m}, 1 \mathrm{H}), 3.10-2.97(\mathrm{~m}, 1 \mathrm{H})$, 2.30 (dd, $J=13.2,6.5 \mathrm{~Hz}, 1 \mathrm{H}$ ), 1.94 (ddd, $J=13.9,10.0,4.2 \mathrm{~Hz}, 1 \mathrm{H}), 1.88-1.71(\mathrm{~m}, 2 \mathrm{H}), 1.64-$ $1.49(\mathrm{~m}, 2 \mathrm{H}), 1.46(\mathrm{~s}, 9 \mathrm{H}), 1.45(\mathrm{~s}, 9 \mathrm{H})$.

${ }^{13} \mathrm{C}$ NMR $\left(101 \mathrm{MHz}, \mathrm{CDCl}_{3}\right) \delta 179.6,156.0,154.5,80.0,79.9,76.4,44.1,42.9,40.3,39.6,35.8$, $33.3,31.8,28.4,28.3$.

IR (neat, $\mathrm{cm}^{-1}$ ) v 3346, 2975, 2931, 2867, 1765, 1690, 1424, 1365, 1248, 1152, $1049,863$.

HRMS (ESI) $\mathrm{m} / \mathrm{z}\left[\mathrm{C}_{19} \mathrm{H}_{32} \mathrm{~N}_{2} \mathrm{O}_{6} \mathrm{Na}\right]^{+}\left([\mathrm{M}+\mathrm{Na}]^{+}\right)$calculated 407.2158, found 407.2176.

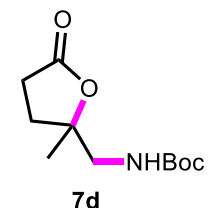

tert-butyl ((2-methyl-5-oxotetrahydrofuran-2-yl)methyl)carbamate (7d)

Yield: $96 \%$. White solid. $\mathbf{R}_{f}=0.35$ (EA/HEX 2:3).

${ }^{1} \mathrm{H}$ NMR $\left(500 \mathrm{MHz}, \mathrm{CDCl}_{3}\right) \delta 4.89(\mathrm{~s}, 1 \mathrm{H}), 3.39$ (dd, $J=14.6,7.0 \mathrm{~Hz}, 1 \mathrm{H}$ ), 3.32 (dd, $J=14.5,6.2 \mathrm{~Hz}$, 1H), $2.70-2.55(\mathrm{~m}, 2 \mathrm{H}), 2.25(\mathrm{dt}, J=13.0,8.8 \mathrm{~Hz}, 1 \mathrm{H}), 1.97$ (ddd, $J=13.1,9.7,6.3 \mathrm{~Hz}, 1 \mathrm{H}), 1.46(\mathrm{~s}$, 9H), $1.40(\mathrm{~s}, 3 \mathrm{H})$.

${ }^{13} \mathrm{C}$ NMR $\left(126 \mathrm{MHz}, \mathrm{CDCl}_{3}\right) \delta 176.5,156.2,86.2,79.9,48.3,30.1,29.2,28.3,24.0$.

IR (neat, $\mathrm{cm}^{-1}$ ) v 3350, 2977, 2932, 1768, 1707, 1520, 1366, 1247, 1164, 947, 932, 642.

HRMS (ESI) $\mathrm{m} / \mathrm{z}\left[\mathrm{C}_{11} \mathrm{H}_{19} \mathrm{NO}_{4} \mathrm{Na}\right]^{+}\left([\mathrm{M}+\mathrm{Na}]^{+}\right)$calculated 252.1206, found 252.1239.

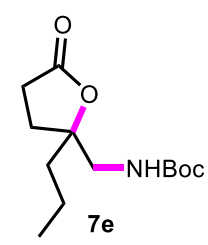

tert-butyl ((5-oxo-2-propyltetrahydrofuran-2-yl)methyl)carbamate (7e)

$5 \mathrm{~mol} \%$ of $\left[\mathrm{Cp}^{*{ }^{p C F} 3} \mid \mathrm{rCl}_{2}\right]_{2}$ was applied.

Yield: $71 \%$. Light yellow solid. $\mathbf{R}_{f}=0.40(\mathrm{EA} / \mathrm{HEX} 2: 3)$.

${ }^{1} \mathrm{H}$ NMR $\left(400 \mathrm{MHz}, \mathrm{CDCl}_{3}\right) \delta 4.84(\mathrm{~s}, 1 \mathrm{H}), 3.42$ (dd, $\left.J=14.5,7.2 \mathrm{~Hz}, 1 \mathrm{H}\right), 3.31$ (dd, $J=14.4,6.0 \mathrm{~Hz}$, $1 \mathrm{H}), 2.60(\mathrm{t}, J=8.6 \mathrm{~Hz}, 2 \mathrm{H}), 2.18(\mathrm{dt}, J=13.3,8.7 \mathrm{~Hz}, 1 \mathrm{H}), 2.03(\mathrm{dt}, J=13.3,8.5 \mathrm{~Hz}, 1 \mathrm{H}), 1.70-$ $1.61(\mathrm{~m}, 2 \mathrm{H}), 1.45(\mathrm{~s}, 9 \mathrm{H}), 1.44-1.33(\mathrm{~m}, 2 \mathrm{H}), 0.96(\mathrm{t}, J=7.3 \mathrm{~Hz}, 3 \mathrm{H})$.

${ }^{13} \mathrm{C} \mathrm{NMR}\left(101 \mathrm{MHz} \mathrm{CDCl}_{3}\right) \delta 176.8,156.3,88.5,79.9,47.0,39.6,29.3,28.3,27.8,16.6,14.4$.

IR $\left(\right.$ neat, $\mathrm{cm}^{-1}$ ) v 3351, 2964, 2934, 2874, 1770, 1709, 1523, 1366, 1248, 1166, 933.

HRMS (ESI) $\mathrm{m} / \mathrm{z}\left[\mathrm{C}_{13} \mathrm{H}_{23} \mathrm{NO}_{4} \mathrm{Na}\right]^{+}\left([\mathrm{M}+\mathrm{Na}]^{+}\right)$calculated 280.1525, found 280.1550 . 


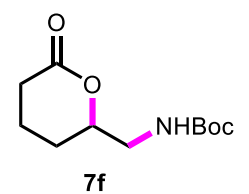

tert-butyl ((6-oxotetrahydro-2H-pyran-2-yl)methyl)carbamate (7f)

5 mol\% of $\left[\mathrm{Cp}^{*{ }^{\mathrm{sdpCF}}} \mid \mathrm{ICl}_{2}\right]_{2}$ was applied.

Yield: $85 \%$. Light yellow oil. $\boldsymbol{R}_{f}=0.20$ (EA/HEX 2:3).

${ }^{1} \mathrm{H}$ NMR $\left(500 \mathrm{MHz}, \mathrm{CDCl}_{3}\right) \delta 5.06(\mathrm{~s}, 1 \mathrm{H}), 4.39$ (ddt, $J=10.8,6.8,3.1 \mathrm{~Hz}, 1 \mathrm{H}$ ), 3.52 (ddd, $J=14.6$, 7.5, 3.1 Hz, 1H), $3.26-3.15(\mathrm{~m}, 1 \mathrm{H}), 2.66-2.57(\mathrm{~m}, 1 \mathrm{H}), 2.46$ (ddd, $J=17.8,9.1,6.9 \mathrm{~Hz}, 1 \mathrm{H}), 1.99$ $-1.83(\mathrm{~m}, 3 \mathrm{H}), 1.61-1.53(\mathrm{~m}, 1 \mathrm{H}), 1.45(\mathrm{~s}, 9 \mathrm{H})$.

${ }^{13} \mathrm{C}$ NMR $\left(126 \mathrm{MHz}, \mathrm{CDCl}_{3}\right) \delta 171.3,156.0,79.9,79.7,44.7,29.4,28.3,24.9,18.3$.

IR (neat, $\mathrm{cm}^{-1}$ ) v 3351, 2973, 2932, 1704, 1516, 1365, 1236, 1163, 1053, 935, 636

HRMS (ESI) $\mathrm{m} / \mathrm{z}\left[\mathrm{C}_{11} \mathrm{H}_{19} \mathrm{NO}_{4} \mathrm{Na}\right]^{+}\left([\mathrm{M}+\mathrm{Na}]^{+}\right)$calculated 252.1212, found 252.1239.

\section{Mechanistic study}

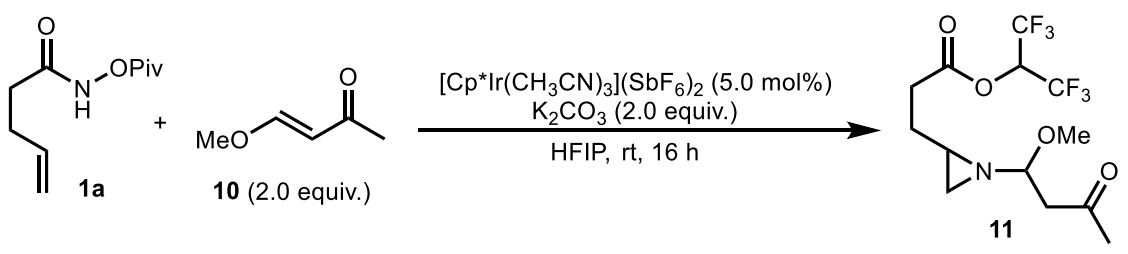

Alkenyl N- pivaloylhydroxamate 1a (12 mg, $0.06 \mathrm{mmol}$ ), trans-4-Methoxy-3-buten-2-one 10 (12 $\mu \mathrm{L}, 0.12 \mathrm{mmol}), \mathrm{K}_{2} \mathrm{CO}_{3}(13 \mathrm{mg}, 0.12 \mathrm{mmol})$ and $\mathrm{HFIP}(100 \mu \mathrm{L})$ were combined in a 1 dram vial with a magnetic stir bar. In a separate vial, $\left[\mathrm{Cp}^{*} \mathrm{Ir}\left(\mathrm{CH}_{3} \mathrm{CN}\right)_{3}\right]\left(\mathrm{SbF}_{6}\right)_{2}(2.8 \mathrm{mg}, 0.003 \mathrm{mmol})$ was dissolved in HFIP $(100 \mu \mathrm{L})$, and then transferred to the first vial. The reaction was stirred at room temperature. After 16 hours, $2 \mathrm{~mL}$ of $2 \mathrm{M}$ aqueous $\mathrm{Na}_{2} \mathrm{CO}_{3}$ was added and the mixture was extracted 4 times with $2 \mathrm{~mL}$ DCM. The combined organic layer was dried over anhydrous $\mathrm{Na}_{2} \mathrm{SO}_{4}$, solids were filtered, and the filtrate was concentrated by rotary evaporation. A crude ${ }^{1} \mathrm{H}$ NMR spectrum was collected with mesitylene as internal standard. The crude mixture was then purified by column chromatography.

Yield: $45 \%$; $1.2: 1 \mathrm{dr}$. Colorless oil. $\mathbf{R}_{\boldsymbol{f}}=0.20$ (hexane/EtOAc 1:1).

${ }^{1} \mathrm{H}$ NMR $\left(500 \mathrm{MHz}, \mathrm{CDCl}_{3}\right) \delta 5.84-5.75(\mathrm{~m}, 1 \mathrm{H}), 3.72-3.66(\mathrm{~m}, 1 \mathrm{H}), 3.42 \& 3.41(\mathrm{~s}, 3 \mathrm{H}), 2.86$ \& $2.83(\mathrm{dd}, \mathrm{J}=7.1,5.6 \mathrm{~Hz}, 1 \mathrm{H}), 2.71-2.60(\mathrm{~m}, 3 \mathrm{H}), 2.22 \& 2.21(\mathrm{~s}, 3 \mathrm{H}), 1.96-1.67(\mathrm{~m}, 4 \mathrm{H}), 1.57-$ $1.53(\mathrm{~m}, 1 \mathrm{H})$.

${ }^{13} \mathrm{C}$ NMR $\left(126 \mathrm{MHz}, \mathrm{CDCl}_{3}\right) \delta 206.2,206.1,169.9,169.9,120.4(q, \mathrm{~J}=277 \mathrm{~Hz}), 94.5,94.4,66.4(\mathrm{p}, \mathrm{J}$ $=35.3 \mathrm{~Hz}), 66.4(\mathrm{p}, \mathrm{J}=35.3 \mathrm{~Hz}), 57.1,57.0,48.8,48.7,35.2,31.7,31.7,31.3,31.3,30.6,30.6,28.4$, 27.2, 27.1 .

${ }^{19} \mathrm{~F}$ NMR $\left(471 \mathrm{MHz}, \mathrm{CDCl}_{3}\right) \delta-73.3$.

IR (neat, $\mathrm{cm}^{-1}$ ) v 2927, 1780, 1716, 1358, 1287, 1227, 1198, 1108, 906, 692.

HRMS (ASAP) $\mathrm{m} / \mathrm{z}\left[\mathrm{C}_{13} \mathrm{H}_{18} \mathrm{~F}_{6} \mathrm{NO}_{4}\right]^{+}\left([\mathrm{M}+\mathrm{H}]^{+}\right)$calculated 366.1135, found 366.1140 . 


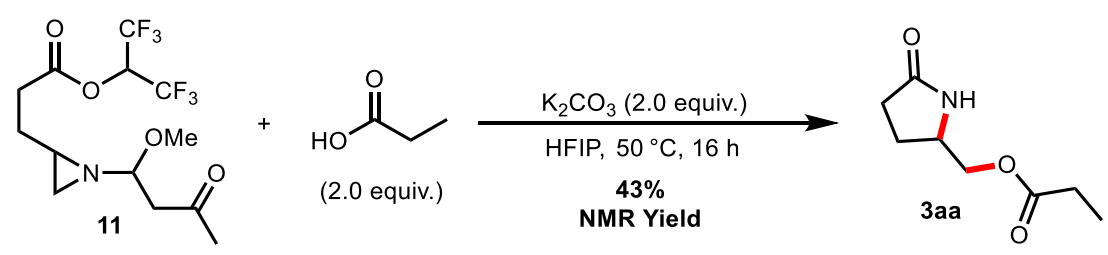

10 (8.6 mg, $0.024 \mathrm{mmol})$, propionic acid $(4.5 \mu \mathrm{L}, 0.06 \mathrm{mmol}), \mathrm{K}_{2} \mathrm{CO}_{3}(0.7 \mathrm{mg}, 0.005 \mathrm{mmol})$ and HFIP $(80 \mu \mathrm{L})$ were combined in a $1 \mathrm{dram}$ vial with a magnetic stir bar. The mixture was stirred at $50{ }^{\circ} \mathrm{C}$. After 16 hours, $2 \mathrm{~mL}$ of $2 \mathrm{M}$ aqueous $\mathrm{Na}_{2} \mathrm{CO}_{3}$ was added and the mixture was extracted 4 times with $2 \mathrm{~mL} \mathrm{DCM}$. The combined organic layer was dried over anhydrous $\mathrm{Na}_{2} \mathrm{SO}_{4}$, solids were filtered, and the filtrate was concentrated by rotary evaporation. A crude ${ }^{1} \mathrm{H}$ NMR spectrum was collected with mesitylene as internal standard.

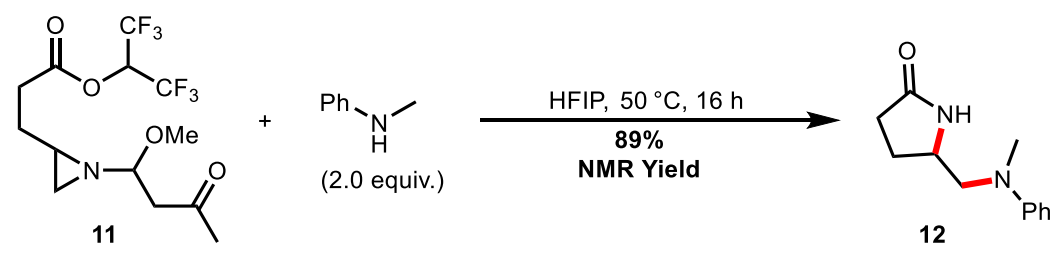

$10(9.1 \mathrm{mg}, 0.025 \mathrm{mmol}), \mathrm{N}$-methyl aniline $(5.4 \mu \mathrm{L}, 0.05 \mathrm{mmol})$ and HFIP $(100 \mu \mathrm{L})$ were combined in a 1 dram vial with a magnetic stir bar. The mixture was stirred at $50{ }^{\circ} \mathrm{C}$. After 16 hours, $2 \mathrm{~mL}$ of $2 \mathrm{M}$ aqueous $\mathrm{Na}_{2} \mathrm{CO}_{3}$ was added and the mixture was extracted 4 times with $2 \mathrm{~mL} \mathrm{DCM}$. The combined organic layer was dried over anhydrous $\mathrm{Na}_{2} \mathrm{SO}_{4}$, solids were filtered, and the filtrate was concentrated by rotary evaporation. A crude ${ }^{1} \mathrm{H}$ NMR spectrum was collected with mesitylene as internal standard.
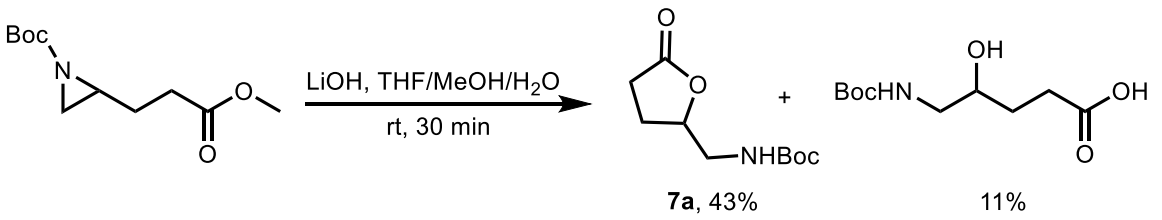

$t$-Butyl 2-(3-methoxy-3-oxopropyl)aziridine-1-carboxylate $(83 \mathrm{mg}, 0.36 \mathrm{mmol}$ ) (prepared according to a literature report ${ }^{7}$ ) was dissolved in THF/MeOH $(5 \mathrm{~mL} / 1 \mathrm{~mL}$ ). A solution of LiOH (26 $\mathrm{mg}, 1.1 \mathrm{mmol})$ in $\mathrm{H}_{2} \mathrm{O}(1 \mathrm{~mL})$ was added at room temperature, and the mixture was stirred for 30 min. The reaction was quenched by adding $1 \mathrm{M} \mathrm{HCl}$ solution, which made $\mathrm{pH}$ 2-3. The mixture was then extracted three times with EtOAc, and the combined organic layers were dried over anhydrous $\mathrm{Na}_{2} \mathrm{SO}_{4}$, filtered and concentrated under vacuum. The crude mixture was further purified through column chromatography to give $32.4 \mathrm{mg}$ of lactone $7 \mathrm{a}$ and $8.8 \mathrm{mg}$ of corresponding hydrolysis by-product.

7a: ${ }^{1} \mathrm{H}$ NMR $\left(500 \mathrm{MHz}, \mathrm{CDCl}_{3}\right.$ ) $\delta 4.96(\mathrm{~s}, 1 \mathrm{H}), 4.61$ (qd, J = 7.1, 3.3 Hz, $1 \mathrm{H}$ ), 3.53 (ddd, J = 14.8, 6.9, $3.3 \mathrm{~Hz}, 1 \mathrm{H}$ ), $3.28(\mathrm{dt}, \mathrm{J}=14.7,6.2 \mathrm{~Hz}, 1 \mathrm{H}), 2.56(\mathrm{dd}, \mathrm{J}=9.5,7.2 \mathrm{~Hz}, 2 \mathrm{H}$ ), 2.30 (dq, J = 13.2, 7.1 Hz, $1 \mathrm{H}), 1.98(\mathrm{dtd}, \mathrm{J}=13.0,9.5,7.8 \mathrm{~Hz}, 1 \mathrm{H}), 1.45(\mathrm{~s}, 9 \mathrm{H})$.

Acid: ${ }^{1} \mathrm{H}$ NMR $\left(500 \mathrm{MHz}, \mathrm{CDCl}_{3}\right) \delta 5.06(\mathrm{~s}, 1 \mathrm{H}), 3.83-3.73(\mathrm{~m}, 1 \mathrm{H}), 3.36-3.23(\mathrm{~m}, 1 \mathrm{H}), 3.15-$ $3.05(\mathrm{~m}, 1 \mathrm{H}), 2.56(\mathrm{t}, J=7.0 \mathrm{~Hz}, 2 \mathrm{H}), 1.86-1.72(\mathrm{~m}, 2 \mathrm{H}), 1.47(\mathrm{~s}, 9 \mathrm{H})$. 


\section{X-Ray Crystal Structure}

Single crystal X-ray diffraction was performed at the Shared Materials Characterization Laboratory at Columbia University. Use of the SMCL was made possible by funding from Columbia University.

Single crystal X-ray diffraction. Data for compound 5-OH-3dg, 3ej and 3gh was collected on an Agilent SuperNova diffractometer using mirror-monochromated $\mathrm{Cu} \mathrm{K} \alpha$ or Mo K $\alpha$ radiation. Data collection, integration, scaling (ABSPACK) and absorption correction (face-indexed Gaussian integration or numeric analytical methods) were performed in CrysAlisPro. Structure solution was performed using SheIXT. Subsequent refinement was performed by full-matrix least-squares on $\mathrm{F}^{2}$ in ShelXL. Olex2 was used for viewing and to prepare CIF files. ORTEP graphics were prepared in CrystalMaker. Thermal ellipsoids are rendered at the $50 \%$ probability level.

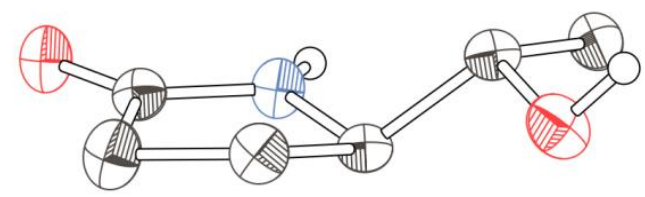

Molecular structure of 5-OH-3dg (crystallized from $\mathrm{CHCl}_{3} /$ pentane).

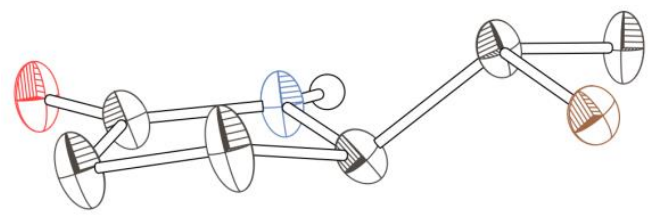

Molecular structure of $3 e j$ (crystallized from $\mathrm{C}_{6} \mathrm{H}_{6} /$ pentane).

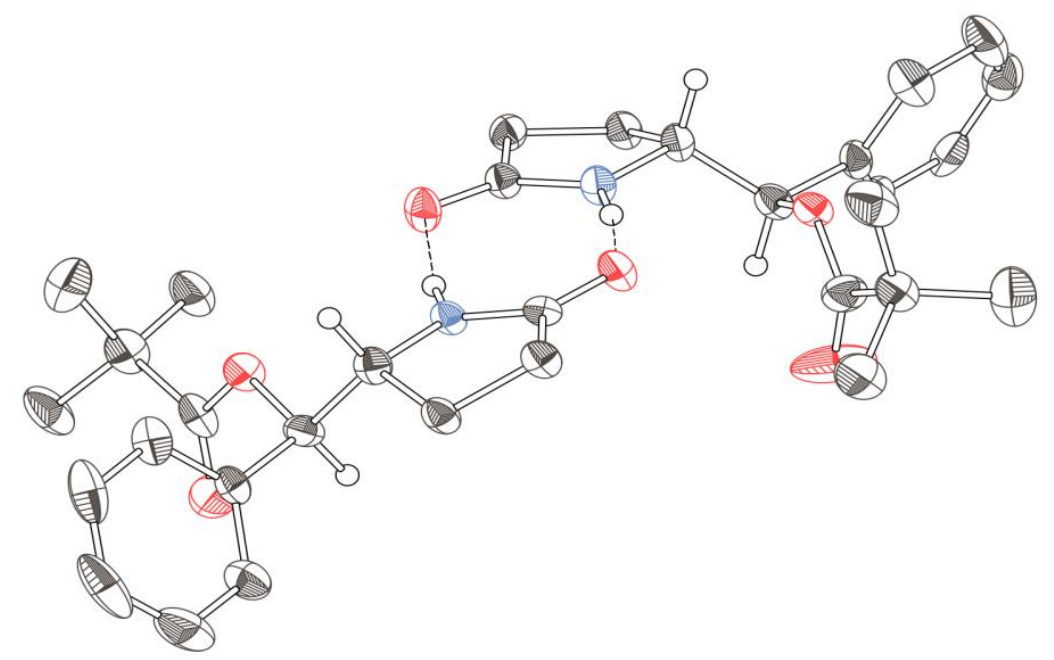

Molecular structure of $3 g h$ (crystallized from $\mathrm{C}_{6} \mathrm{H}_{6} /$ pentane). 


\begin{tabular}{|c|c|c|c|}
\hline Compound & 5-OH-3dg & 3ej & 3gh \\
\hline Formula & $\mathrm{C}_{6} \mathrm{H}_{11} \mathrm{NO}_{2}$ & $\mathrm{C}_{6} \mathrm{H}_{10} \mathrm{NOBr}$ & $\mathrm{C}_{16} \mathrm{H}_{21} \mathrm{NO}_{3}$ \\
\hline MW & 129.16 & 192.06 & 275.34 \\
\hline Space group & $\mathrm{P} 2{ }_{1} / \mathrm{n}$ & $P-1$ & $22.1740(10)$ \\
\hline$a(\AA)$ & $4.57340(17)$ & $5.9616(5)$ & $10.4504(4)$ \\
\hline$b(\AA)$ & $9.8222(4)$ & $7.0596(5)$ & $28.6661(14)$ \\
\hline$c(\AA ̊)$ & $15.0672(6)$ & $9.5102(6)$ & 90 \\
\hline$\alpha\left({ }^{\circ}\right)$ & 90 & $70.172(6)$ & $111.129(5)$ \\
\hline $6\left(^{\circ}\right)$ & $94.519(4)$ & $89.251(6)$ & 90 \\
\hline$\nu\left(^{\circ}\right)$ & 90 & $79.122(7)$ & $6196.1(5)$ \\
\hline$V\left(\AA^{3}\right)$ & $674.72(5)$ & $369.19(5)$ & 16 \\
\hline Z & 4 & 2 & 1.181 \\
\hline$\rho_{\text {calc }}\left(\mathrm{g} \mathrm{cm}^{-3}\right)$ & 1.271 & 1.728 & 1.181 \\
\hline $\mathrm{T}(\mathrm{K})$ & 100 & 100 & 100 \\
\hline$\lambda(\AA ̊)$ & 1.54184 & 0.71073 & 0.71073 \\
\hline $2 \theta_{\min }, 2 \theta_{\max }$ & 11,145 & 8,59 & 7,59 \\
\hline Nref & 5116 & 2644 & 30794 \\
\hline$R($ int $), R(\sigma)$ & $.0298, .0245$ & $.0347, .0655$ & $.0552, .0798$ \\
\hline$\mu\left(\mathrm{mm}^{-1}\right)$ & 0.787 & 5.486 & 0.081 \\
\hline \multirow{2}{*}{ Size $(\mathrm{mm})$} & $.27 \times .12$ & $.56 \times .24$ & $.46 \times .24$ \\
\hline & $x .07$ & $\mathrm{x} .18$ & $x .12$ \\
\hline$T_{\max } / T_{\min }$ & 1.08 & 7.81 & 1.84 \\
\hline Data & 1338 & 1649 & 13581 \\
\hline Restraints & 12 & 77 & 239 \\
\hline Parameters & 107 & 125 & 842 \\
\hline $\mathbf{R}_{1}$ (obs) & 0.0478 & 0.0323 & 0.0673 \\
\hline$w_{2}$ (all) & 0.1358 & 0.0647 & 0.1432 \\
\hline S & 1.077 & 0.993 & 1.028 \\
\hline $\begin{array}{r}\text { Peak, hole }\left(\mathrm{e}^{-}\right. \\
\left.\AA^{-3}\right)\end{array}$ & $0.23,-0.19$ & $0.54,-0.57$ & $0.41,-0.21$ \\
\hline
\end{tabular}

\section{Reference}

1. Conway, J. H.; Rovis, T. J. Am. Chem. Soc. 2018, 140, 135.

2. Wu, F.; Stewart, S.; Ariyarathna, J. P.; Li, W. ACS Catal. 2018, 8, 1921.

3. Shuler, S. A.; Yin, G.; Krause, S. B.; Vesper, C. M.; Watson, D. A. J. Am. Chem. Soc. 2016, 138, 13830.

4. Sustmann, R.; Hopp, P.; Holl, P. Tetrahedron Lett.1989, 30, 689.

5. Braddock,D. C.; Cansella, G.; Hermitageb, S. A. Chem. Commun. 2006, 2483.

6. Liu, M.; Yang, P.; Karunananda, M. K.; Wang, Y.; Liu, P.; Engle, K. M. J. Am. Chem. Soc.2018, 140, 175805.

7. Park, J.; Tian, G. R.; Kim, D. H. J. Org. Chem. 2001, 66, 3696. 
10. NMR Spectra

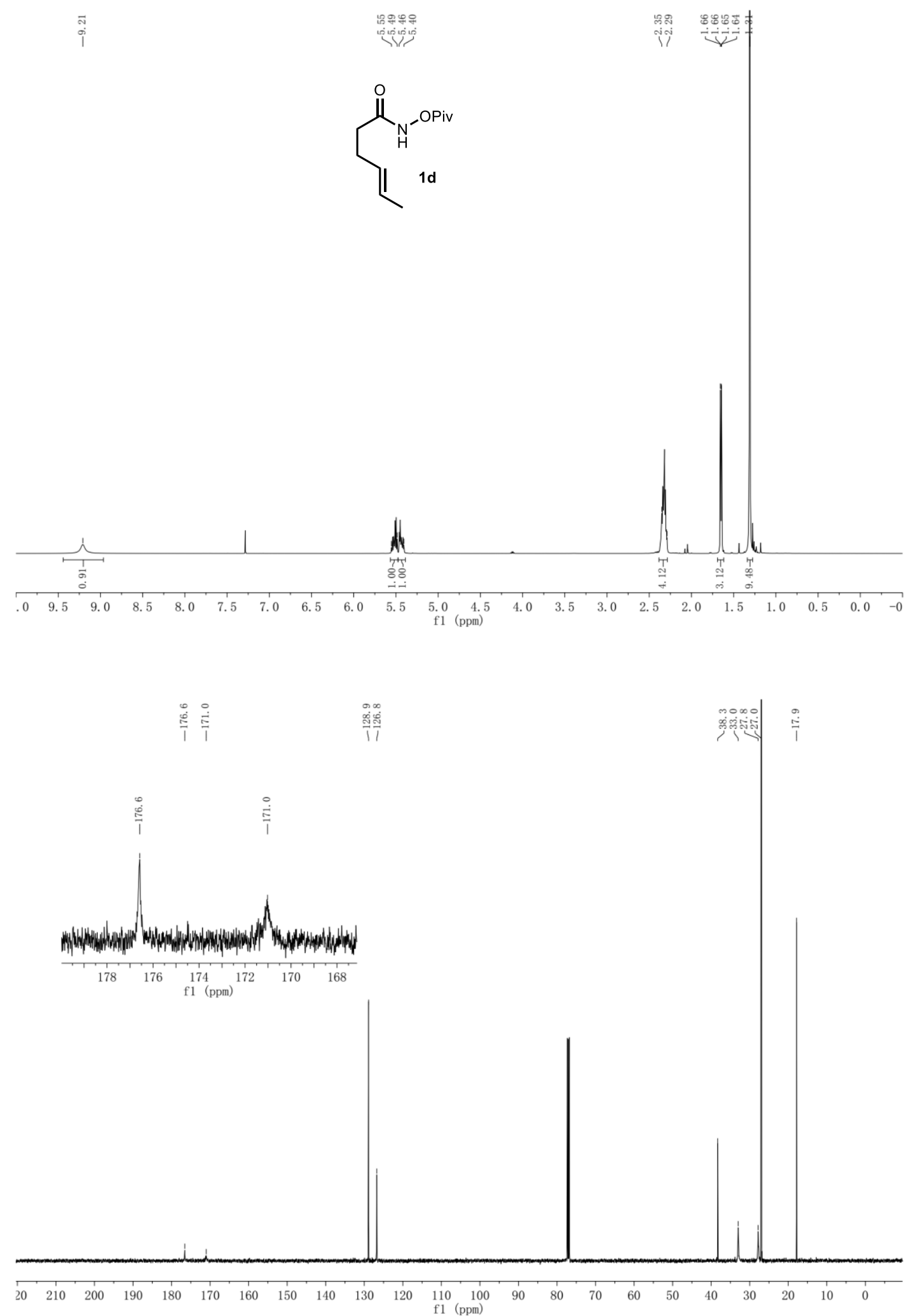




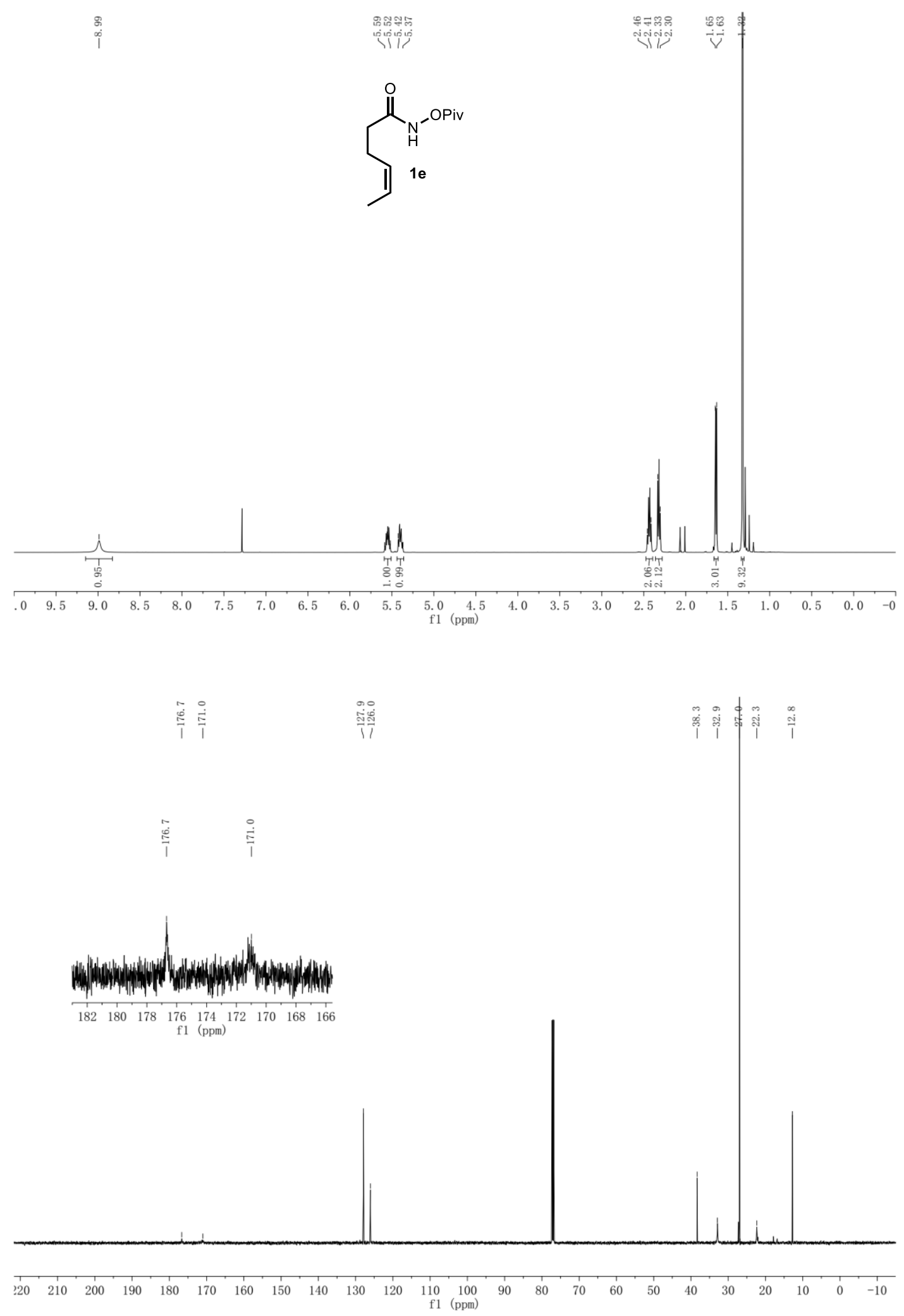



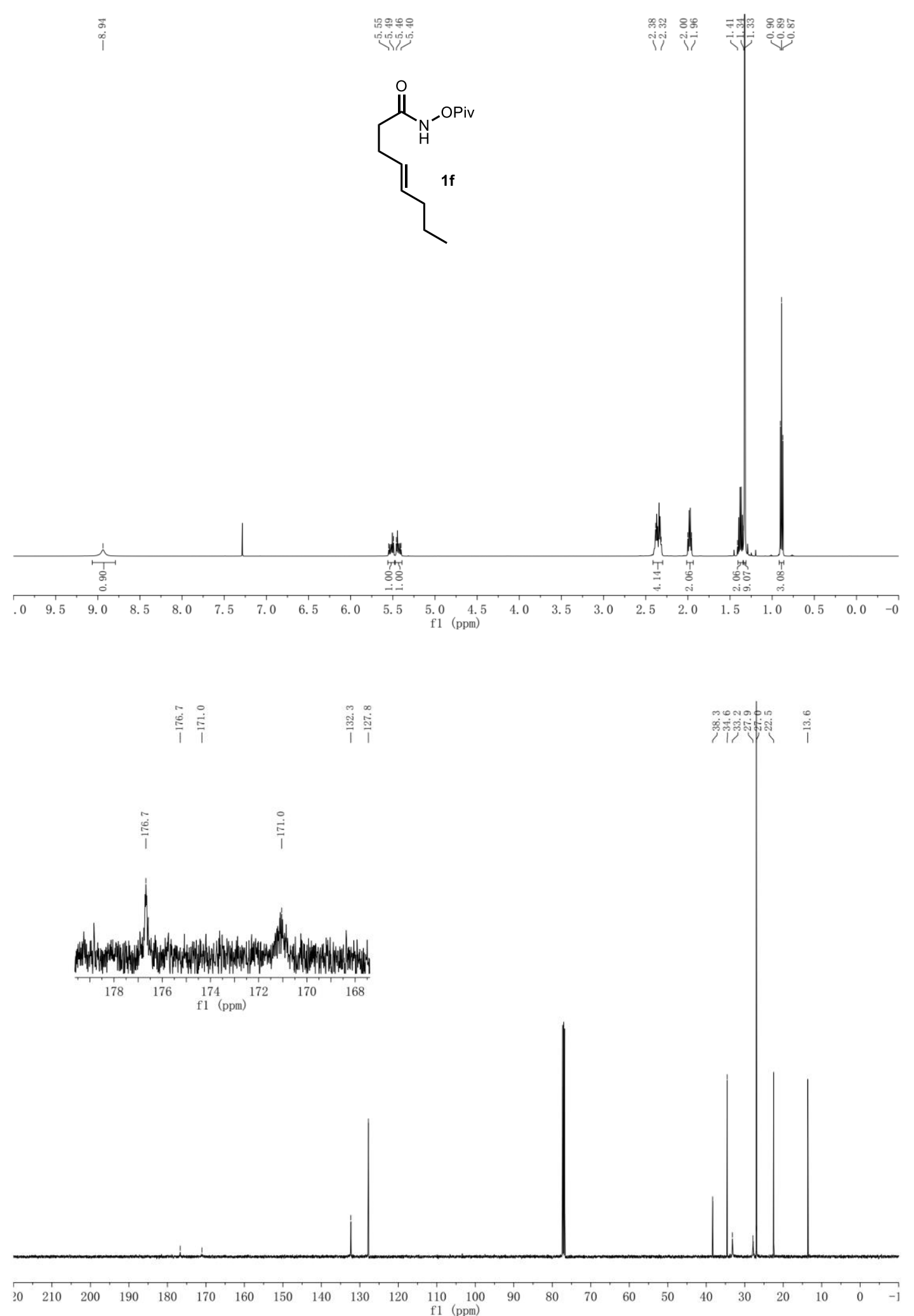

S27 
$\underset{\substack{0 \\ 0}}{0}$

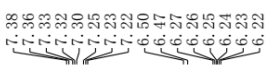

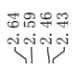
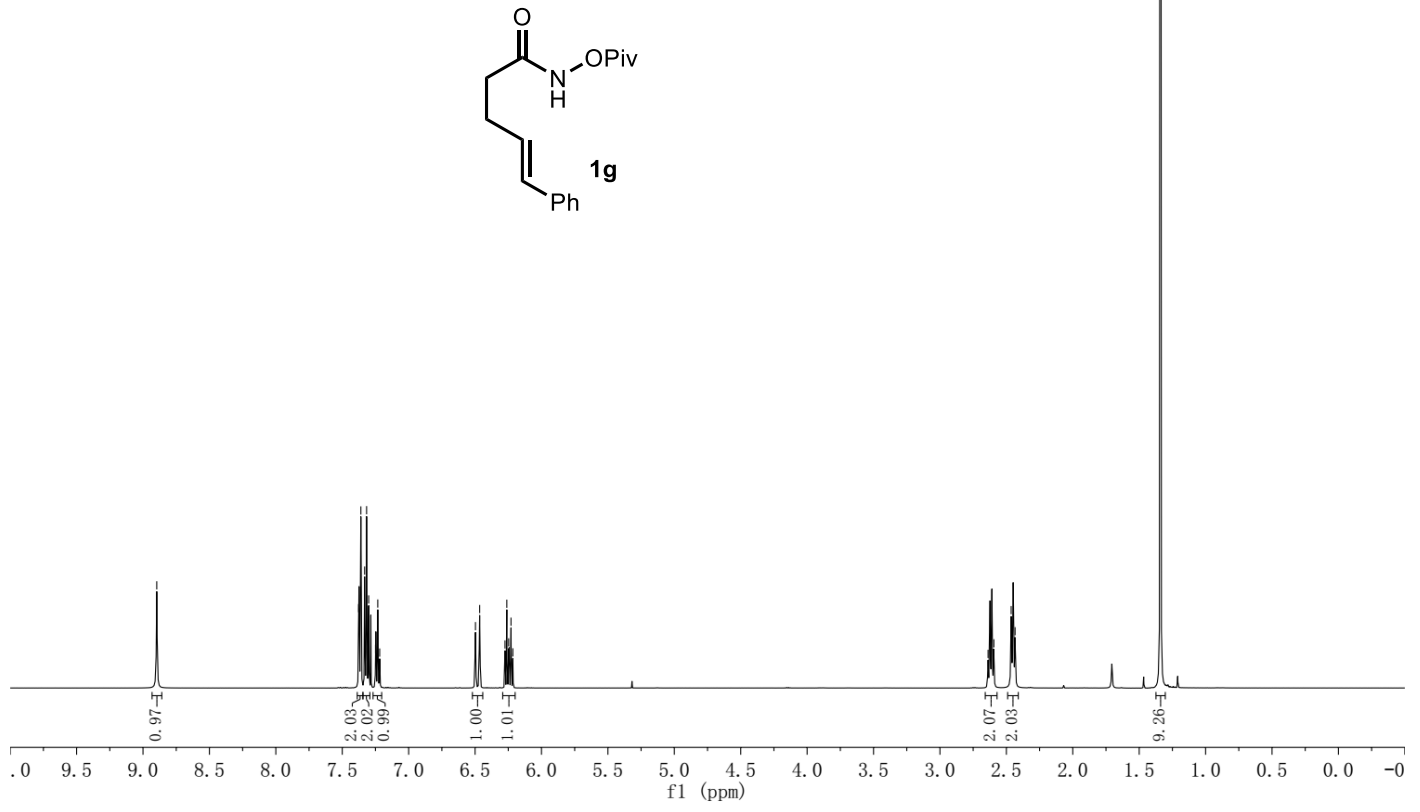

它

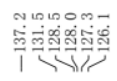

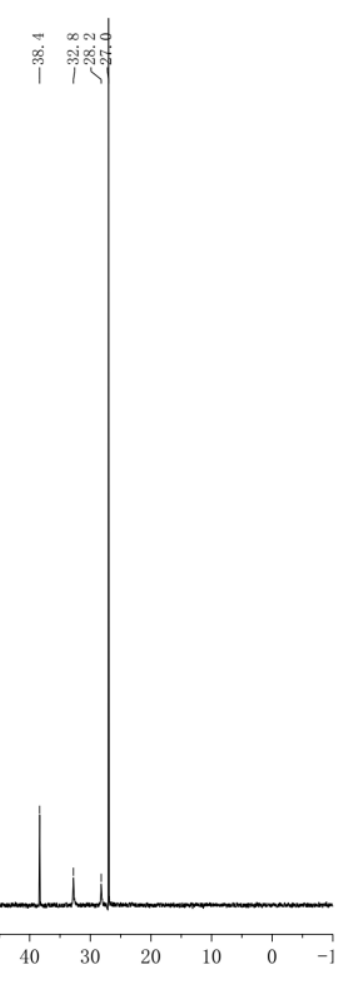

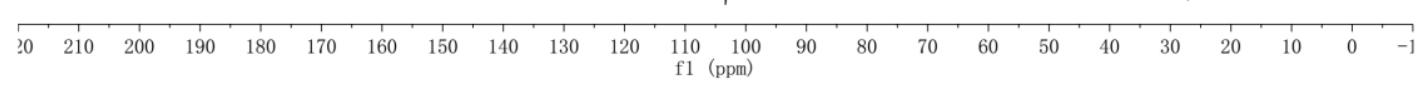

S28 


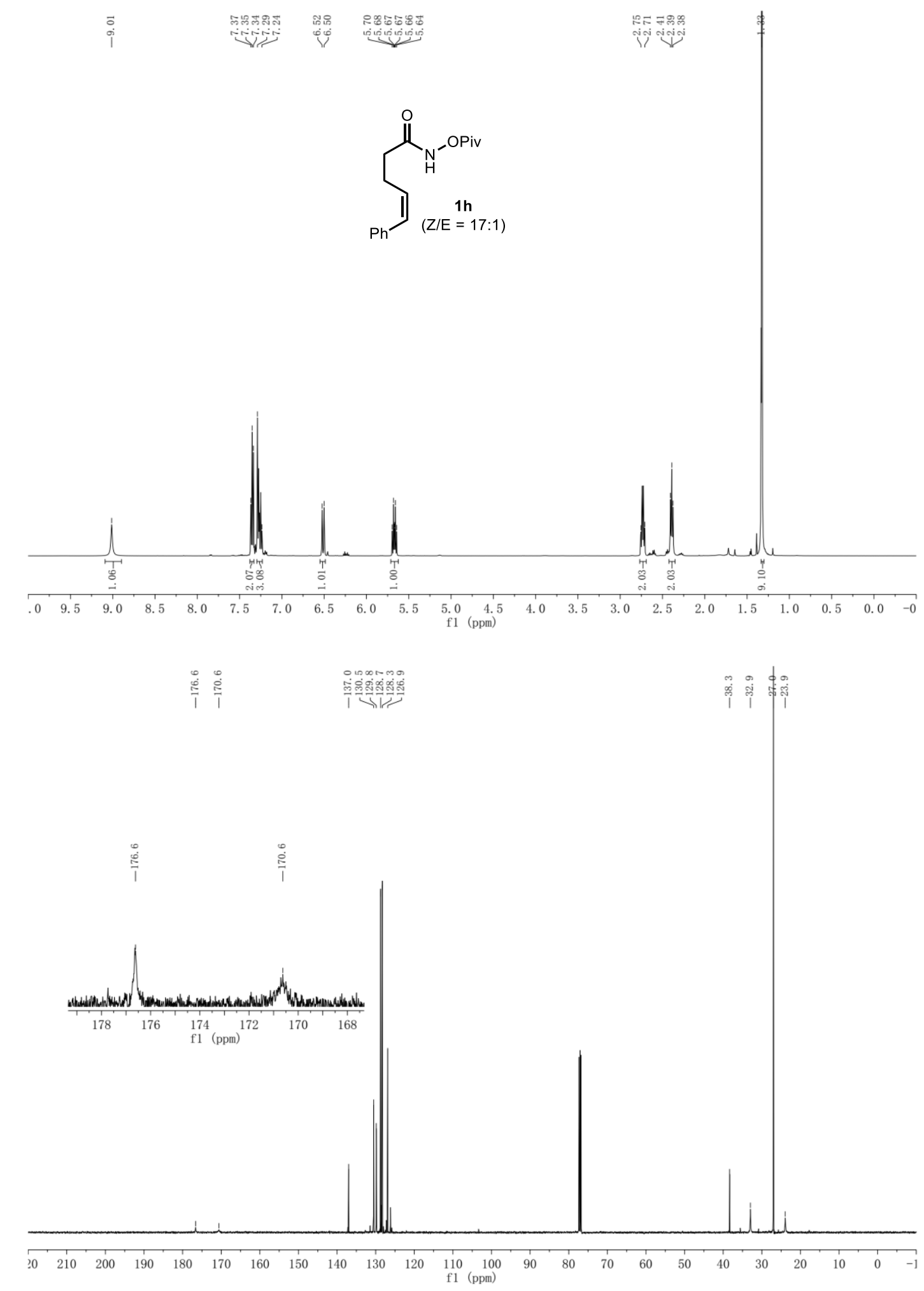




ivi

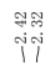
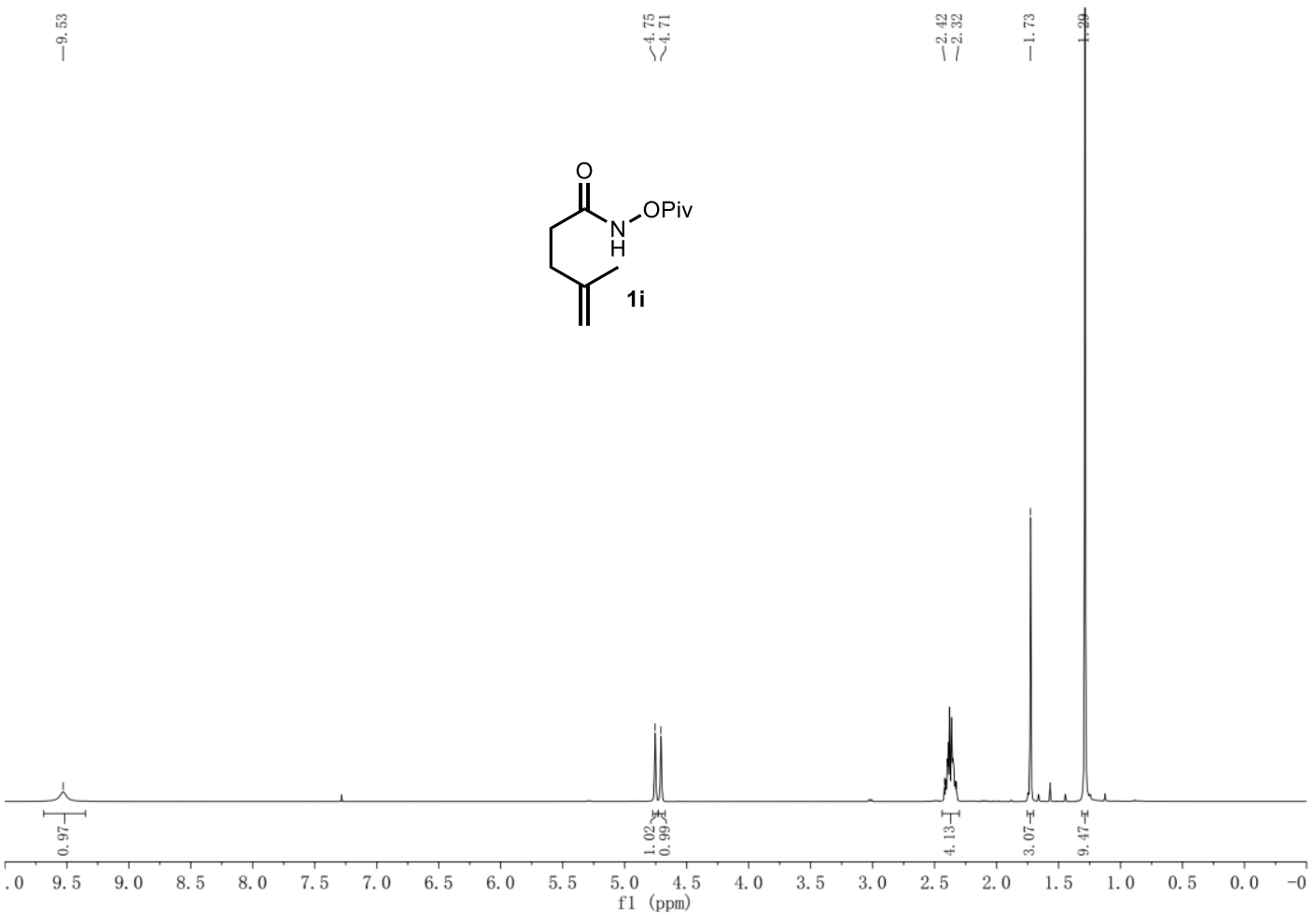

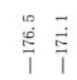

$\stackrel{0}{1}$

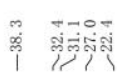
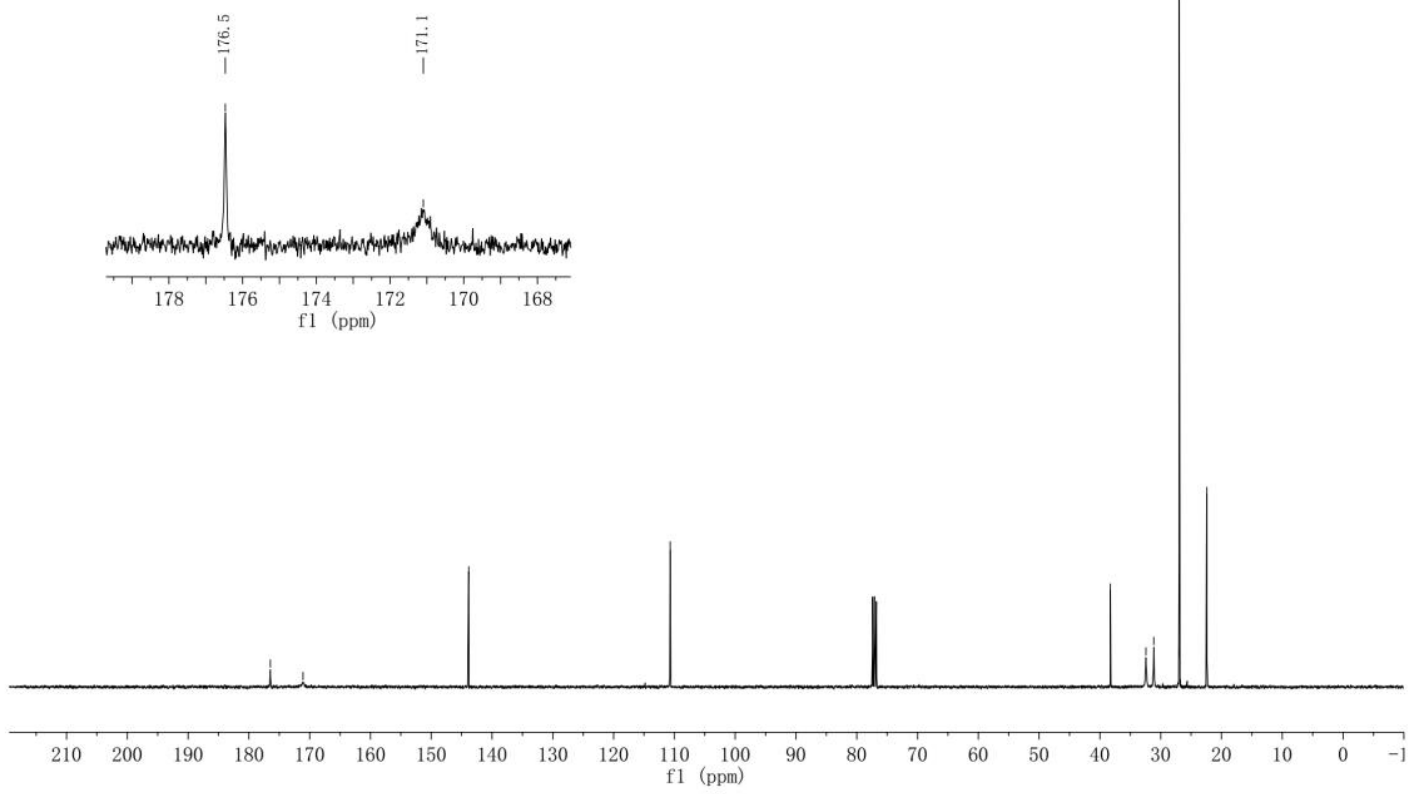

S30 


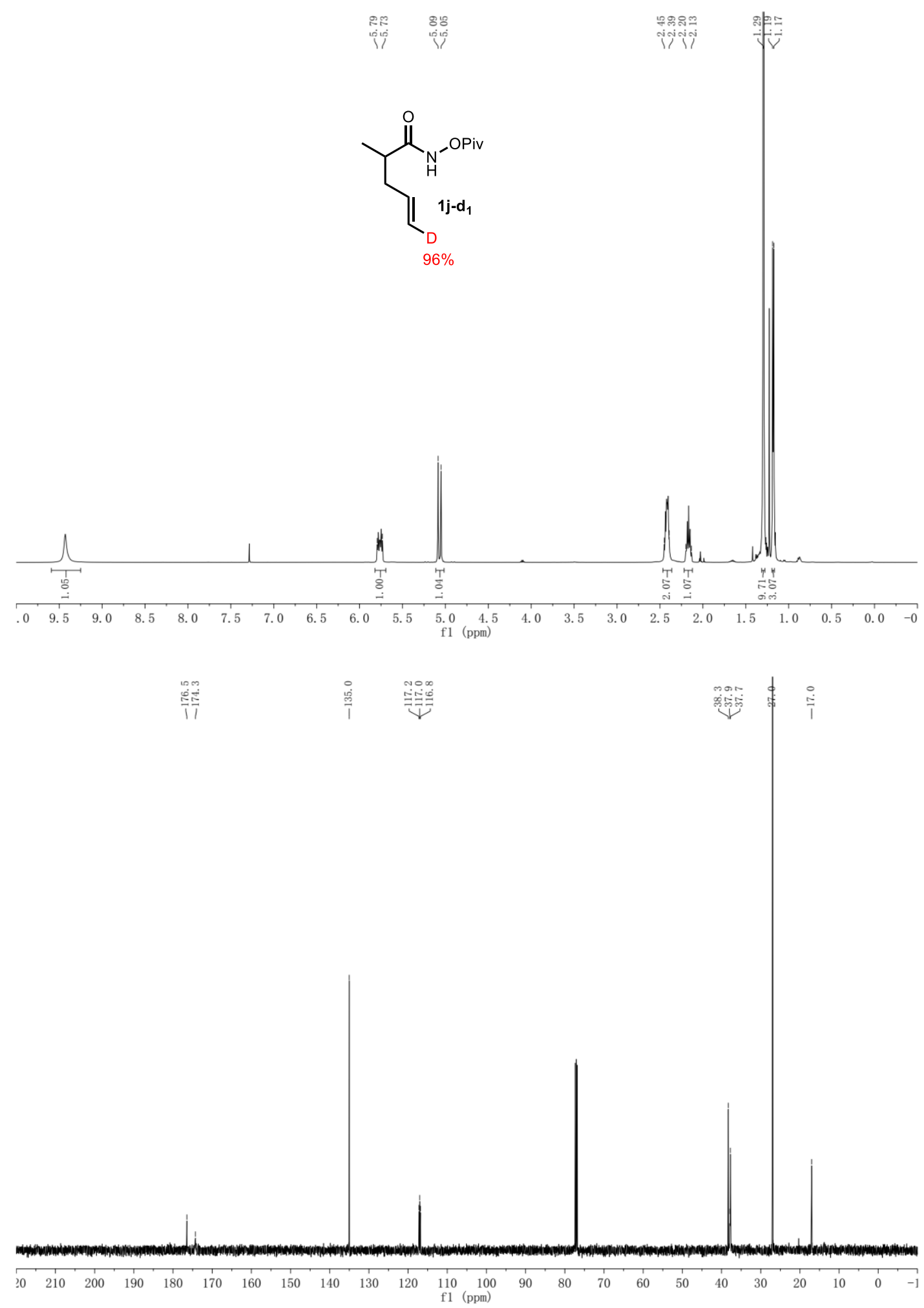



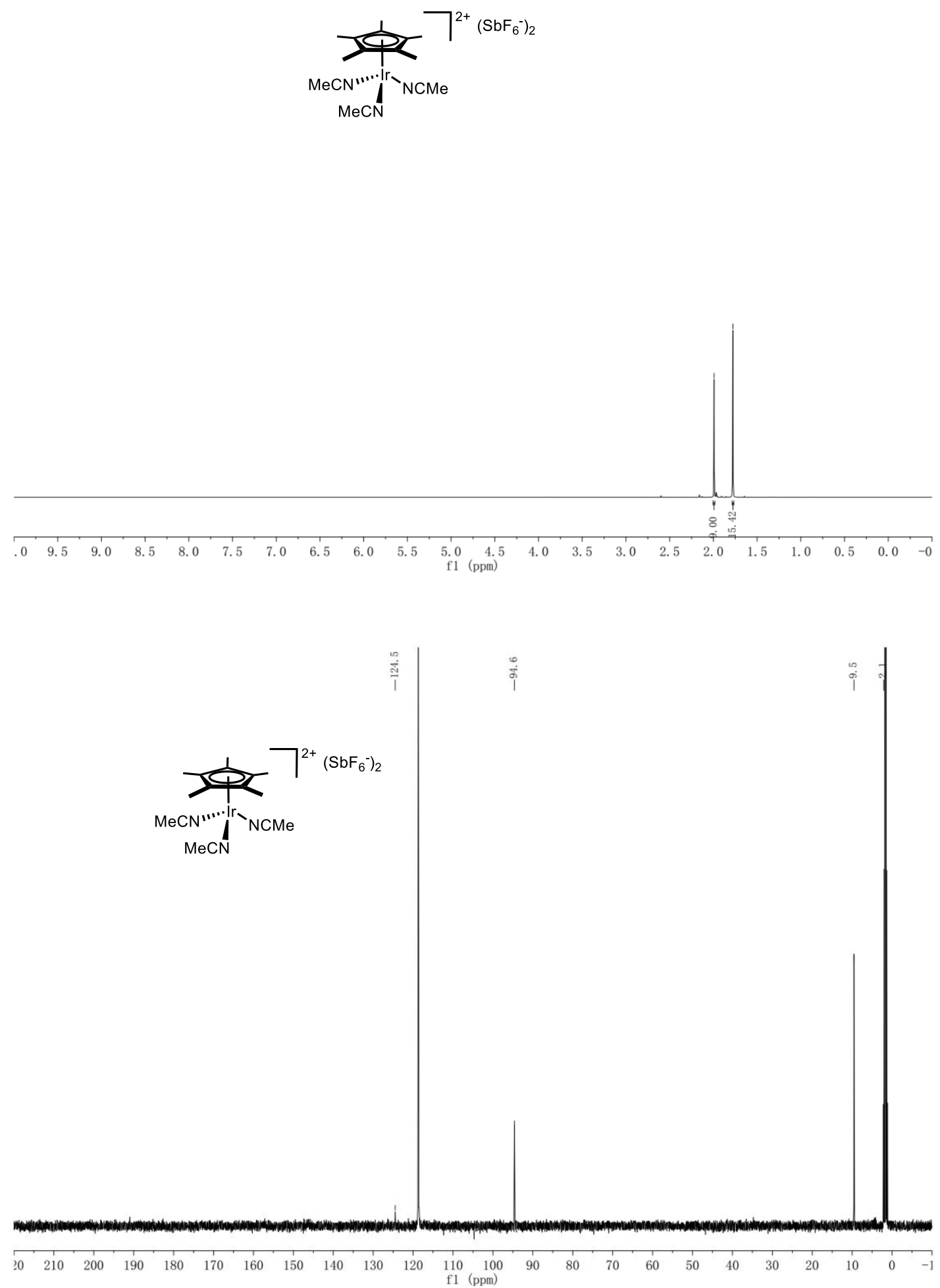

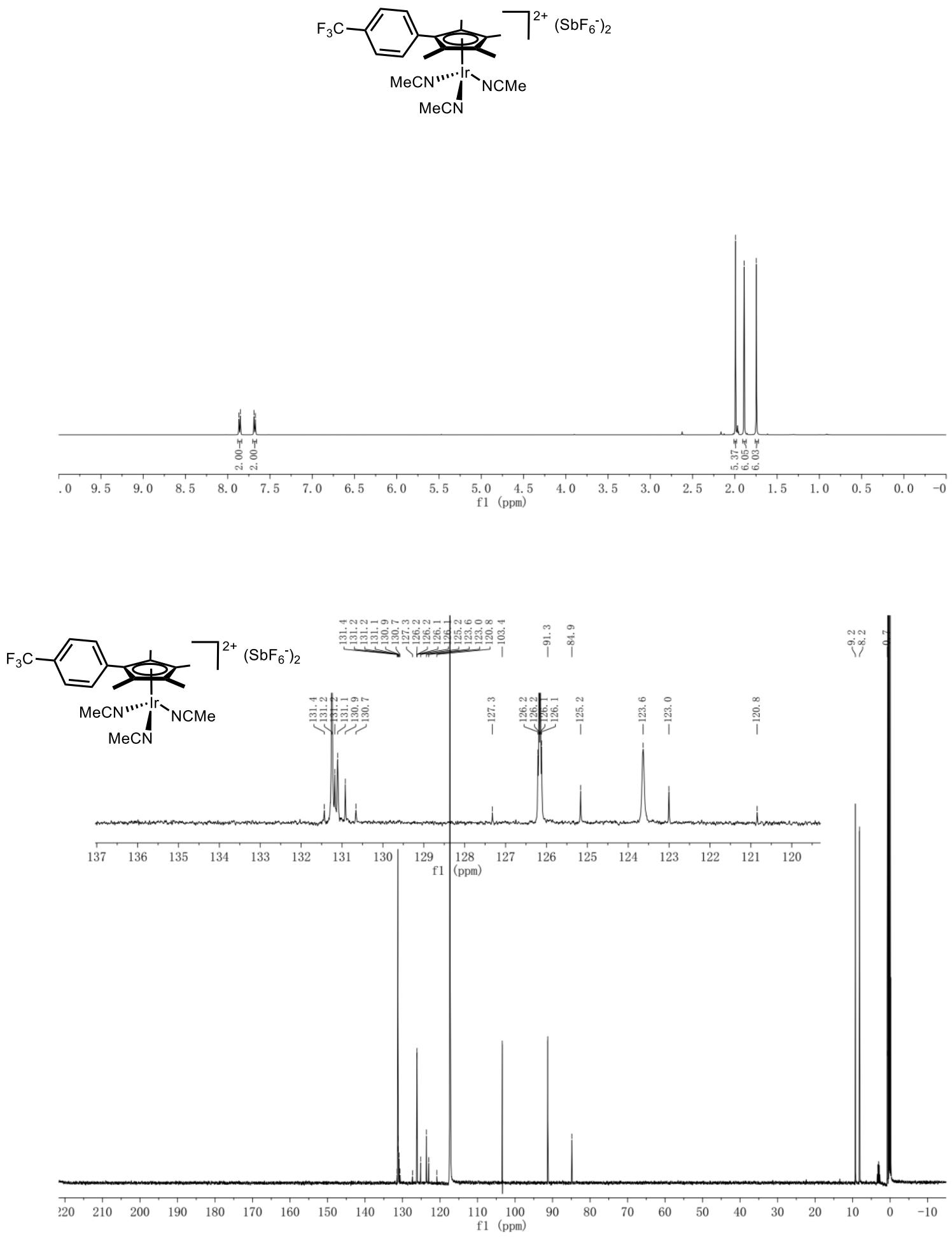
${ }^{19} \mathrm{~F}$ NMR

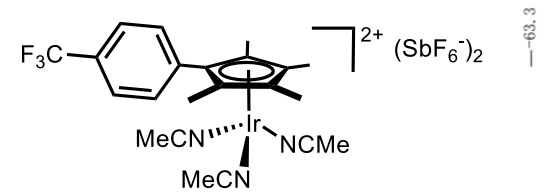

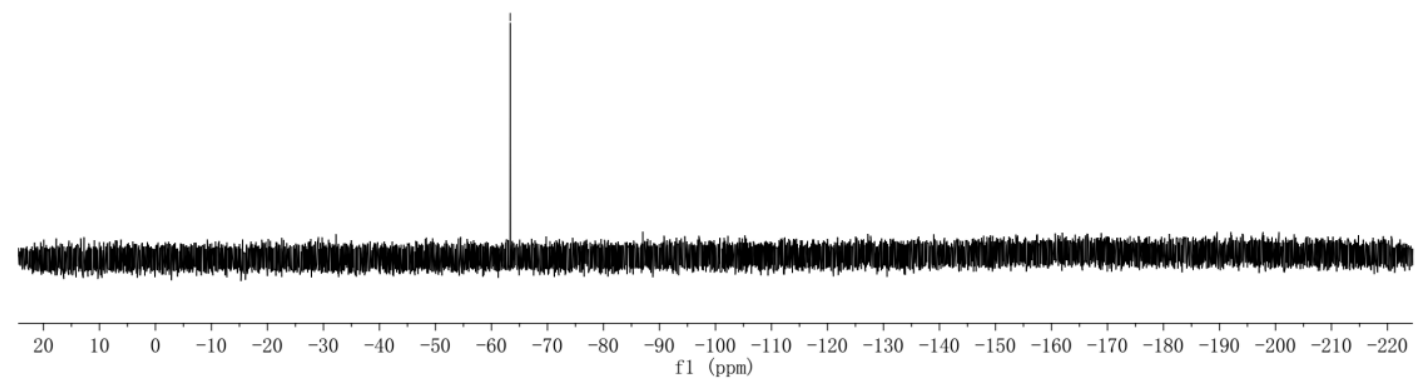


<smiles>CCC(=O)OCC1CCC(=O)N1</smiles>

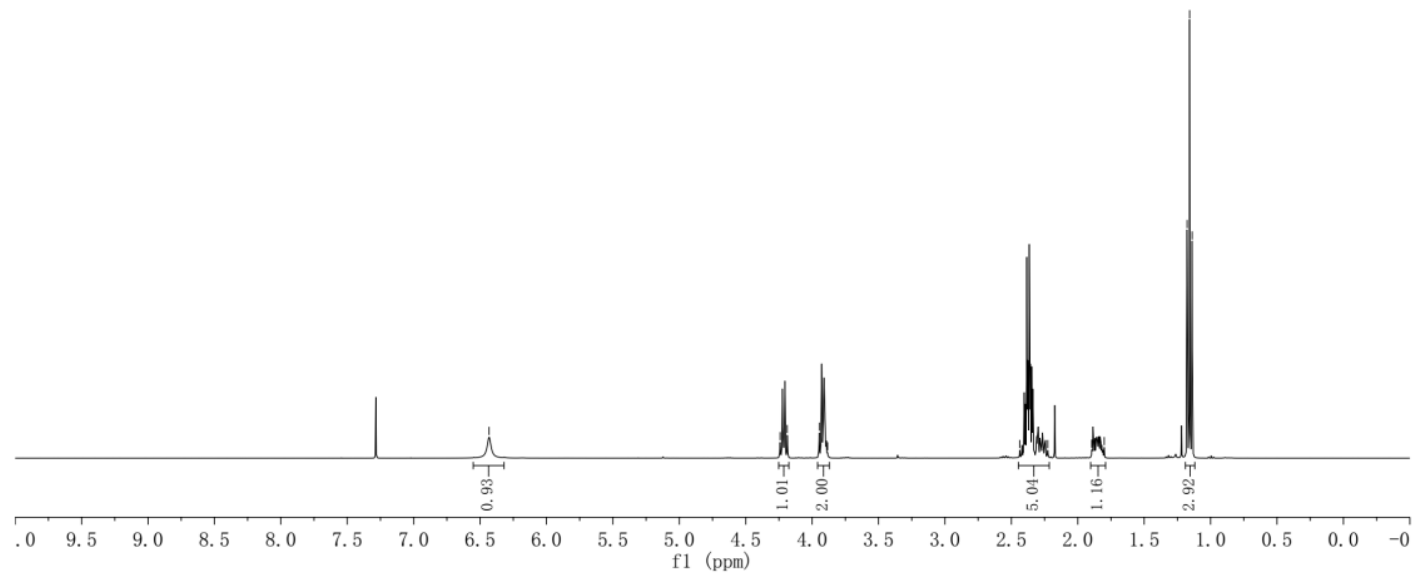




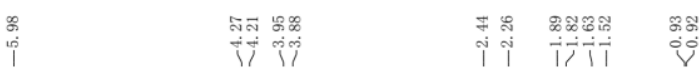
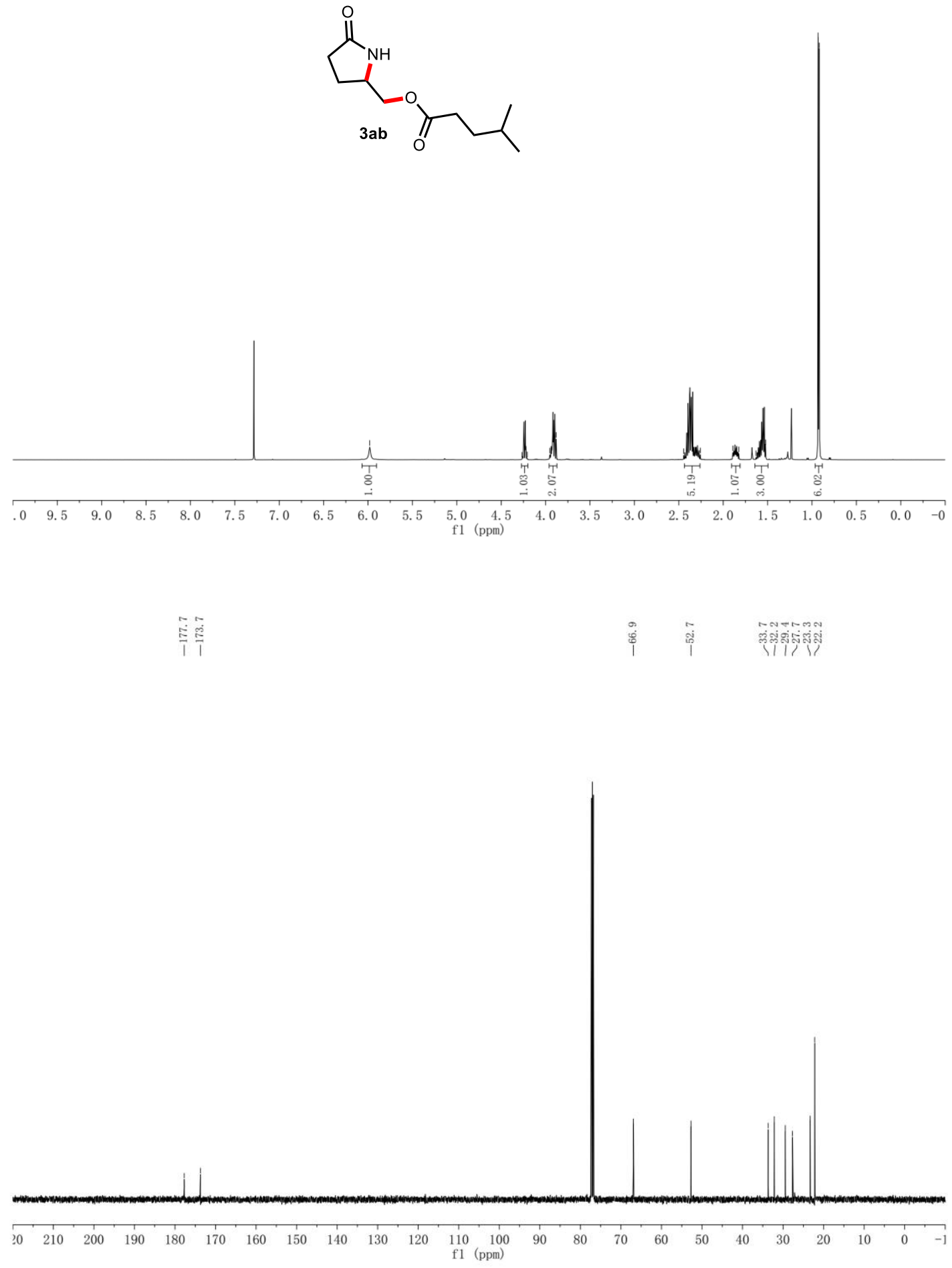


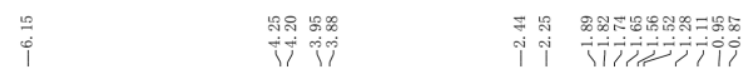
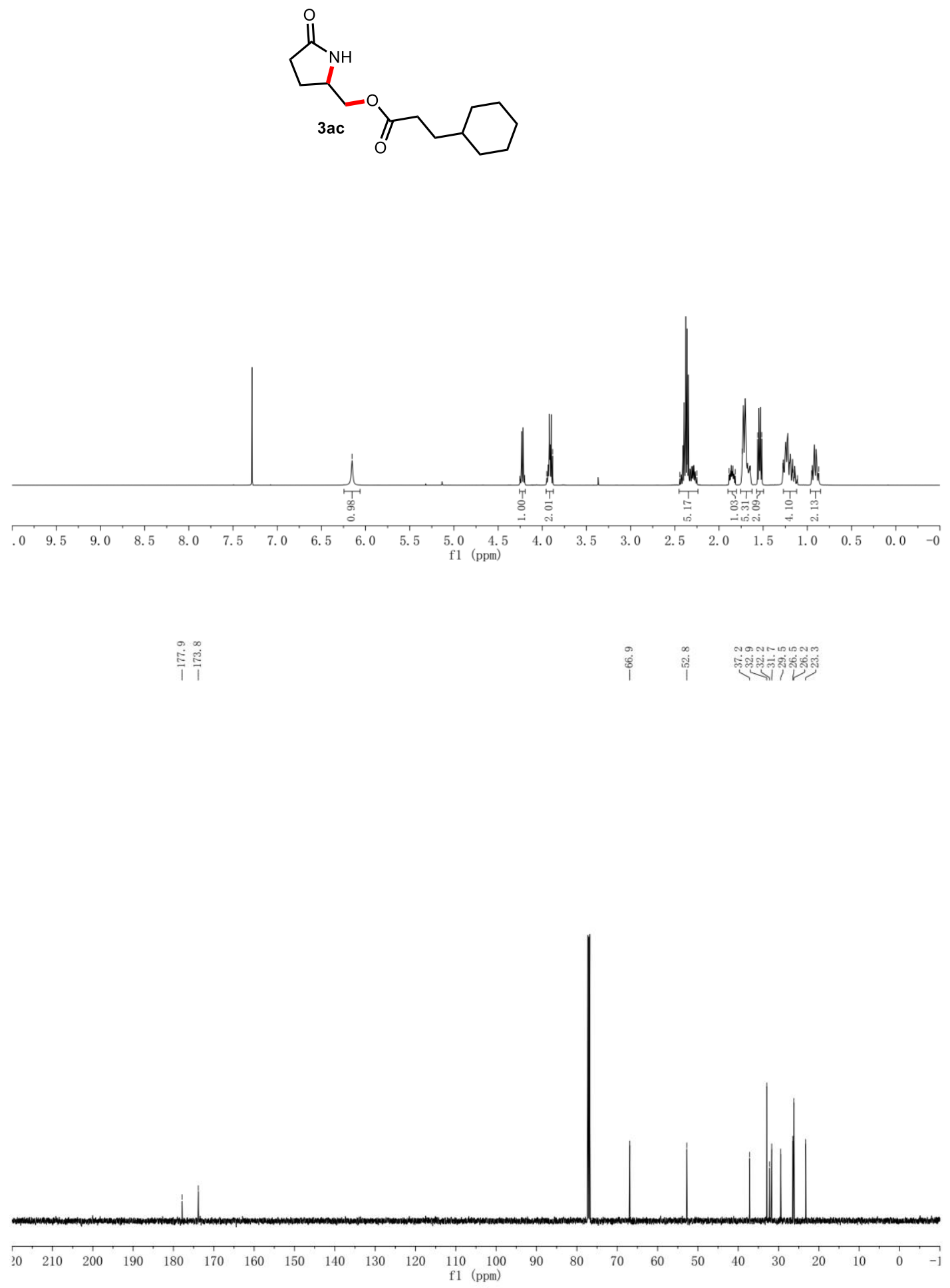


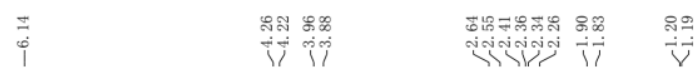<smiles>CC(C)C(=O)OC[C@H]1CCC(=O)N1</smiles>

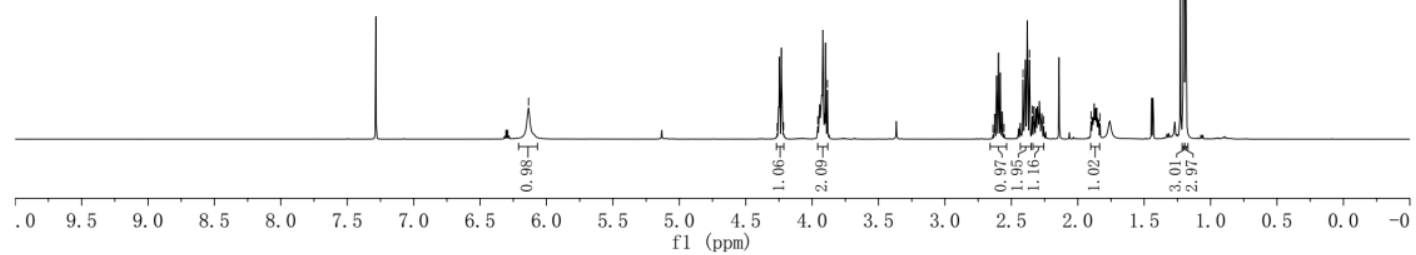

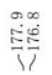

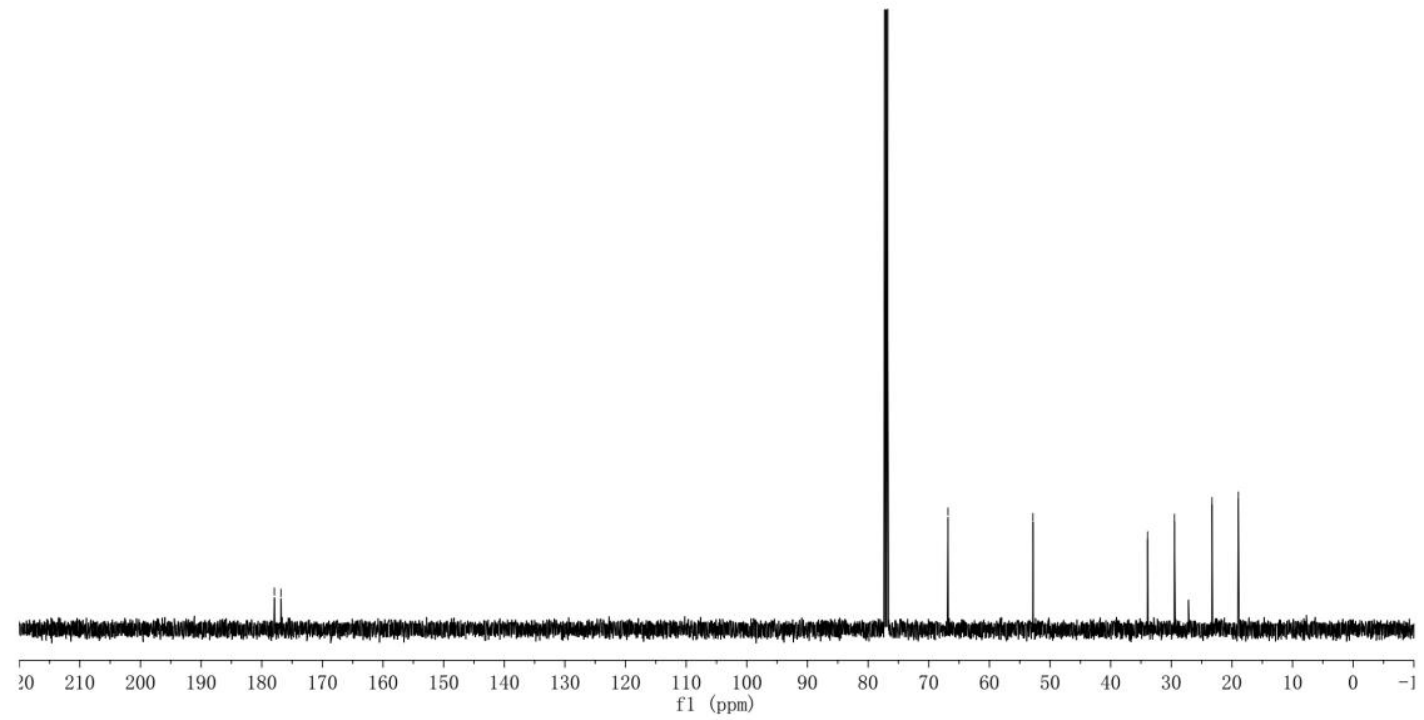



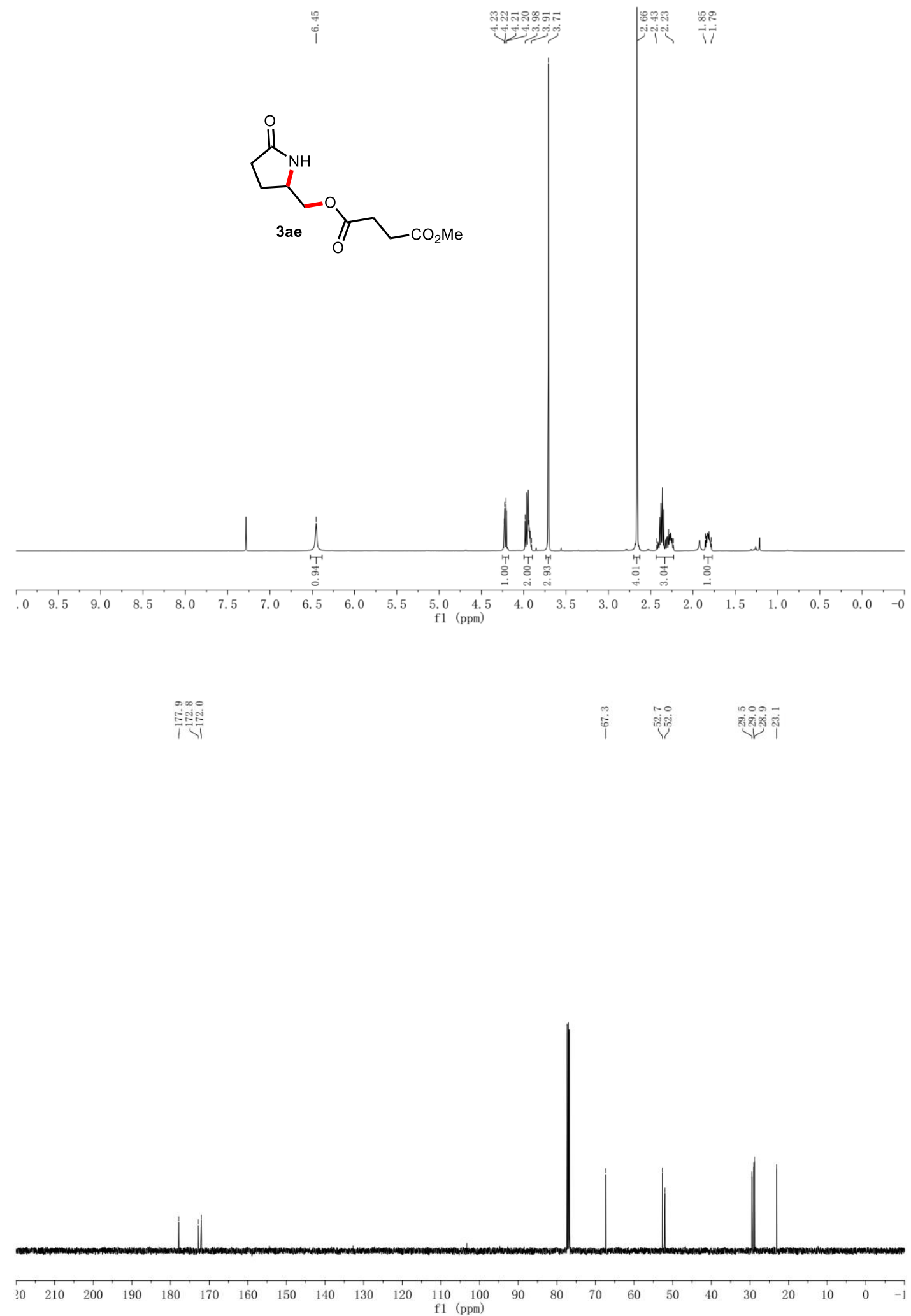

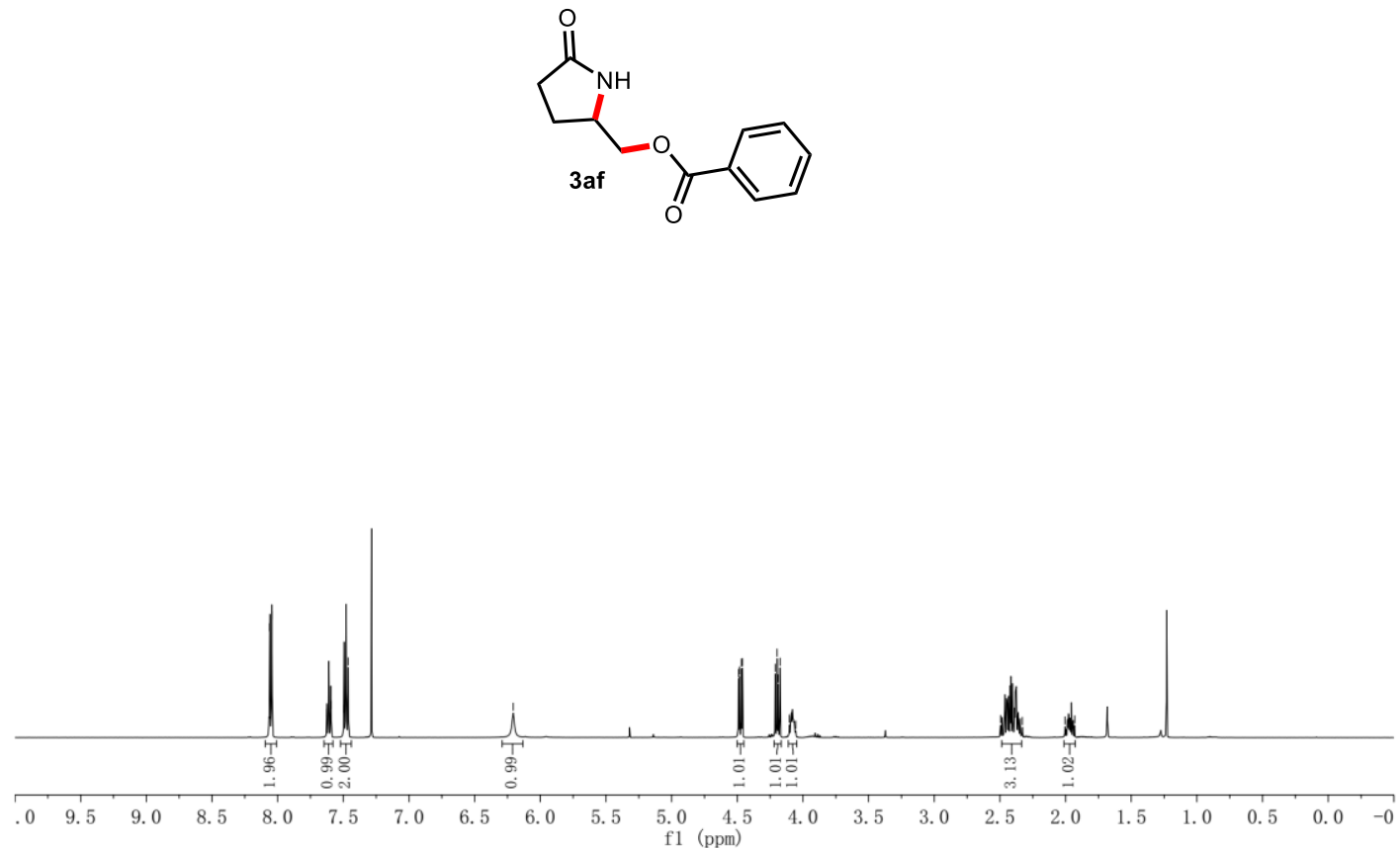

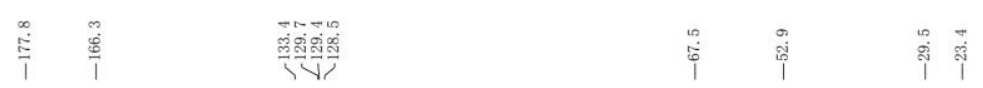

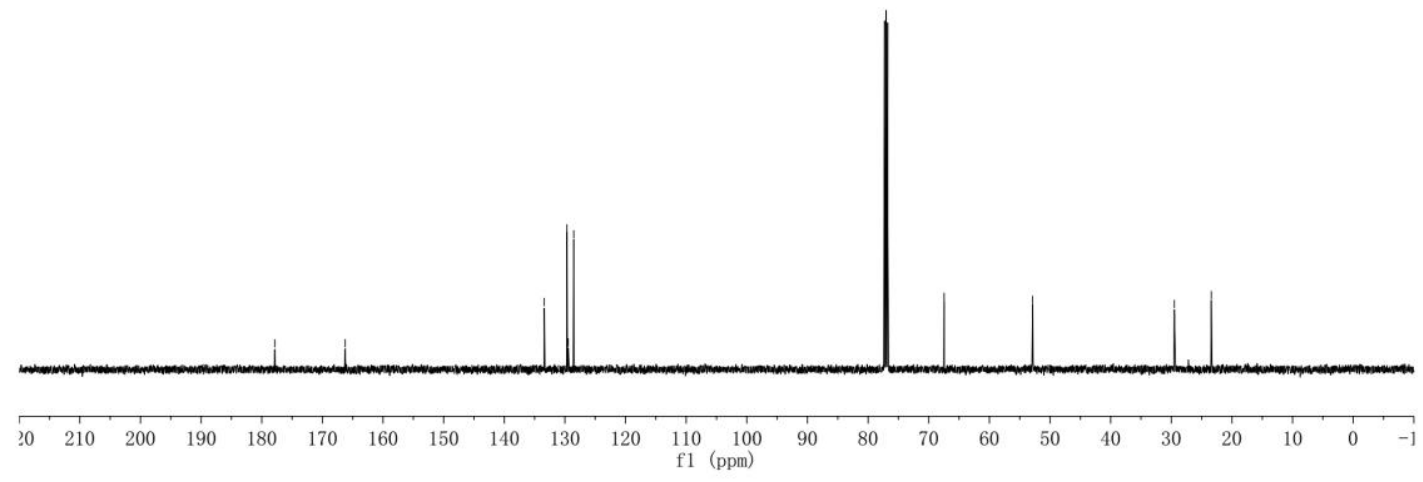



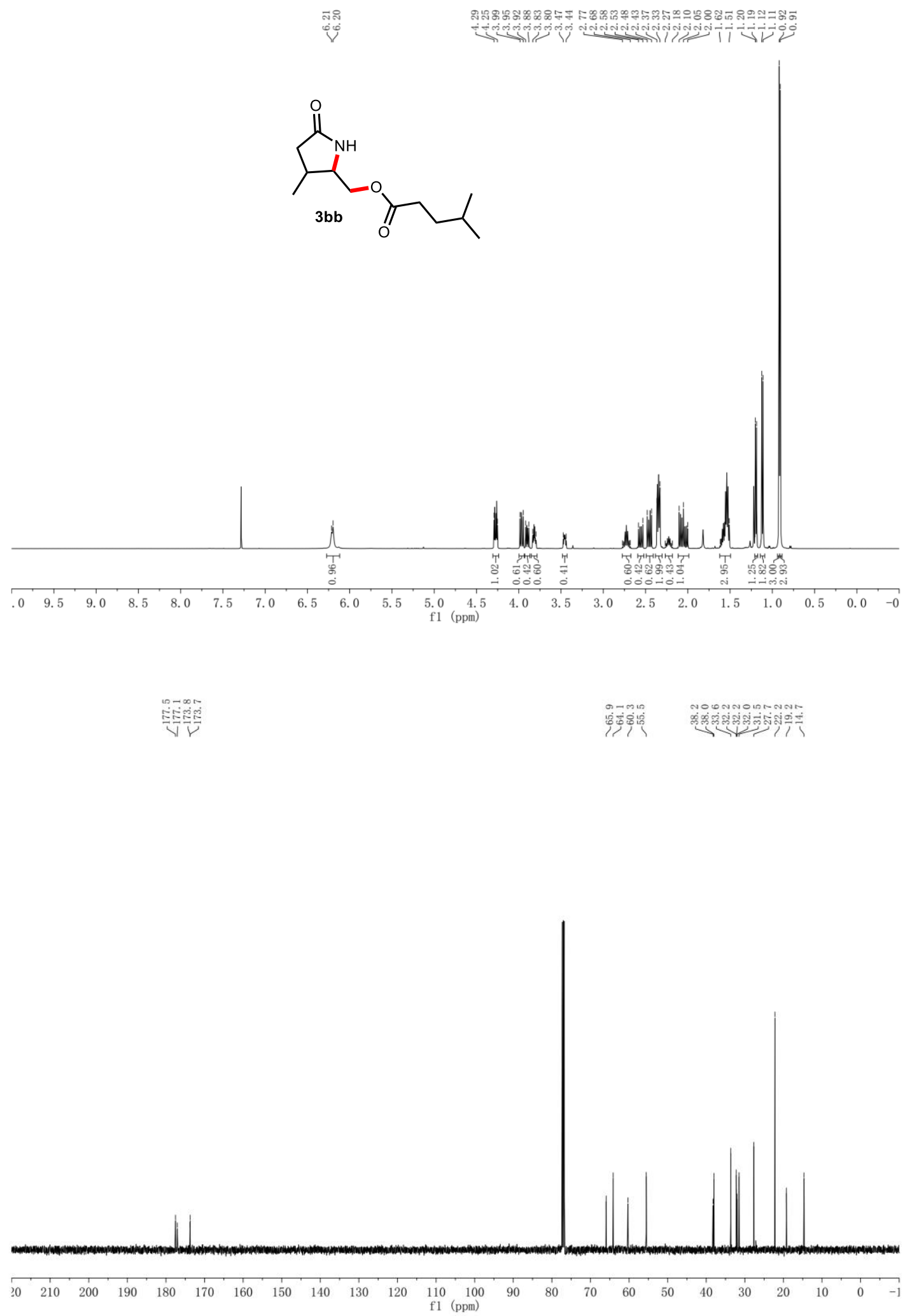

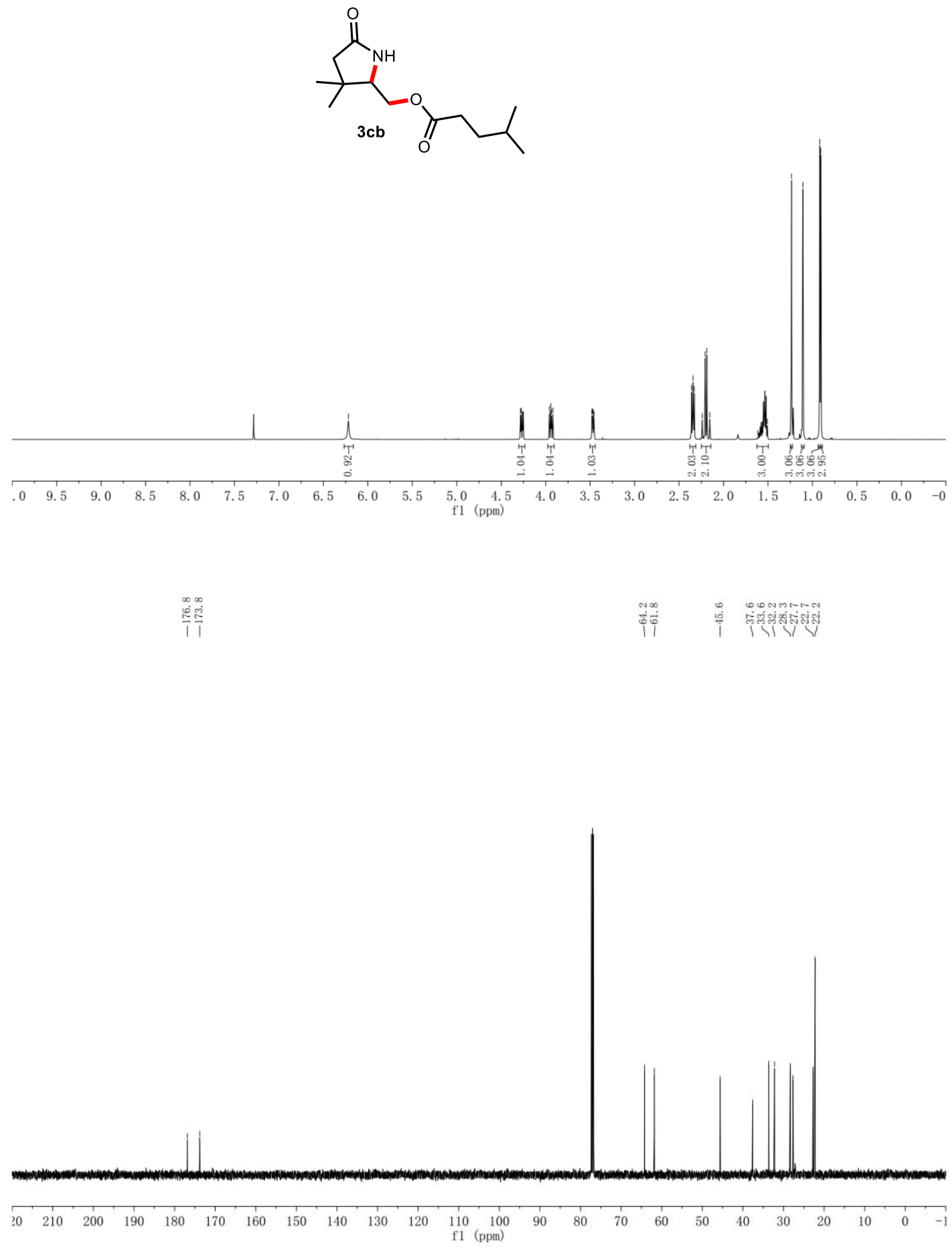

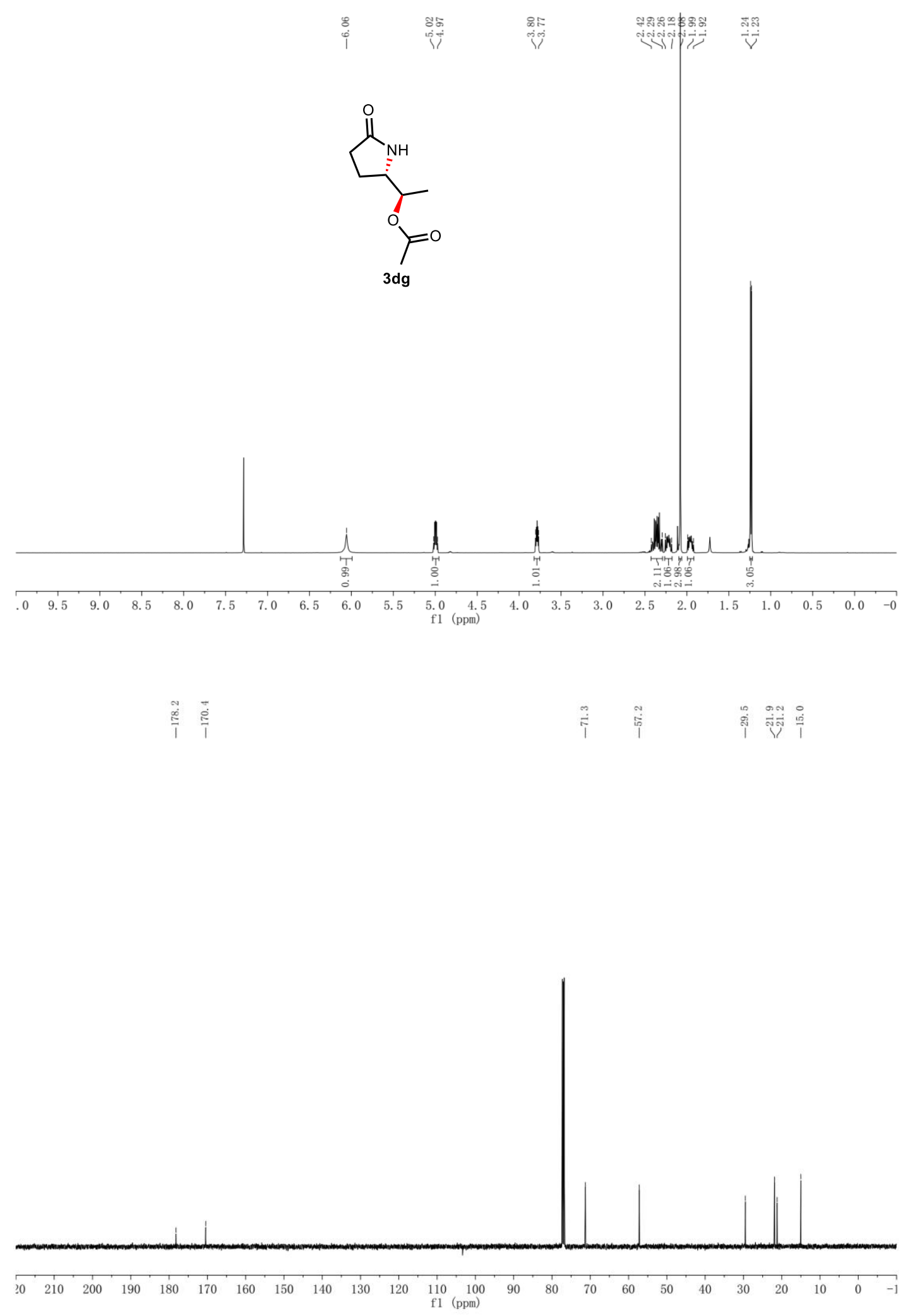


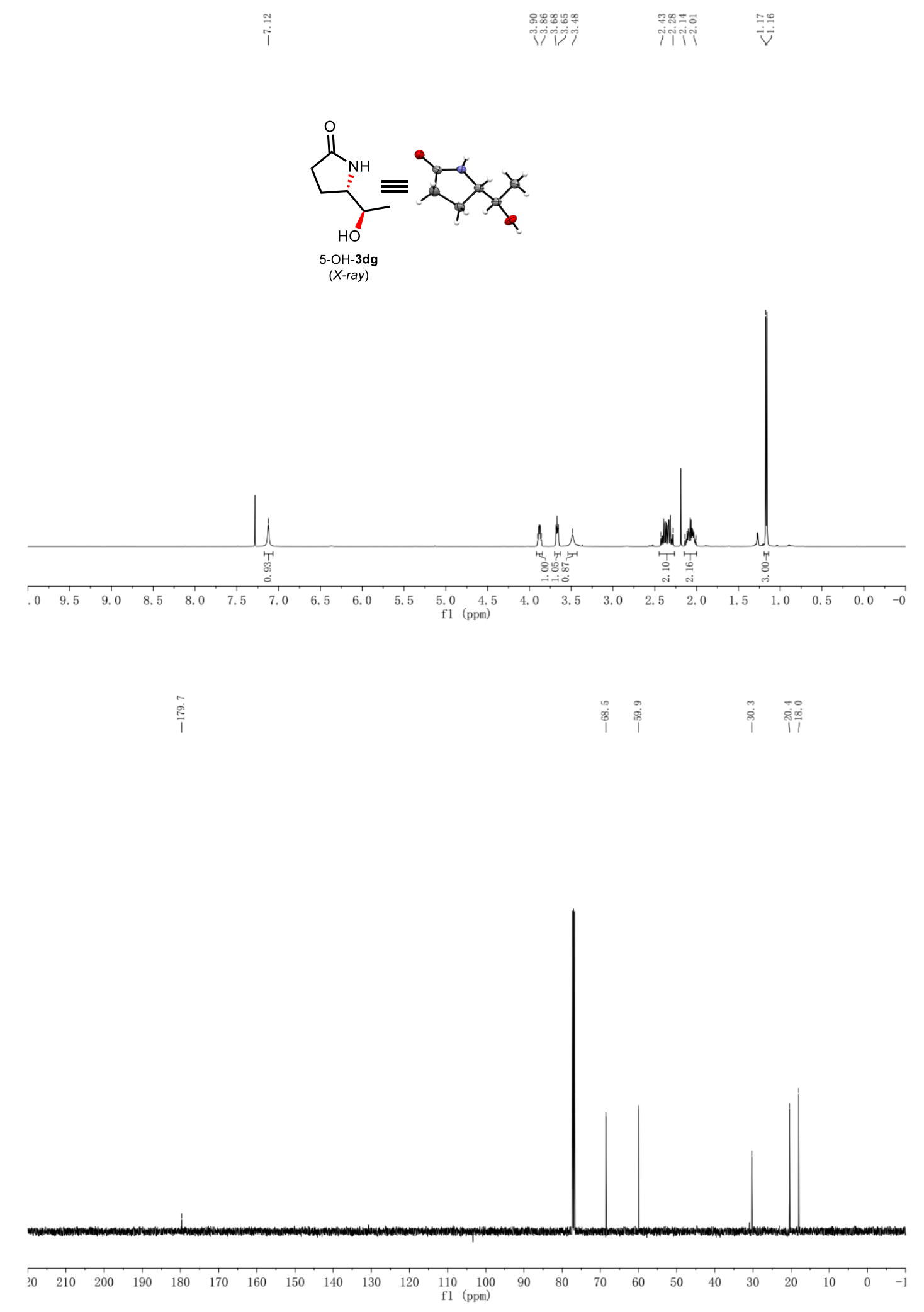



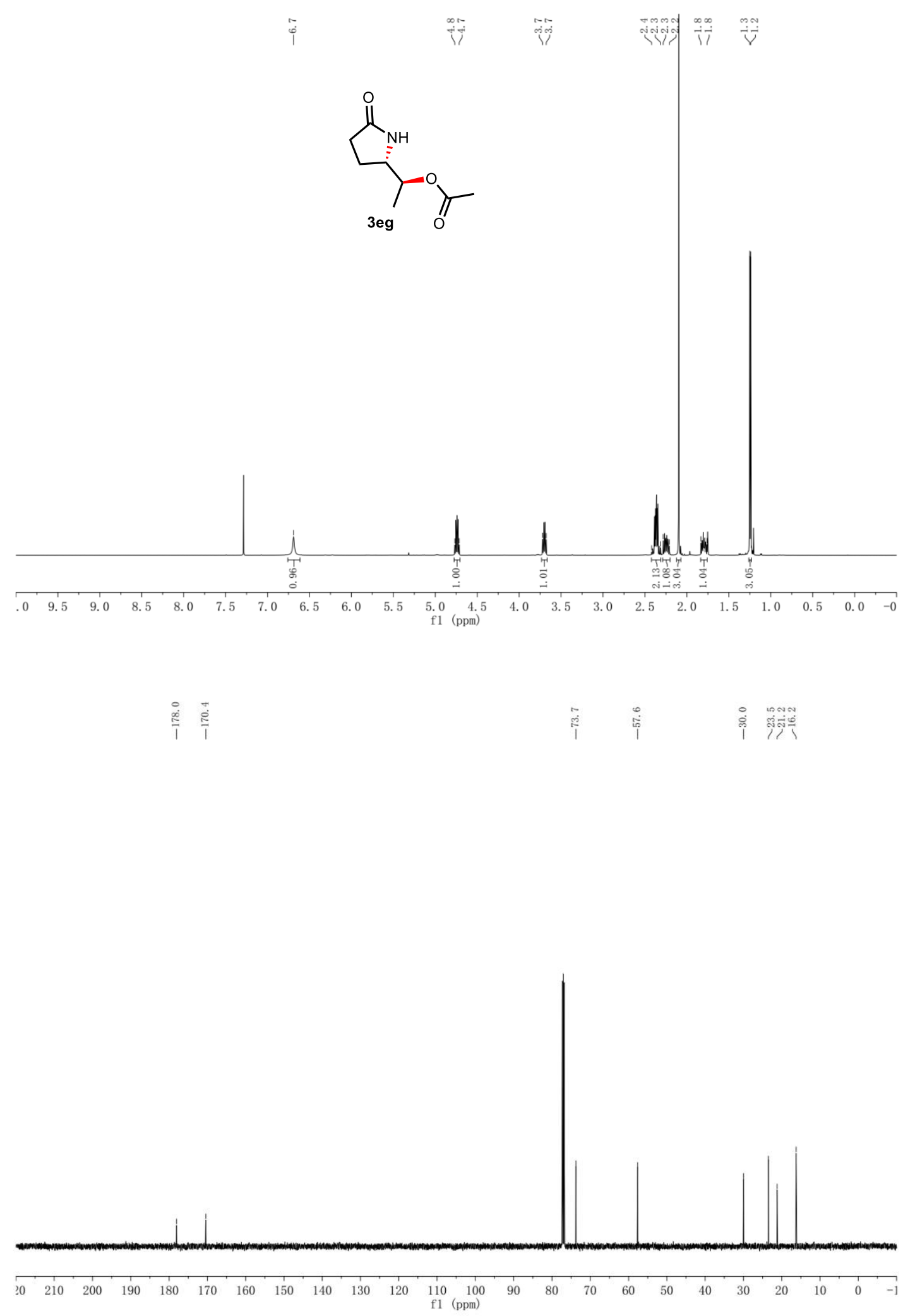

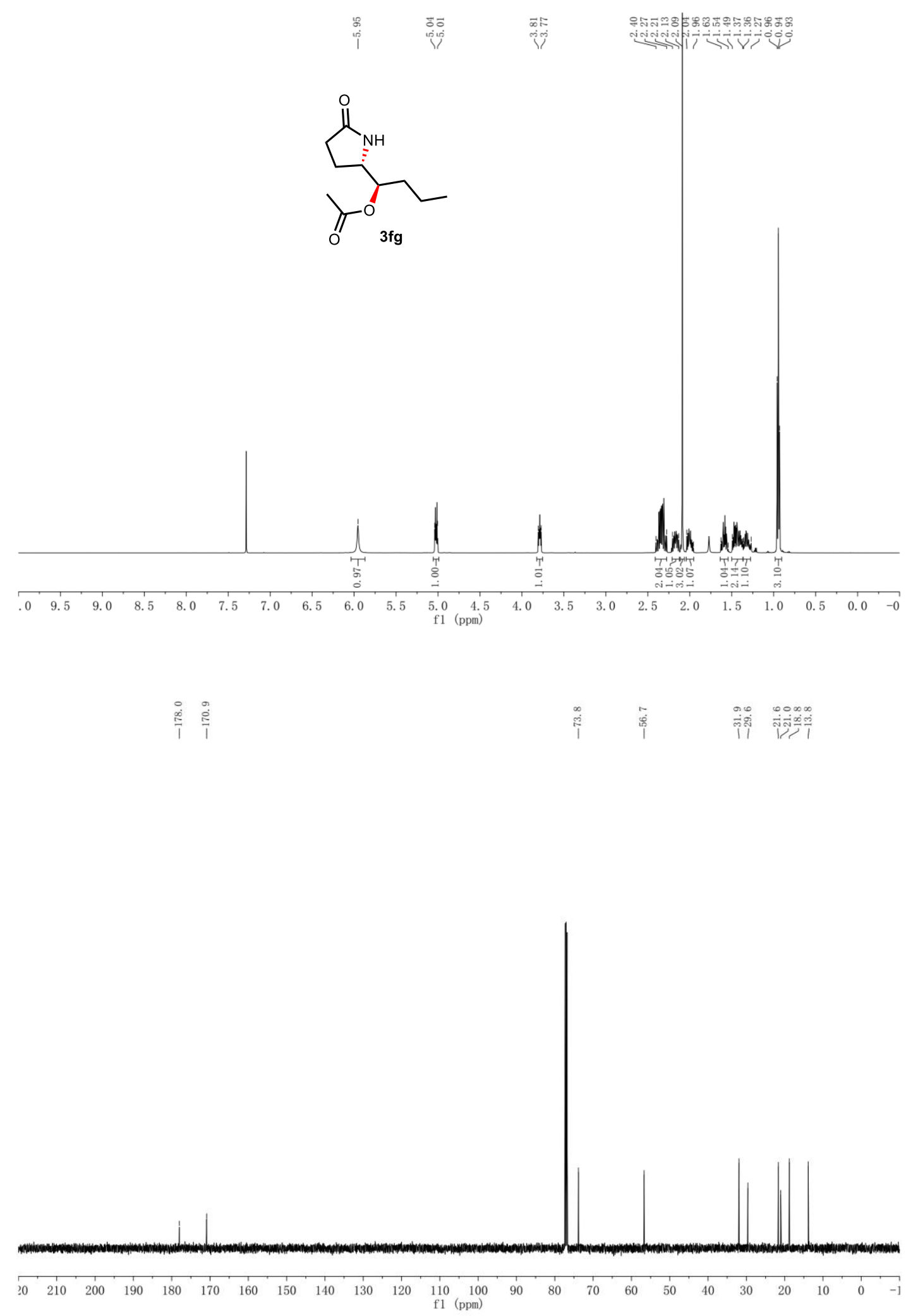

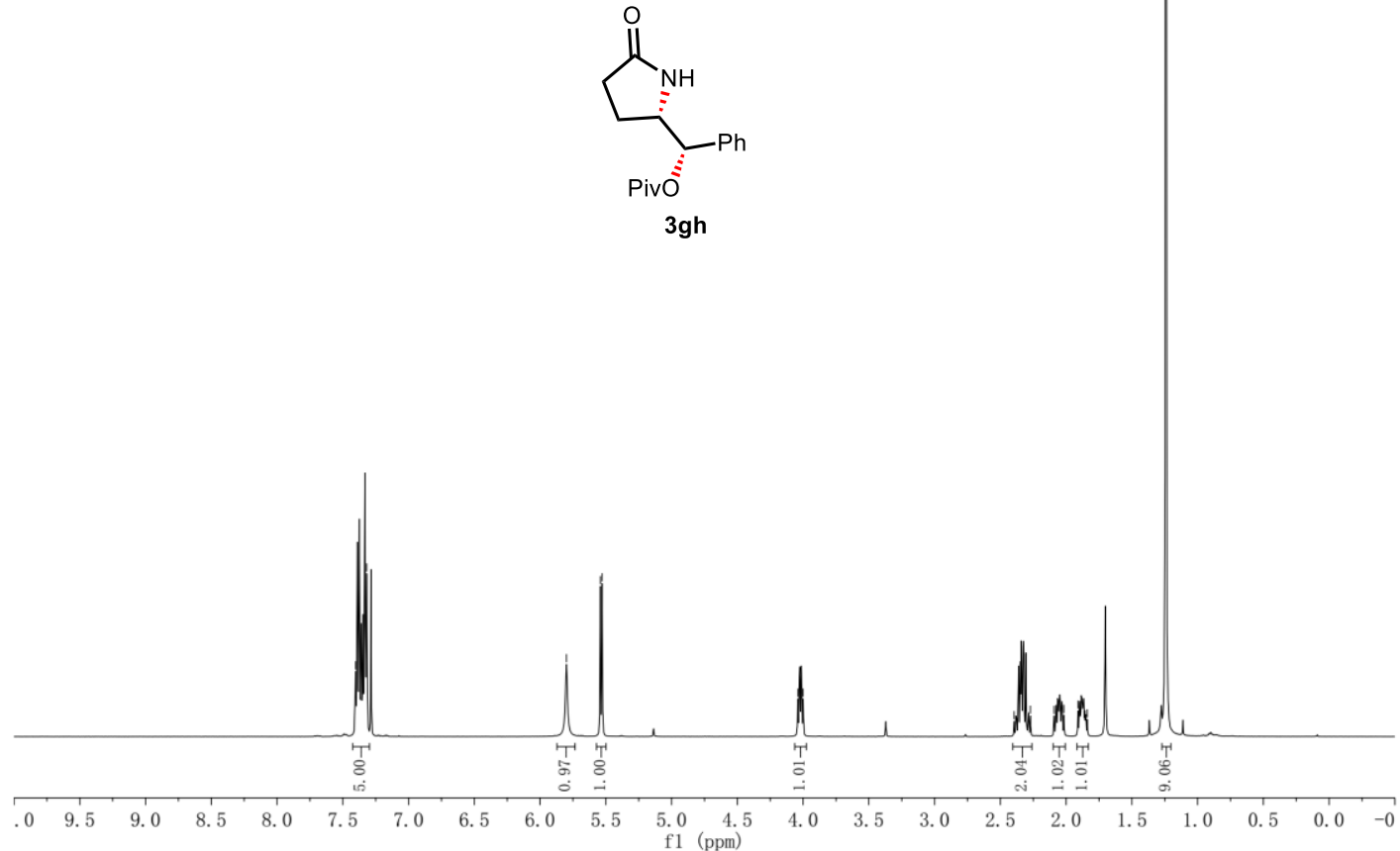

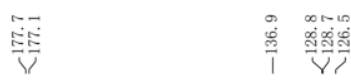

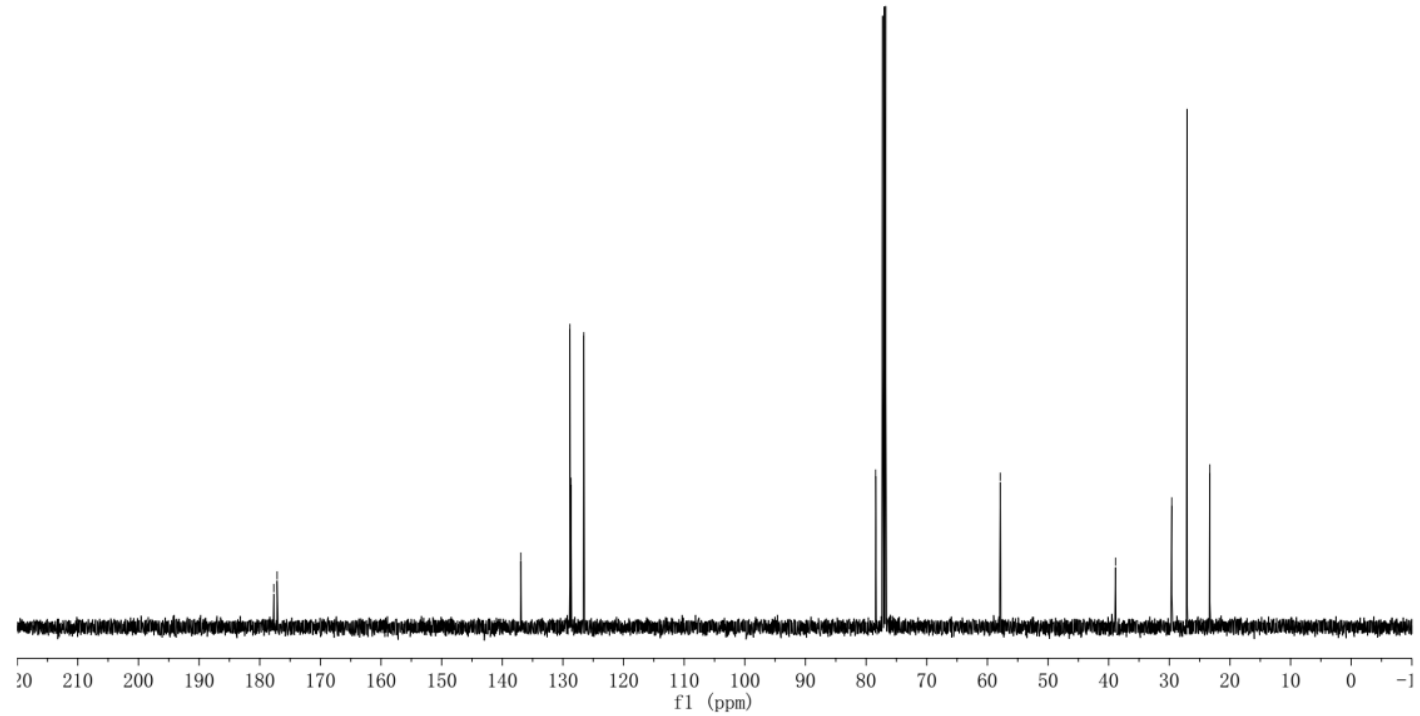




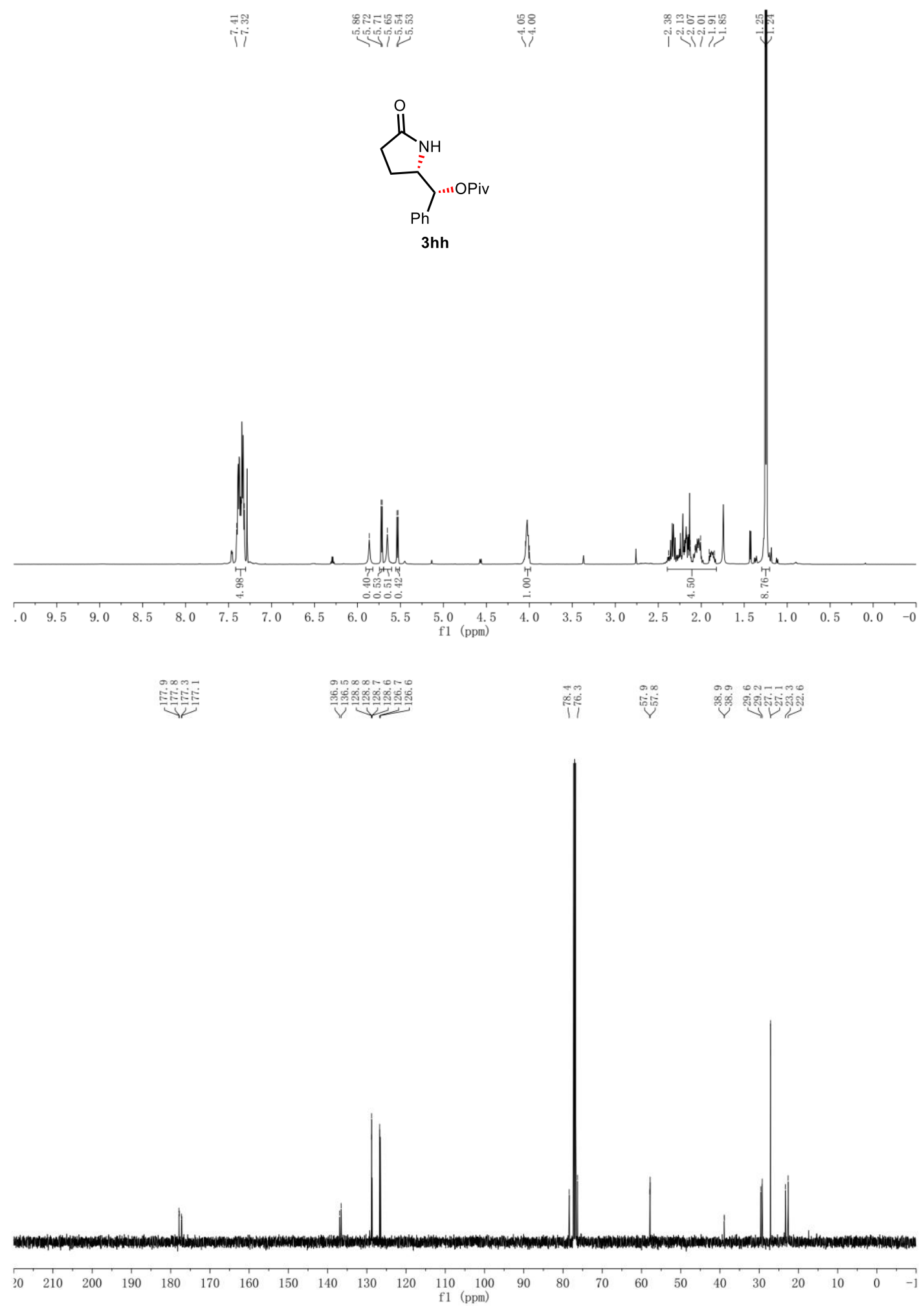



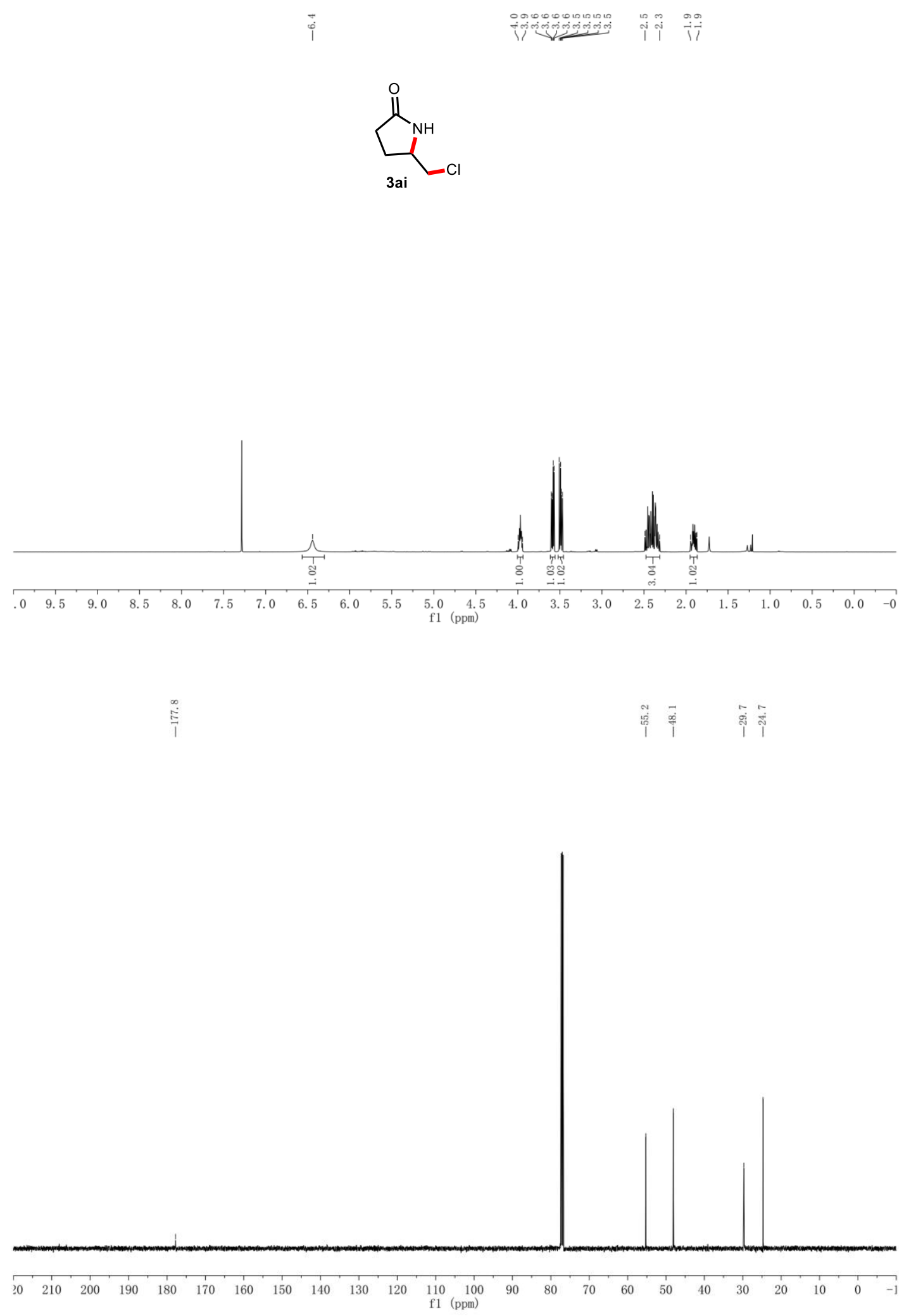

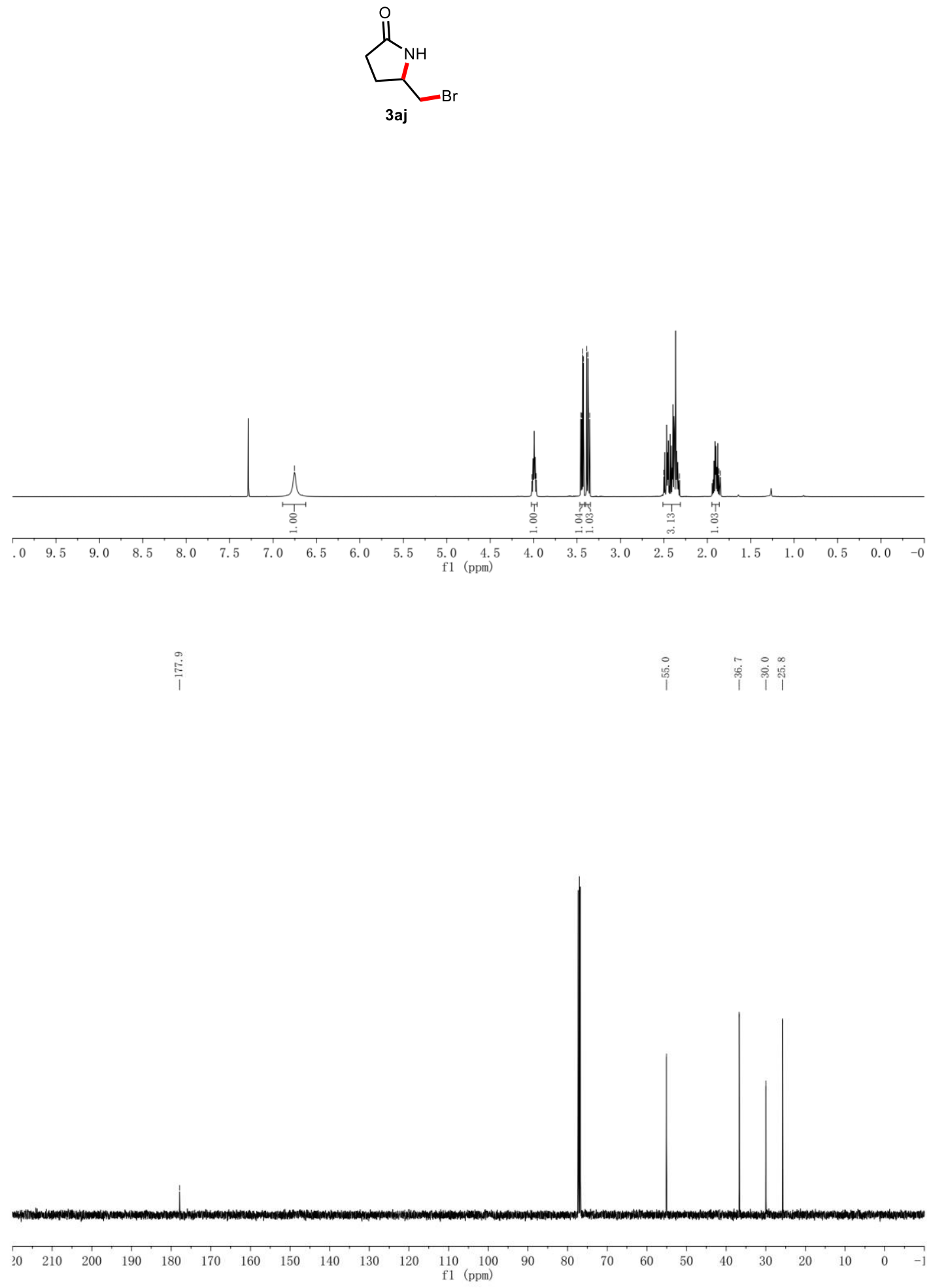


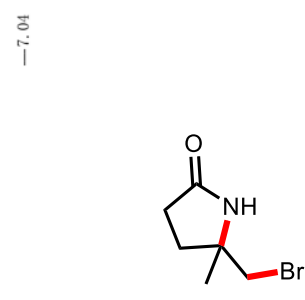

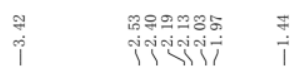

3ij
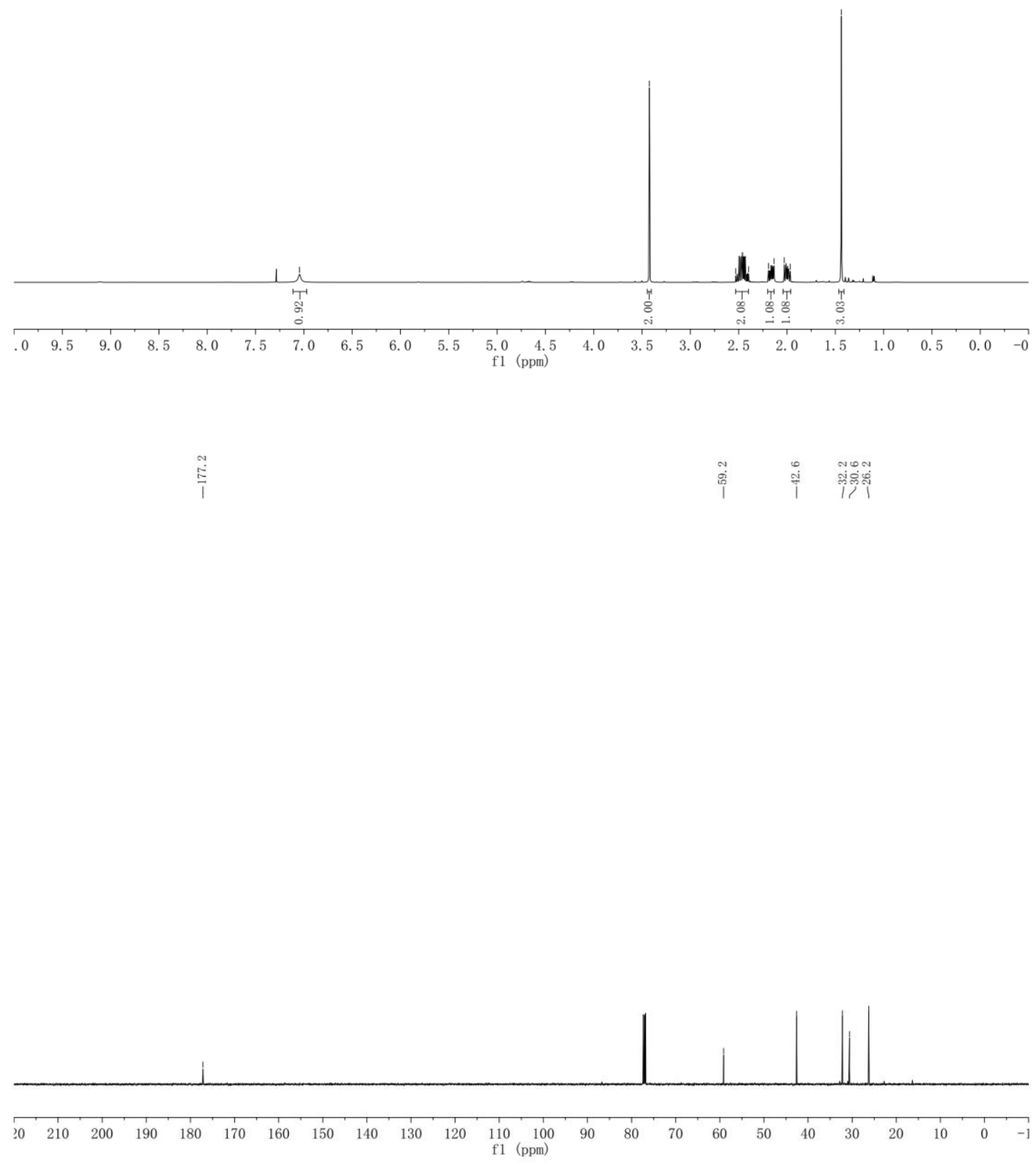


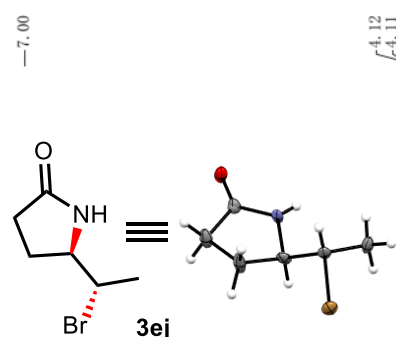

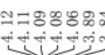

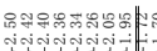

$\mathrm{Br} \quad 3 \mathrm{e}$

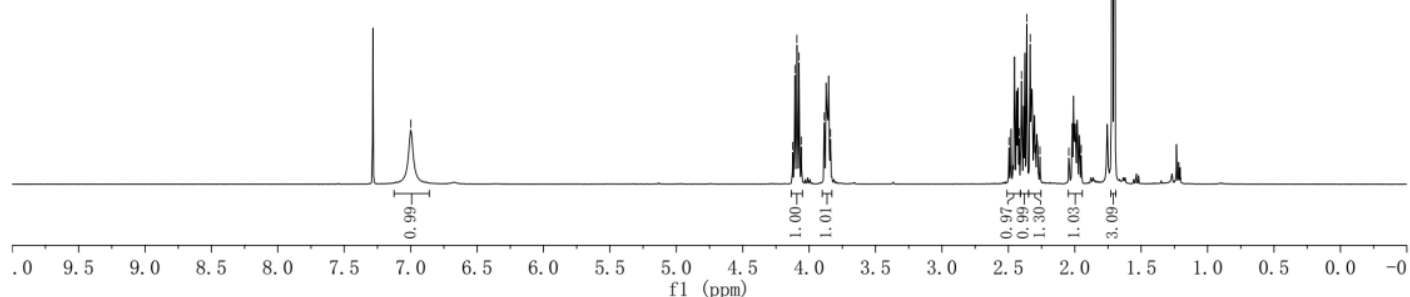

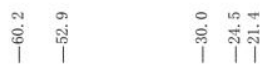

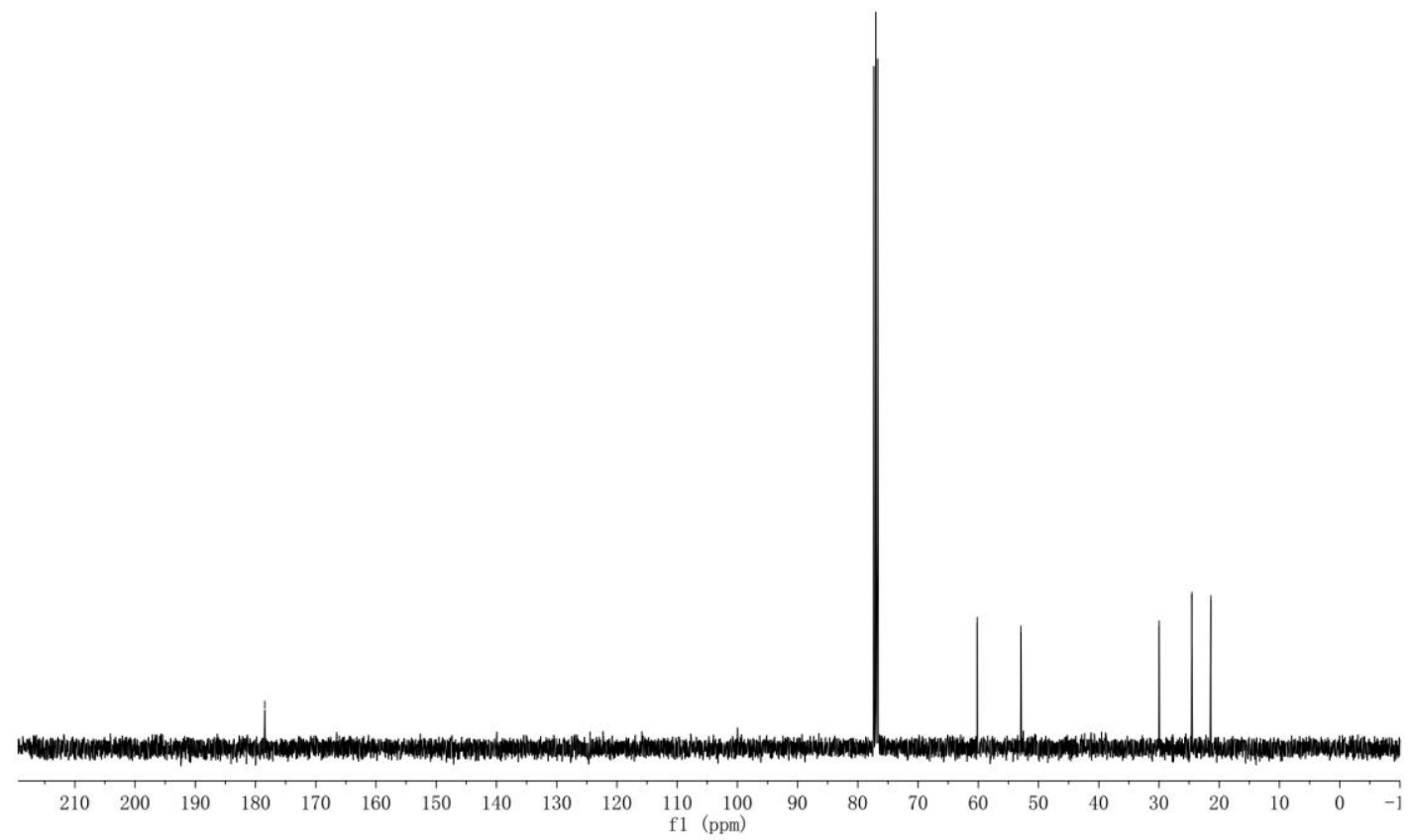




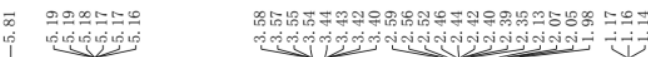<smiles>CCC(=O)OC1CCC(=O)NC1</smiles>

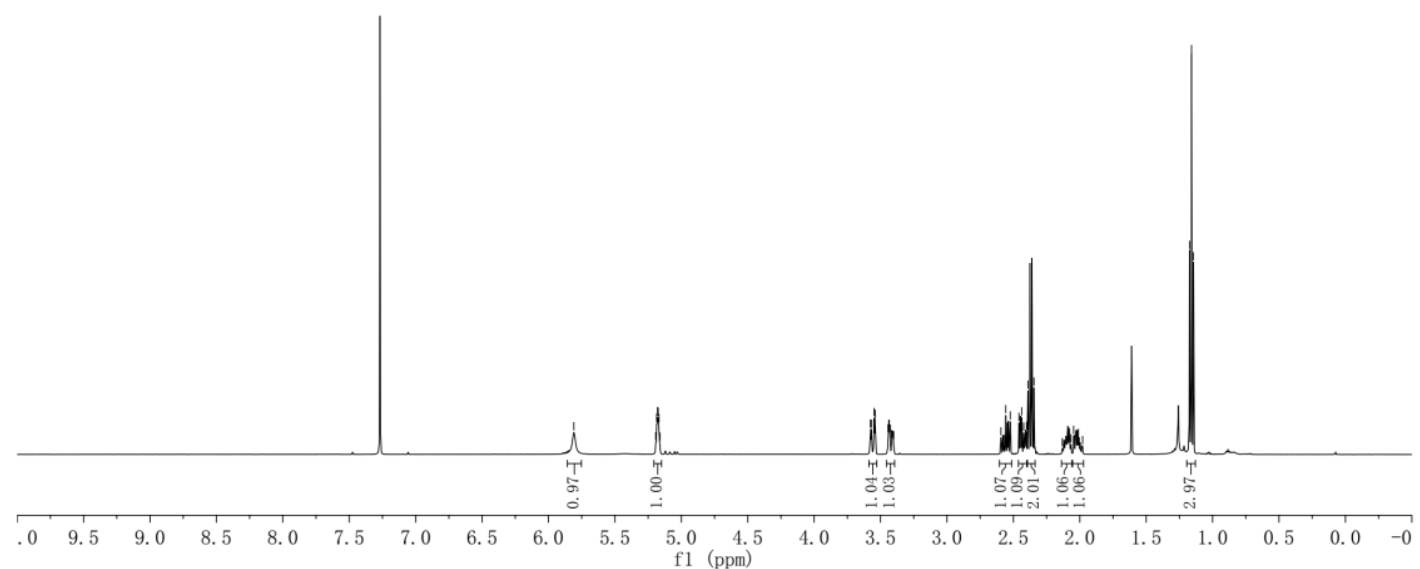


<smiles>CC(C)CCC(=O)OC1CCC(=O)NC1</smiles>
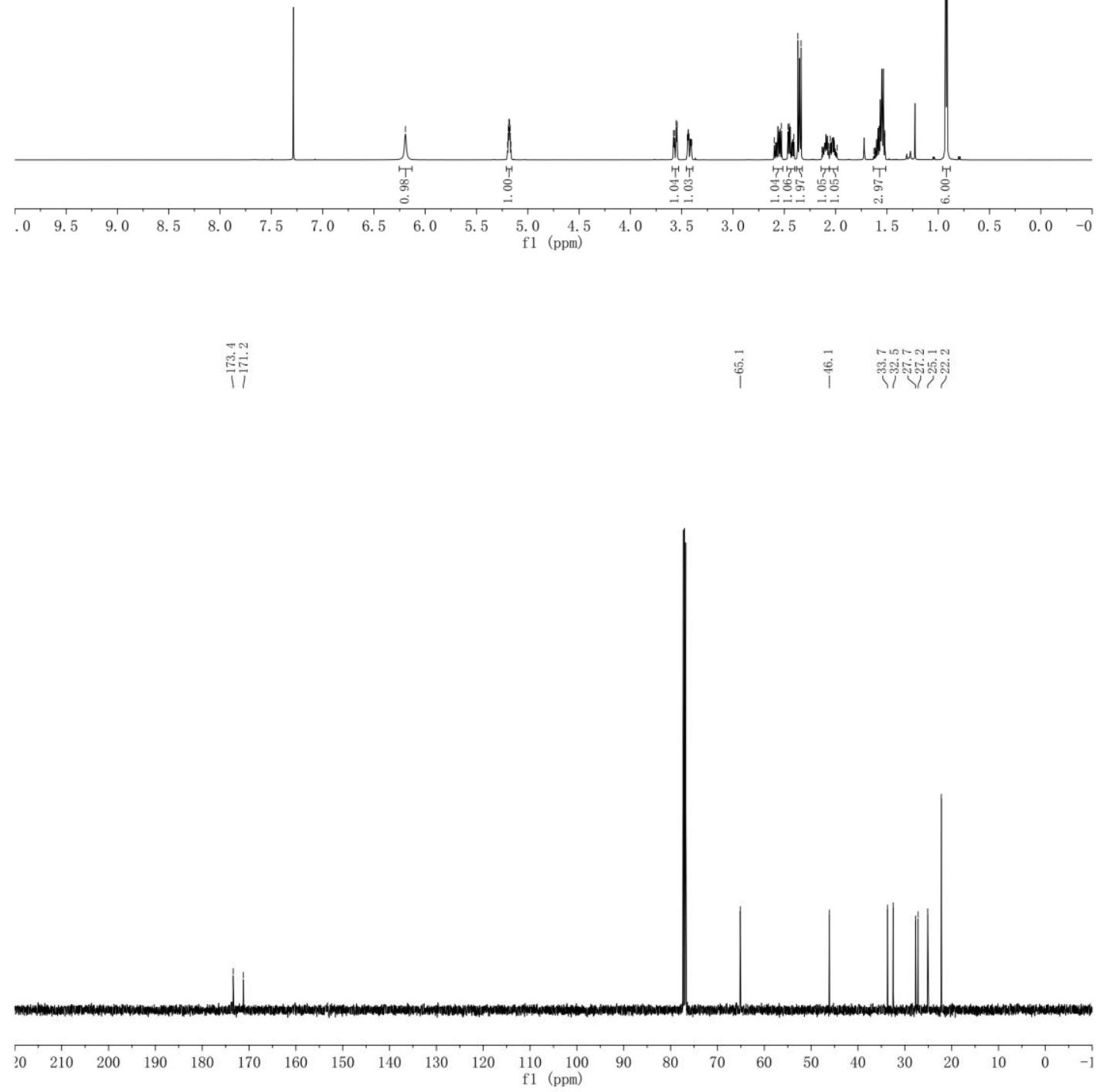


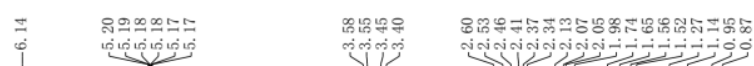<smiles>O=C1CCC(OC(=O)CCC2CCCCC2)CN1</smiles>
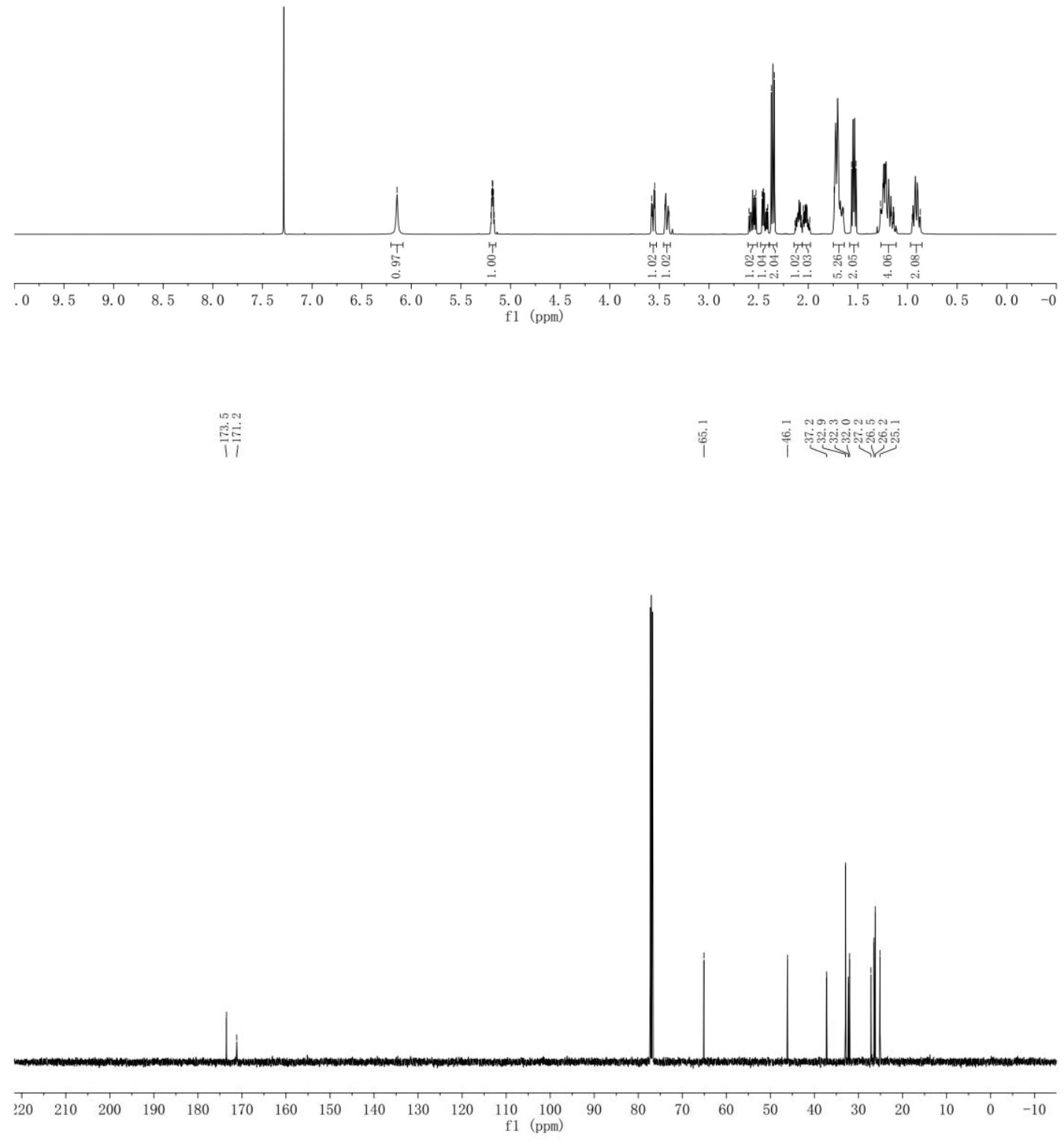


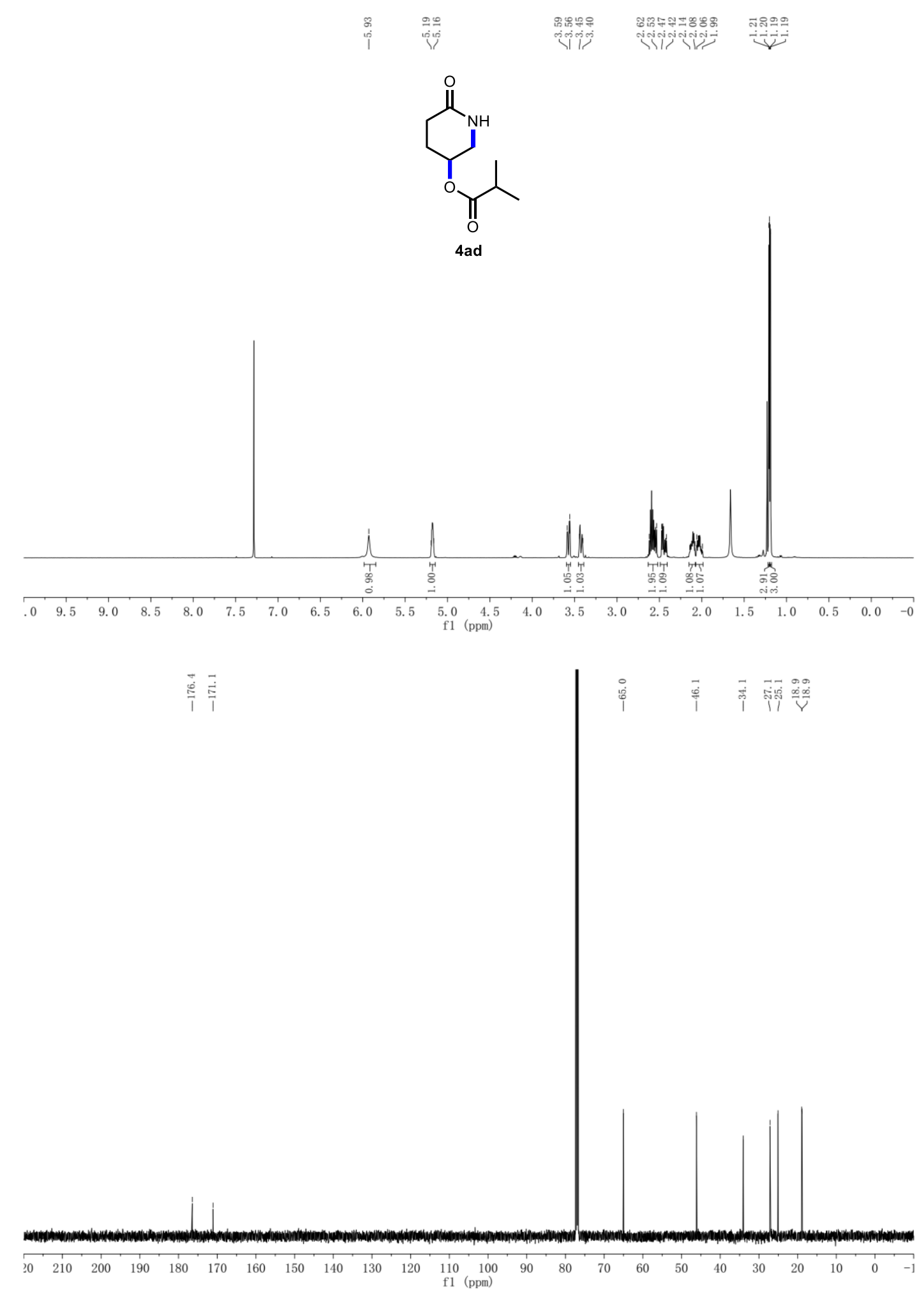



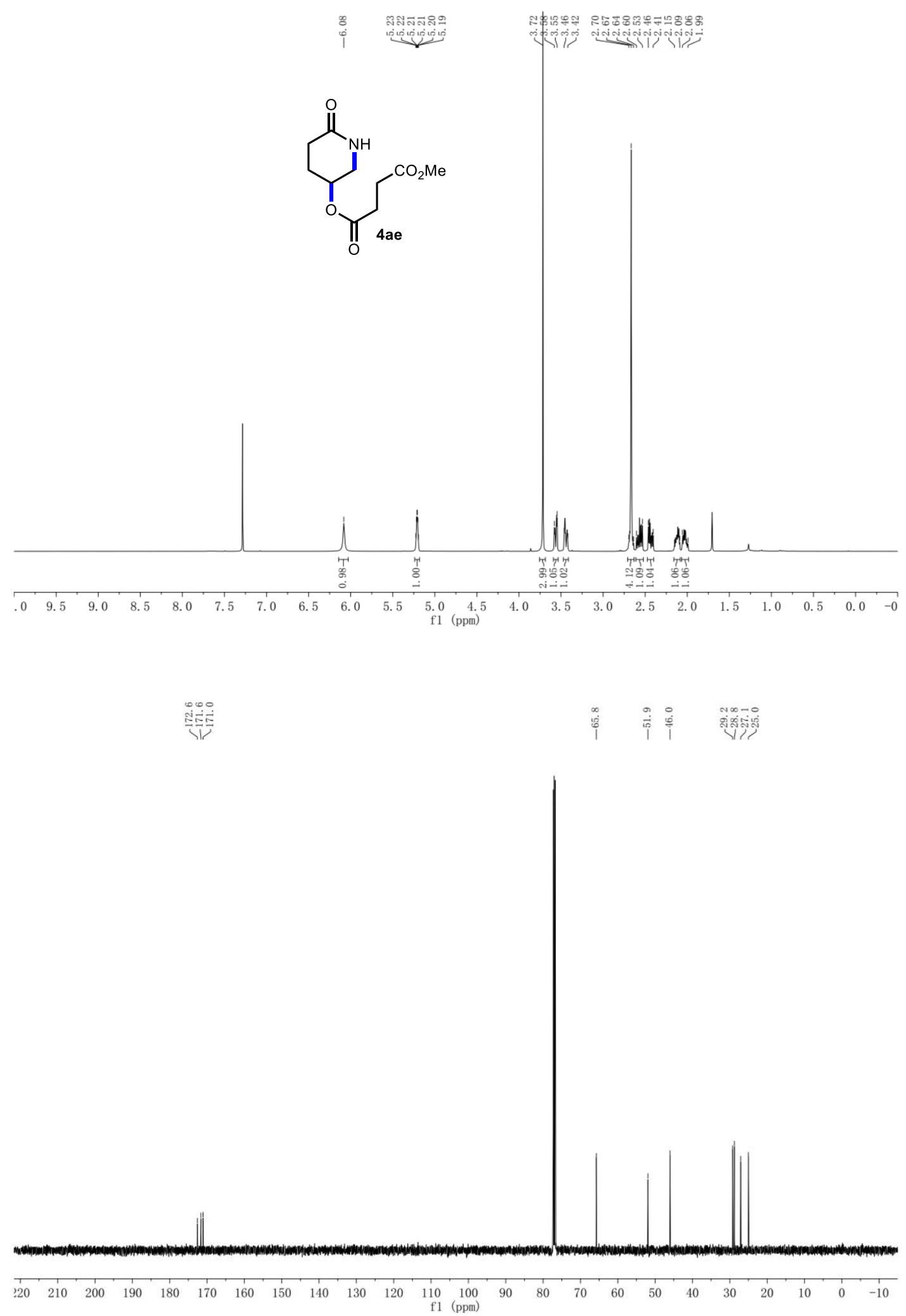

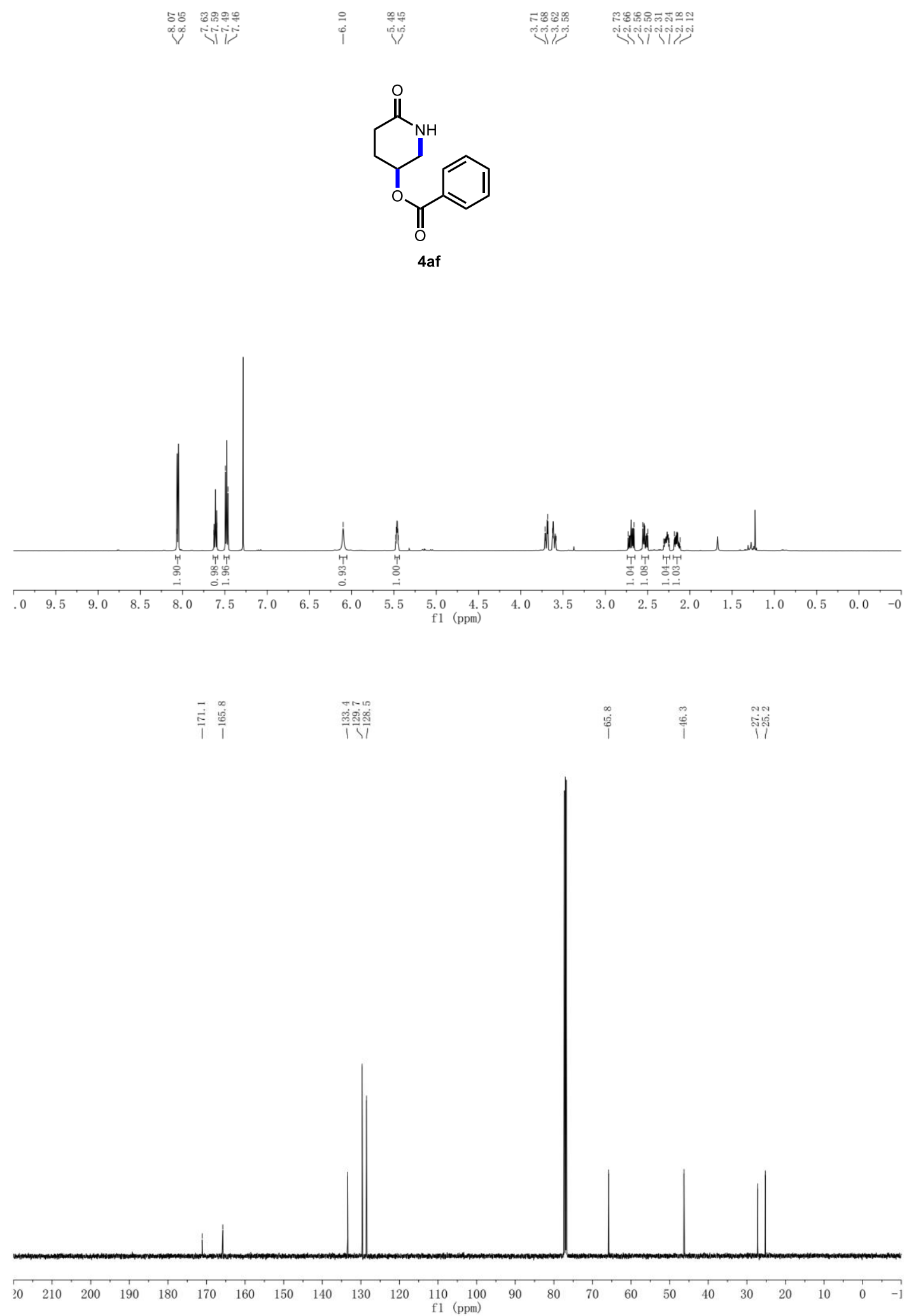

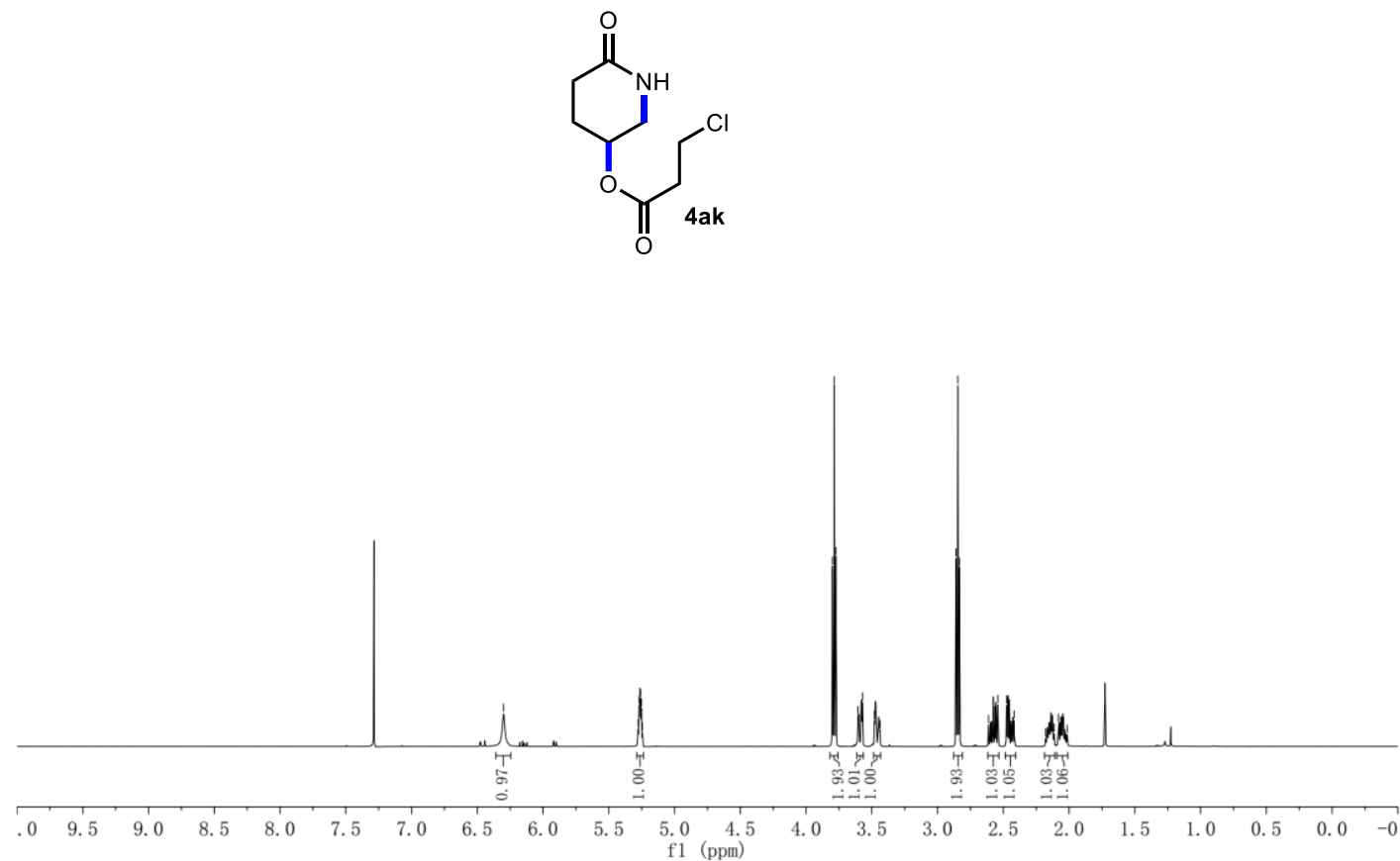

$\overrightarrow{\frac{1}{i}}$

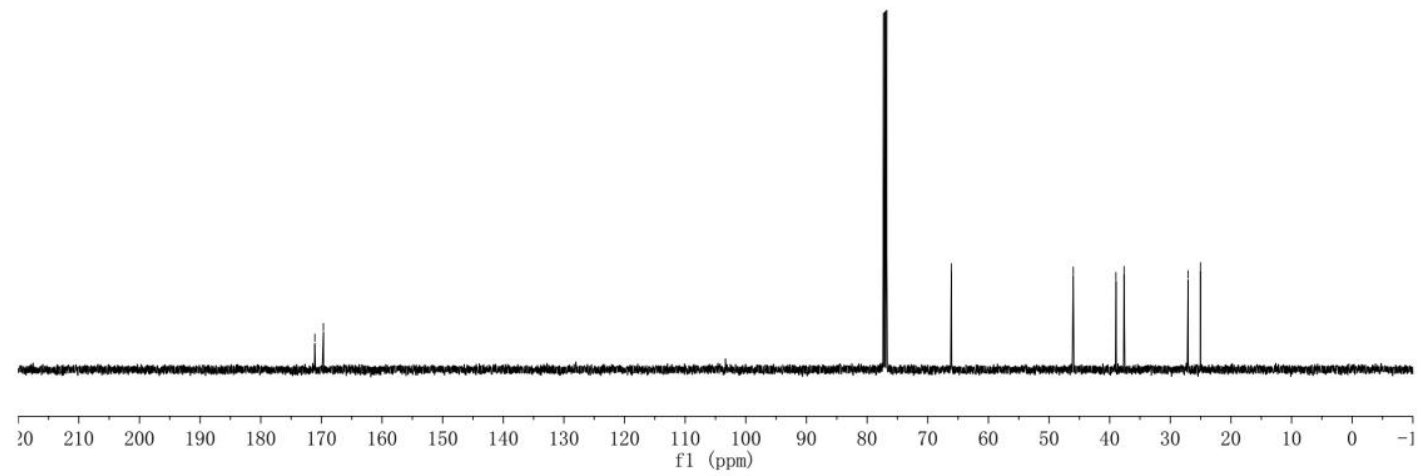



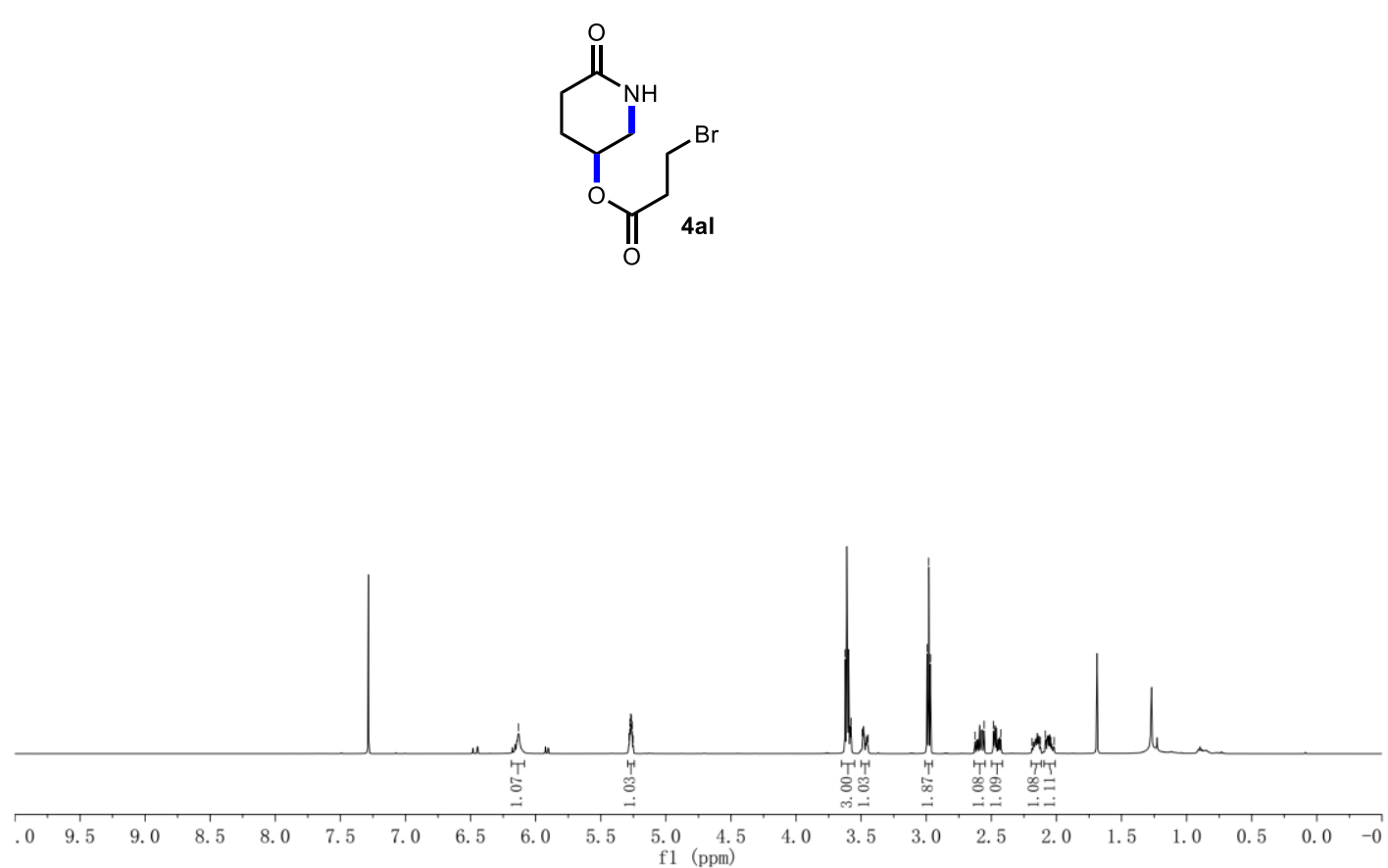

웅

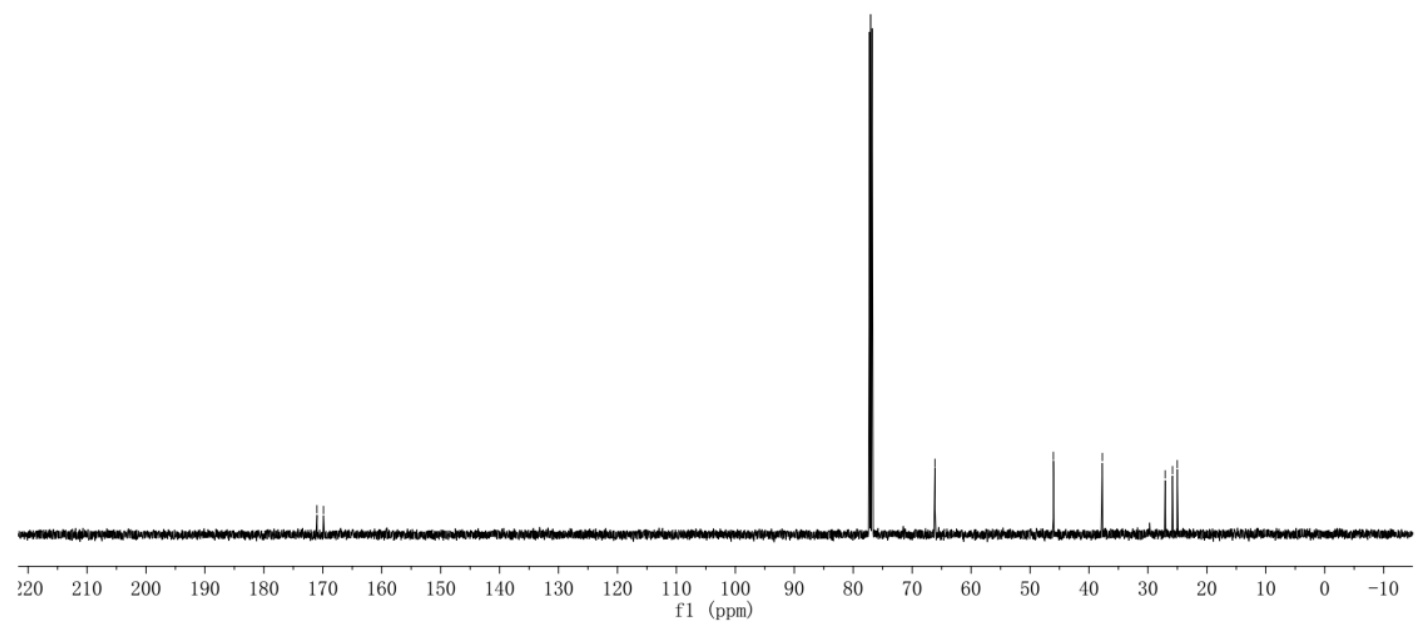



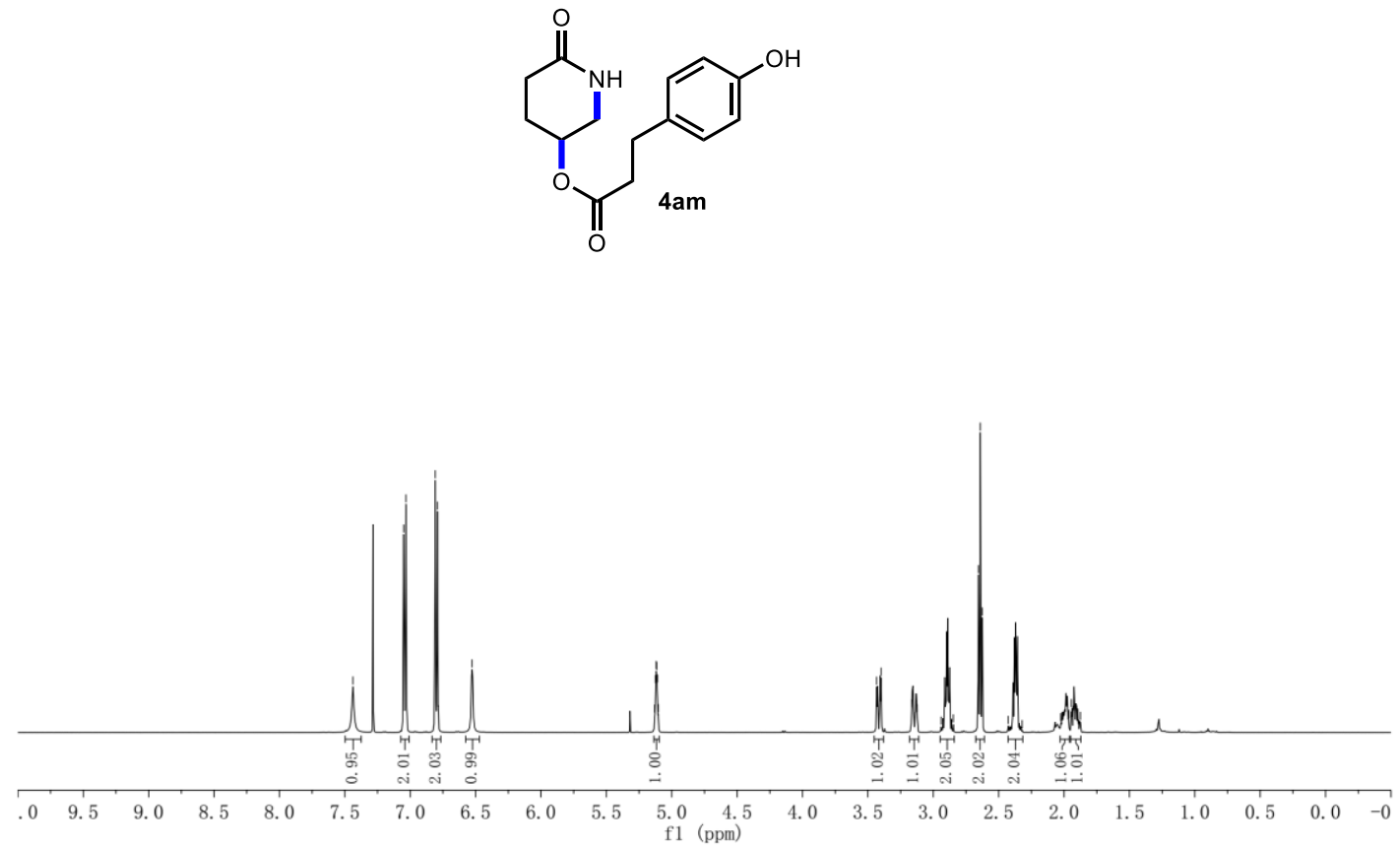

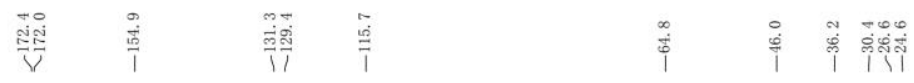

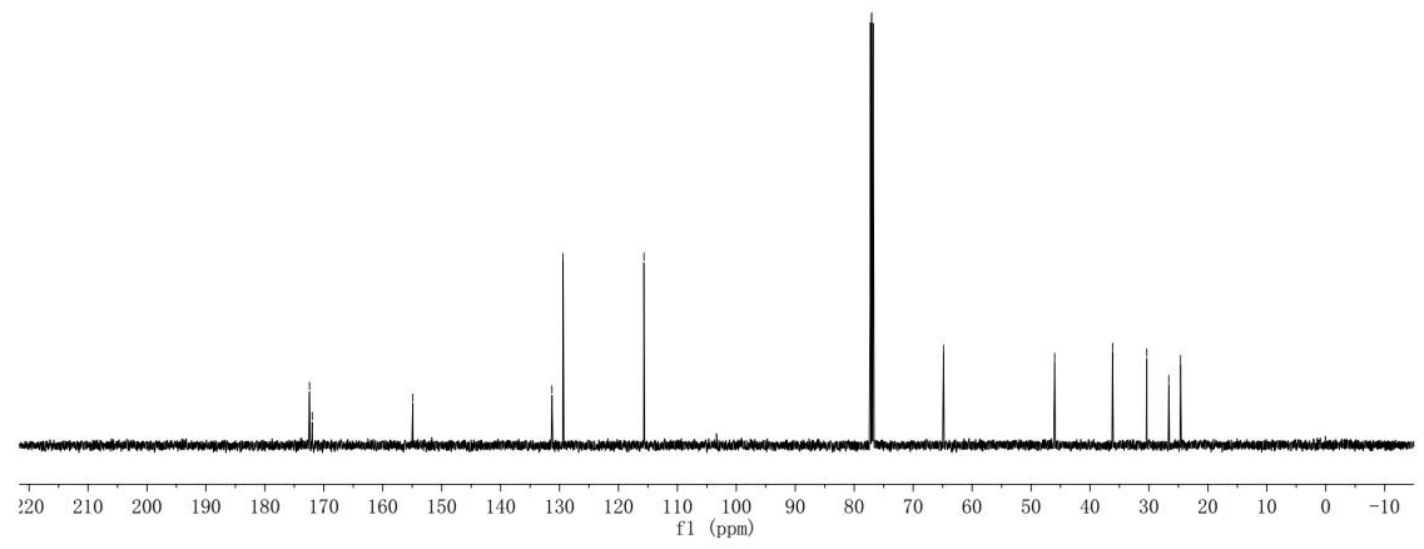




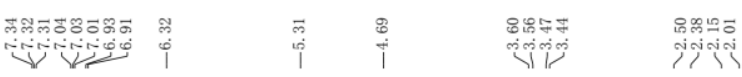<smiles>O=C1CCC(OC(=O)COc2ccccc2)CN1</smiles>

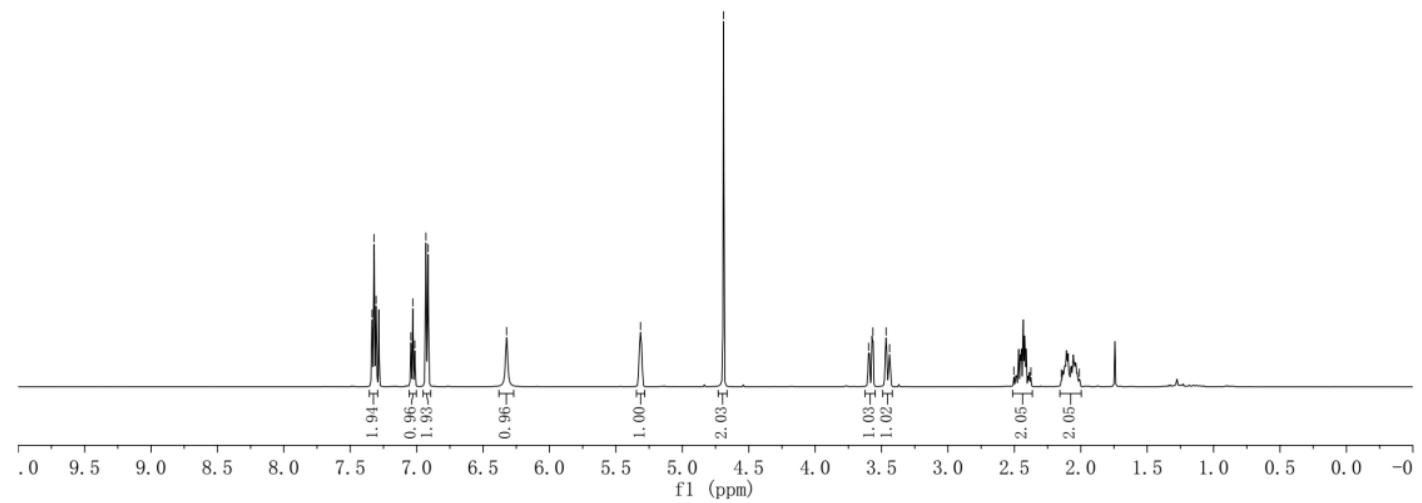

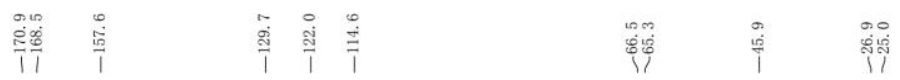

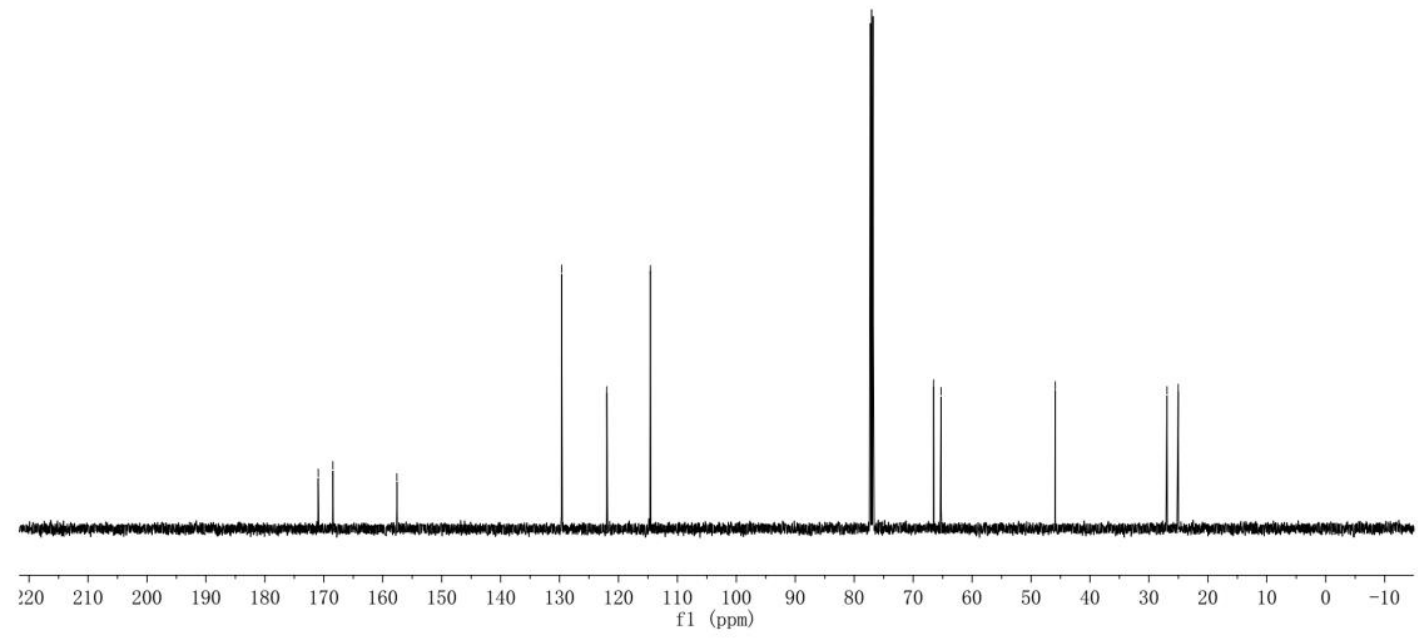




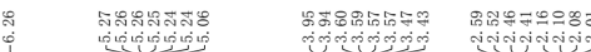
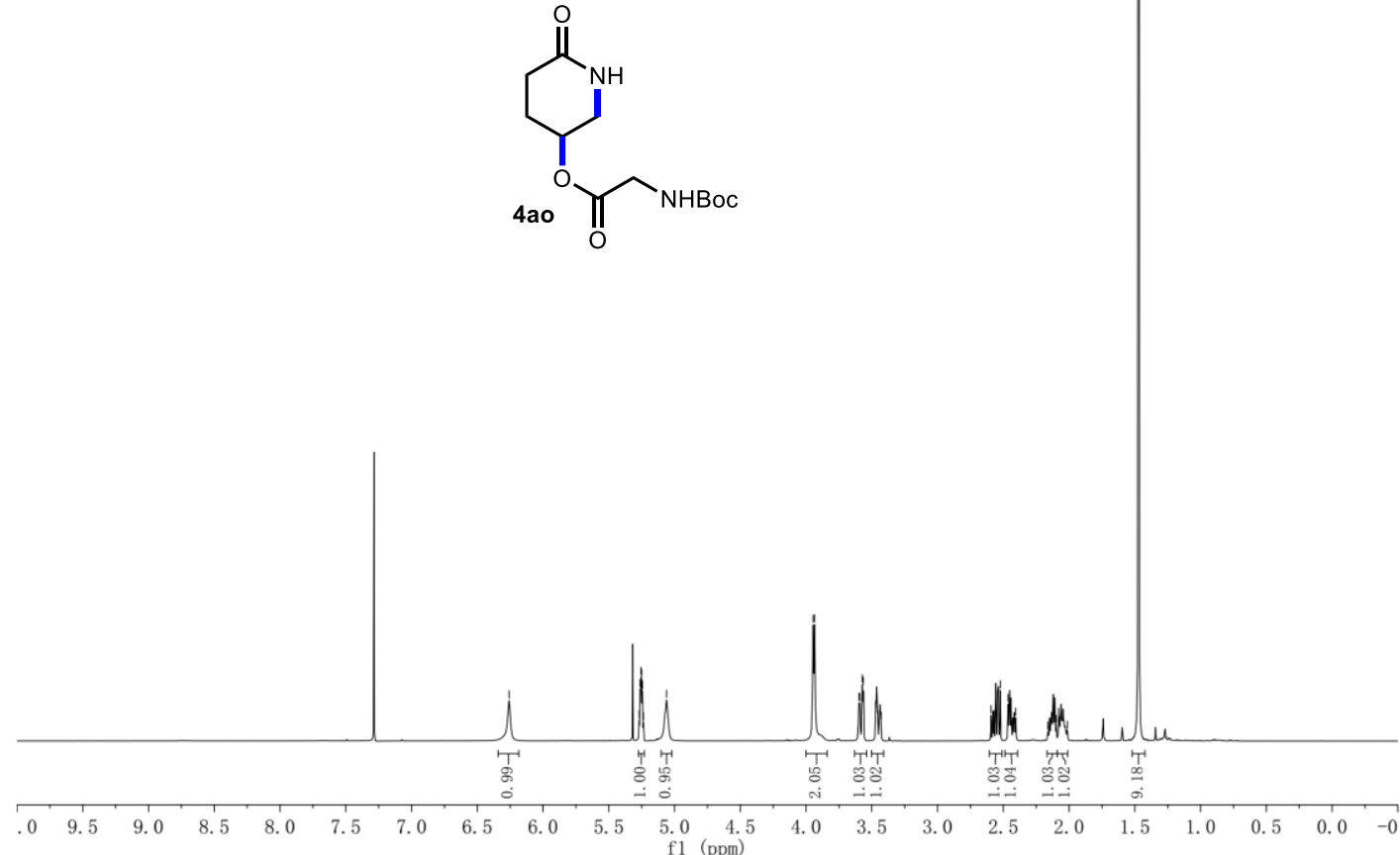

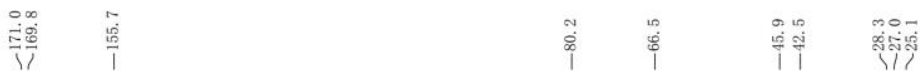

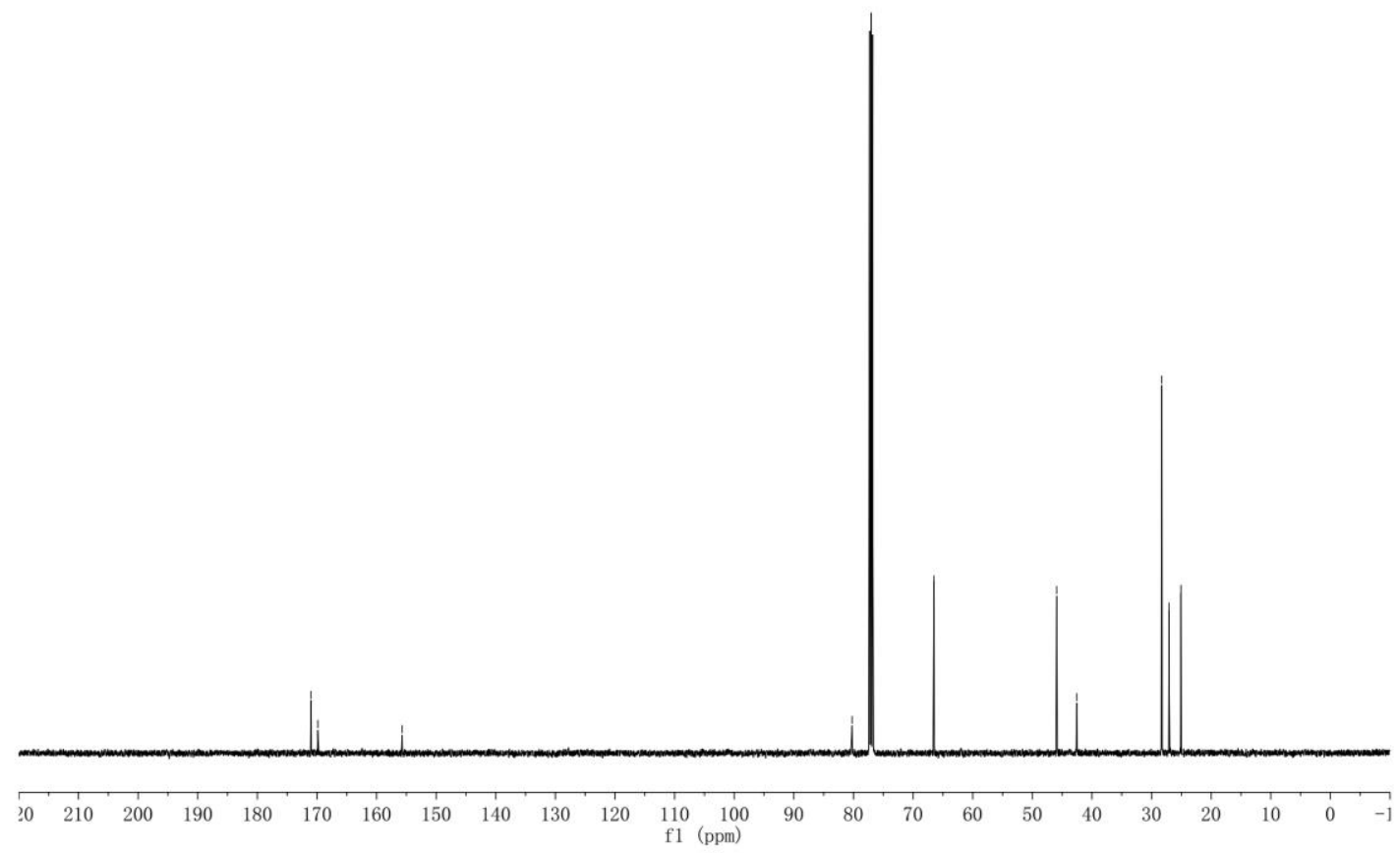




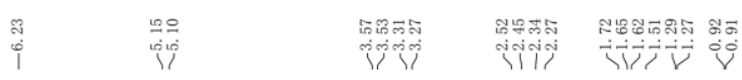<smiles>CC(C)CCC(=O)O[C@@H]1CNC(=O)[C@H](C)C1</smiles>

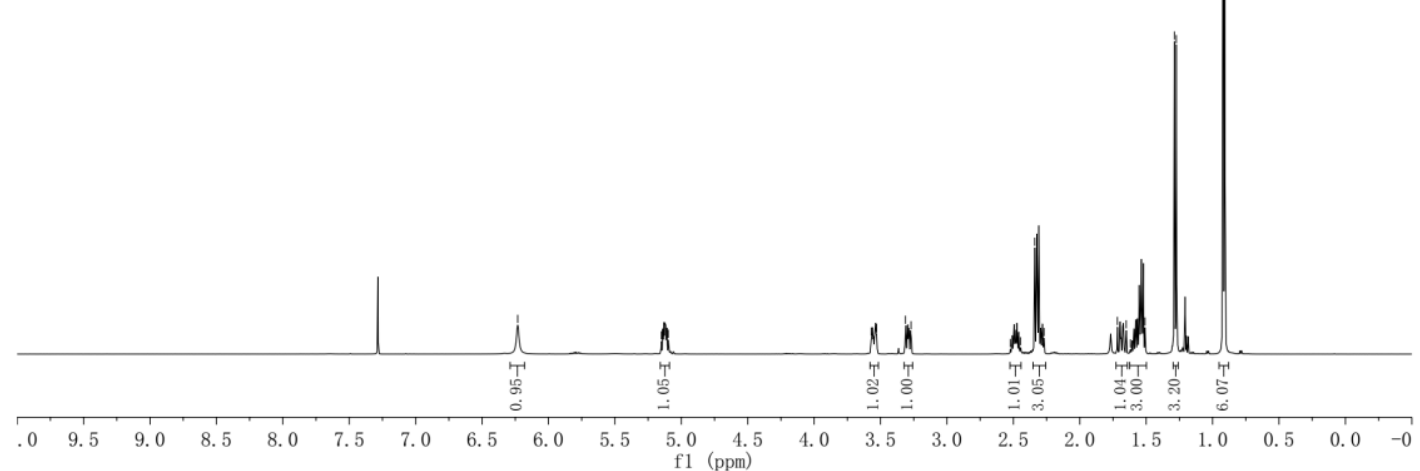

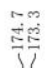

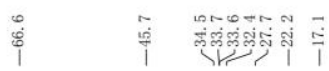

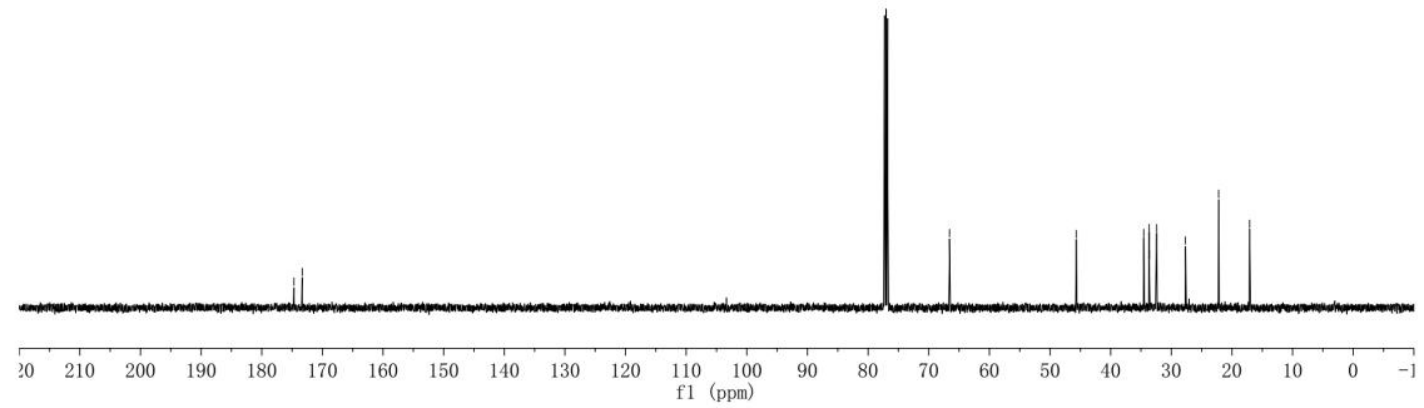



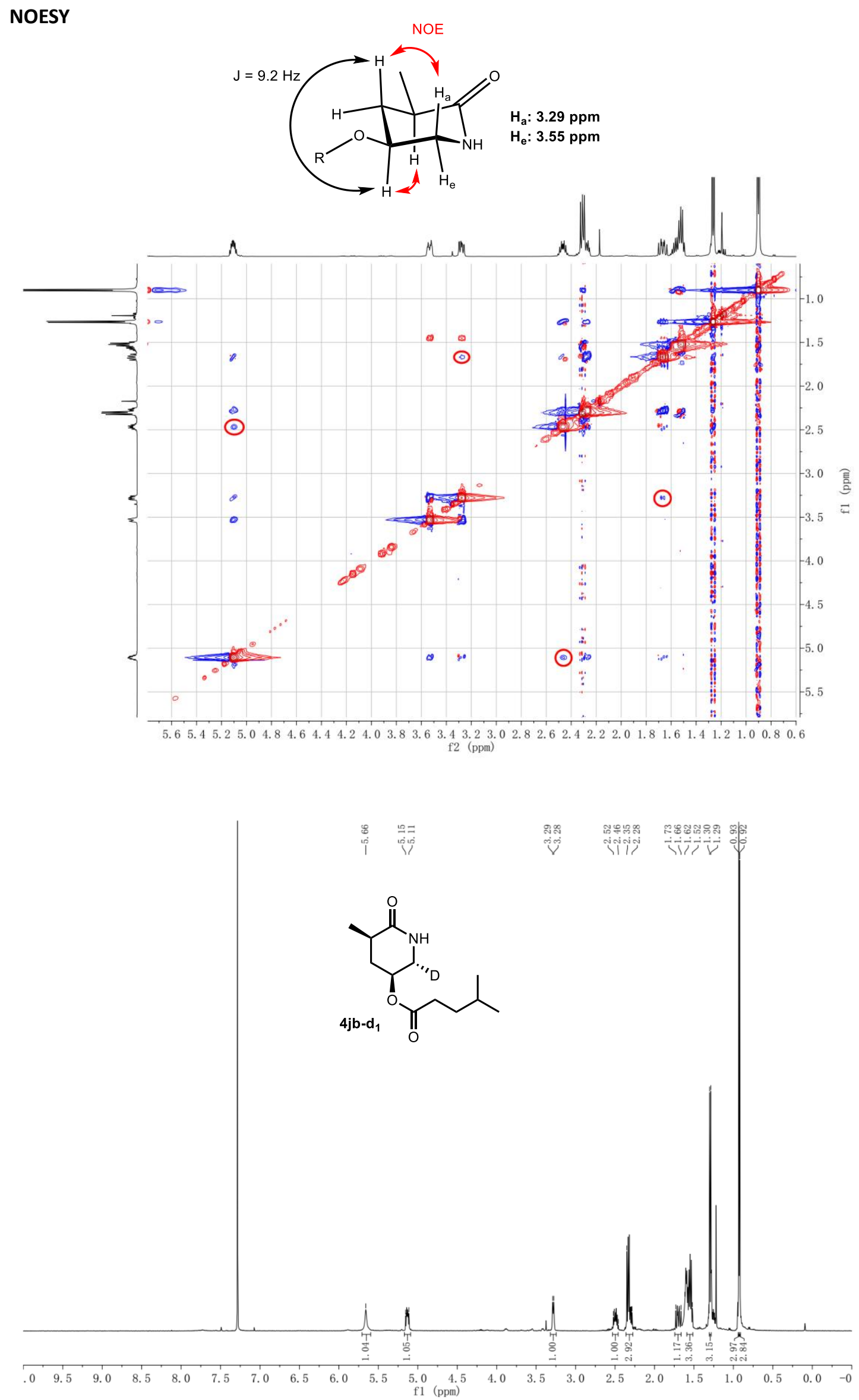
<smiles>CC(C)CCC(=O)OC1CNC(=O)C(Cc2ccc(Oc3ccccc3)cc2)C1</smiles>

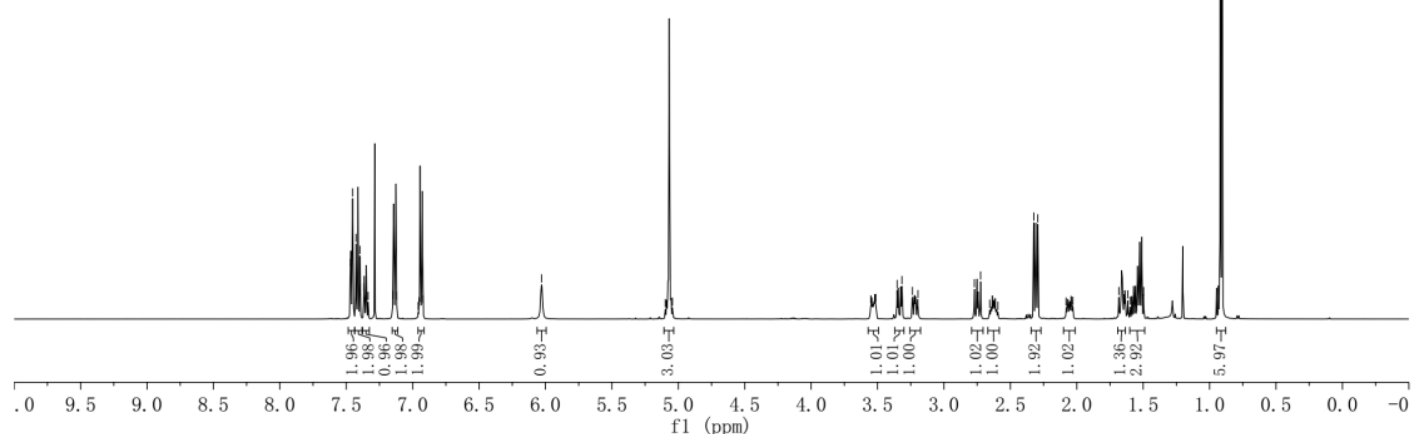

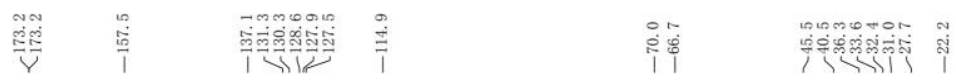

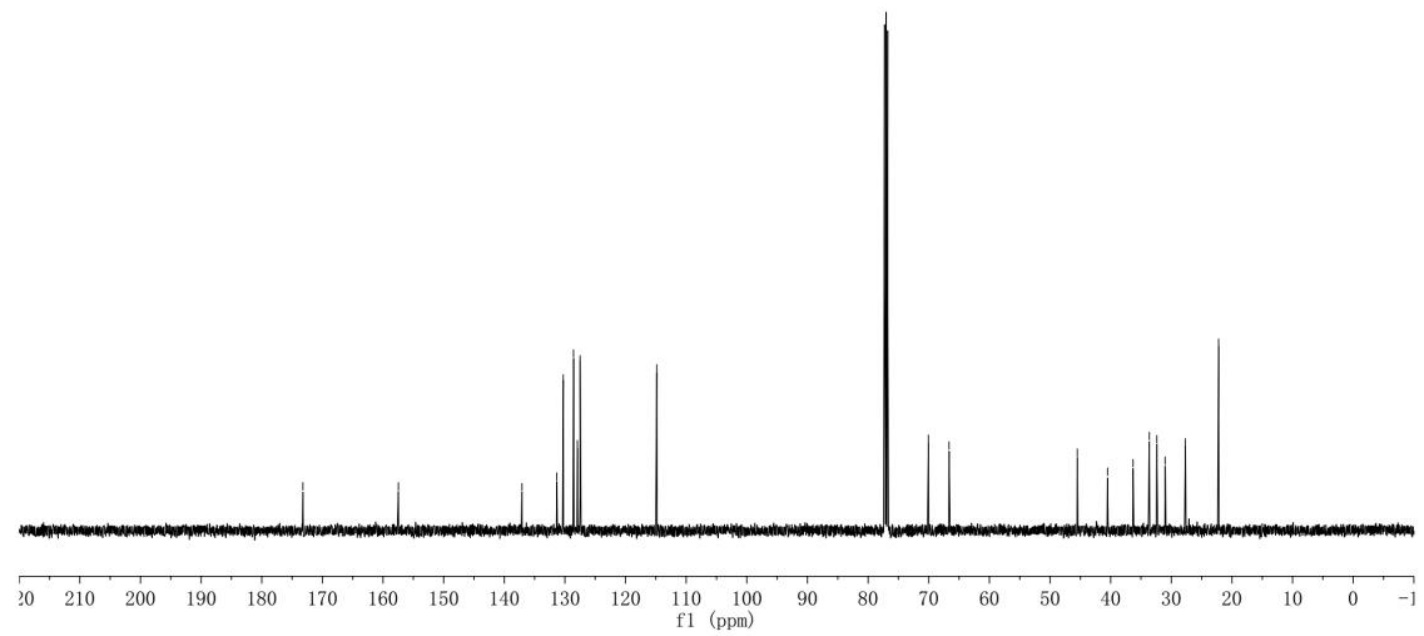




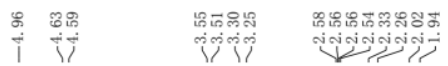

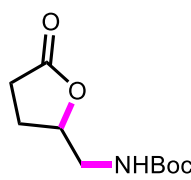

$7 a$

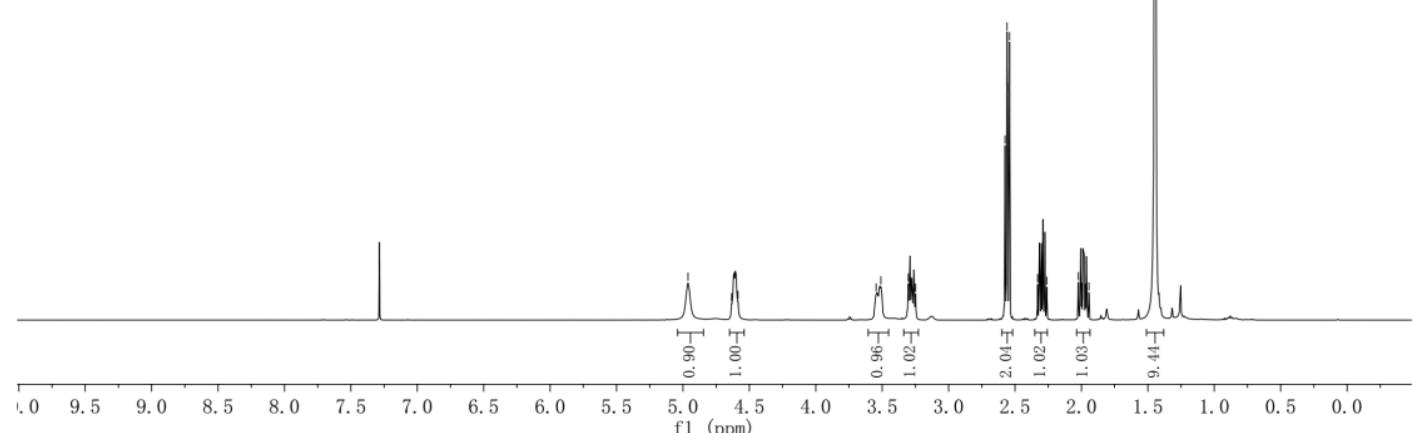

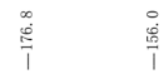

然

0
$\dot{1}$

\begin{tabular}{lllllllllllllllllllllllllllllll}
\hline 0 & 210 & 200 & 190 & 180 & 170 & 160 & 150 & 140 & 130 & 120 & 110 & 100 & 90 & 80 & 70 & 60 & 50 & 40 & 30 & 20 & 10 & 0 & -1
\end{tabular} 


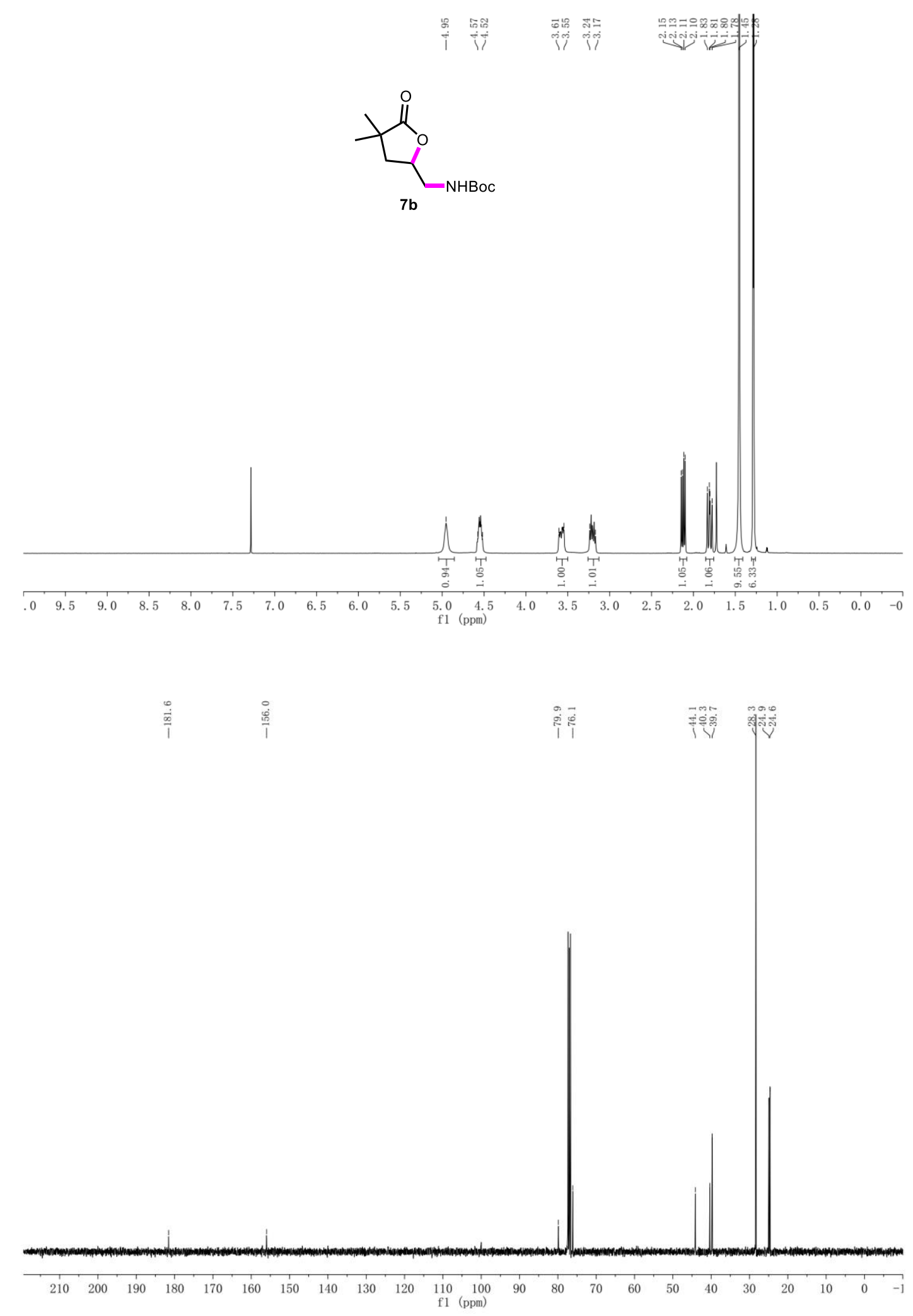



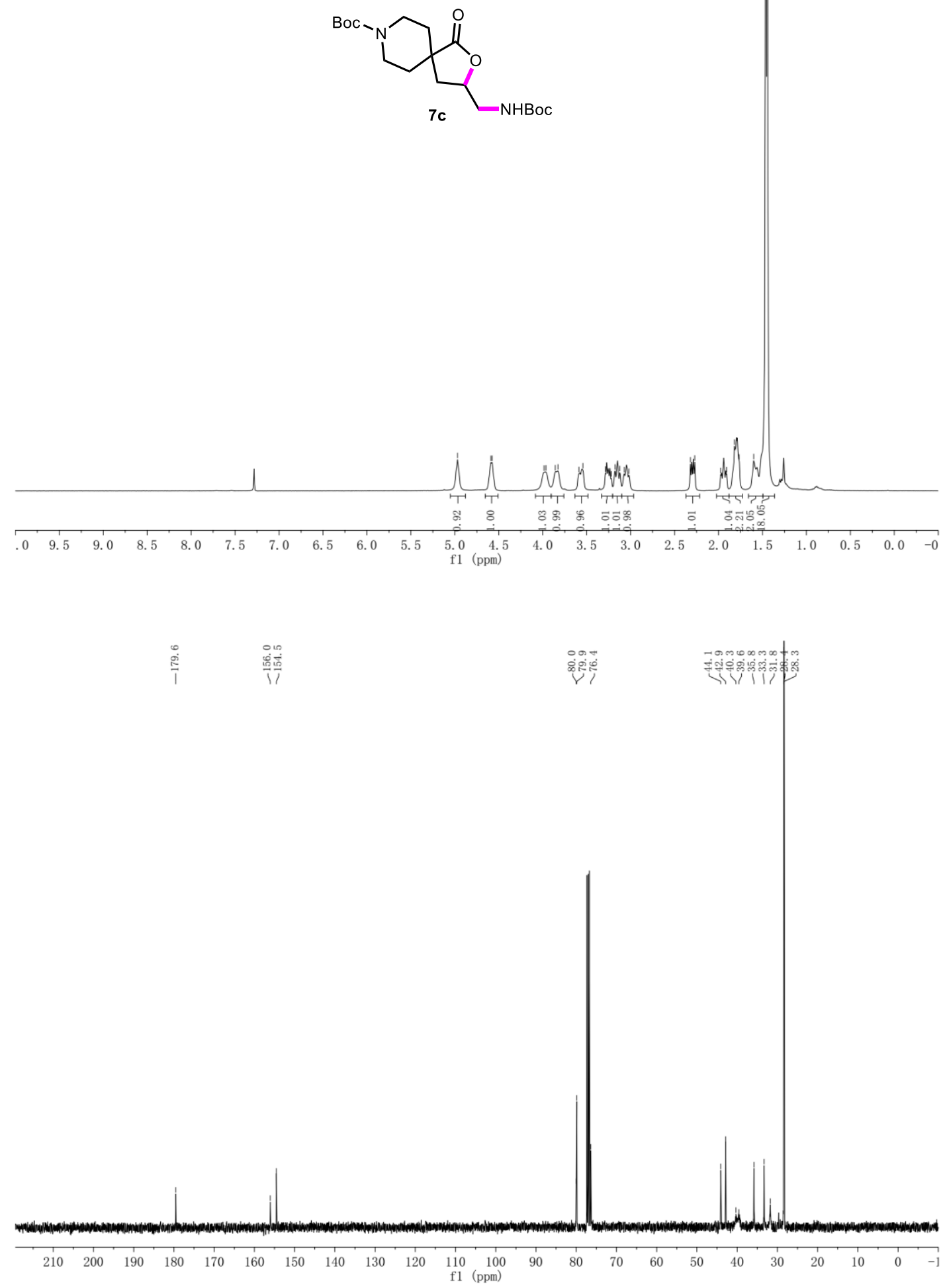

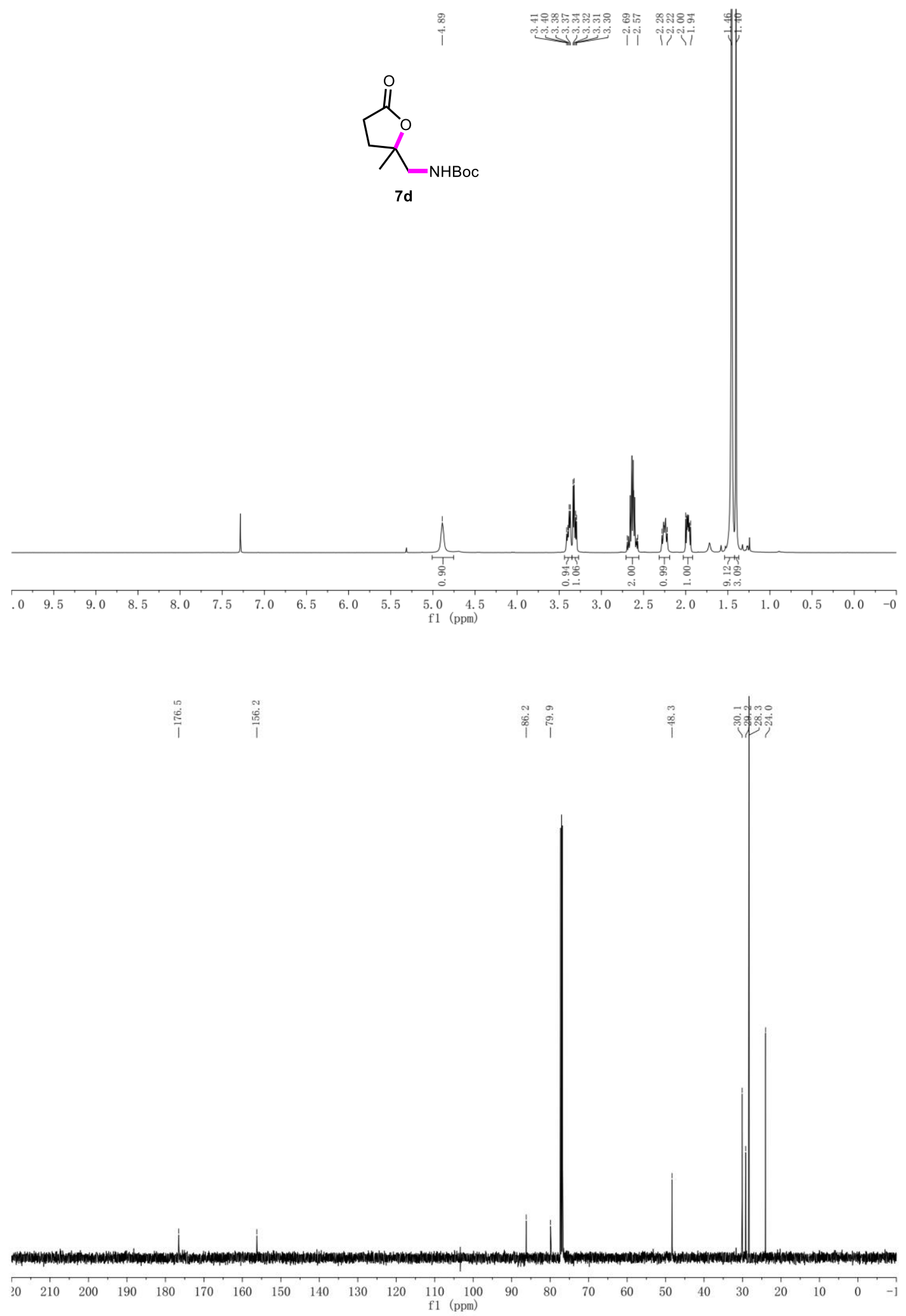


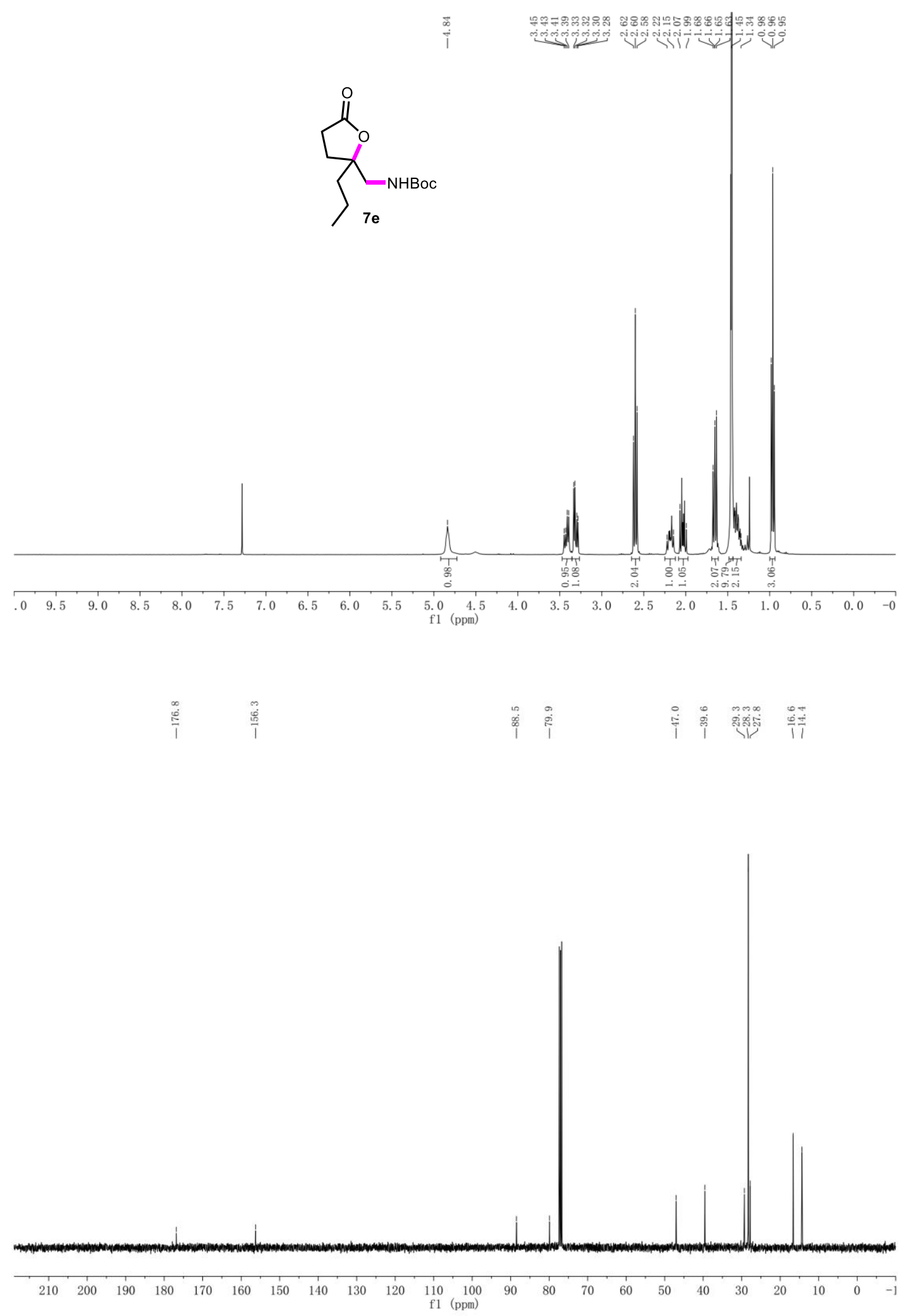




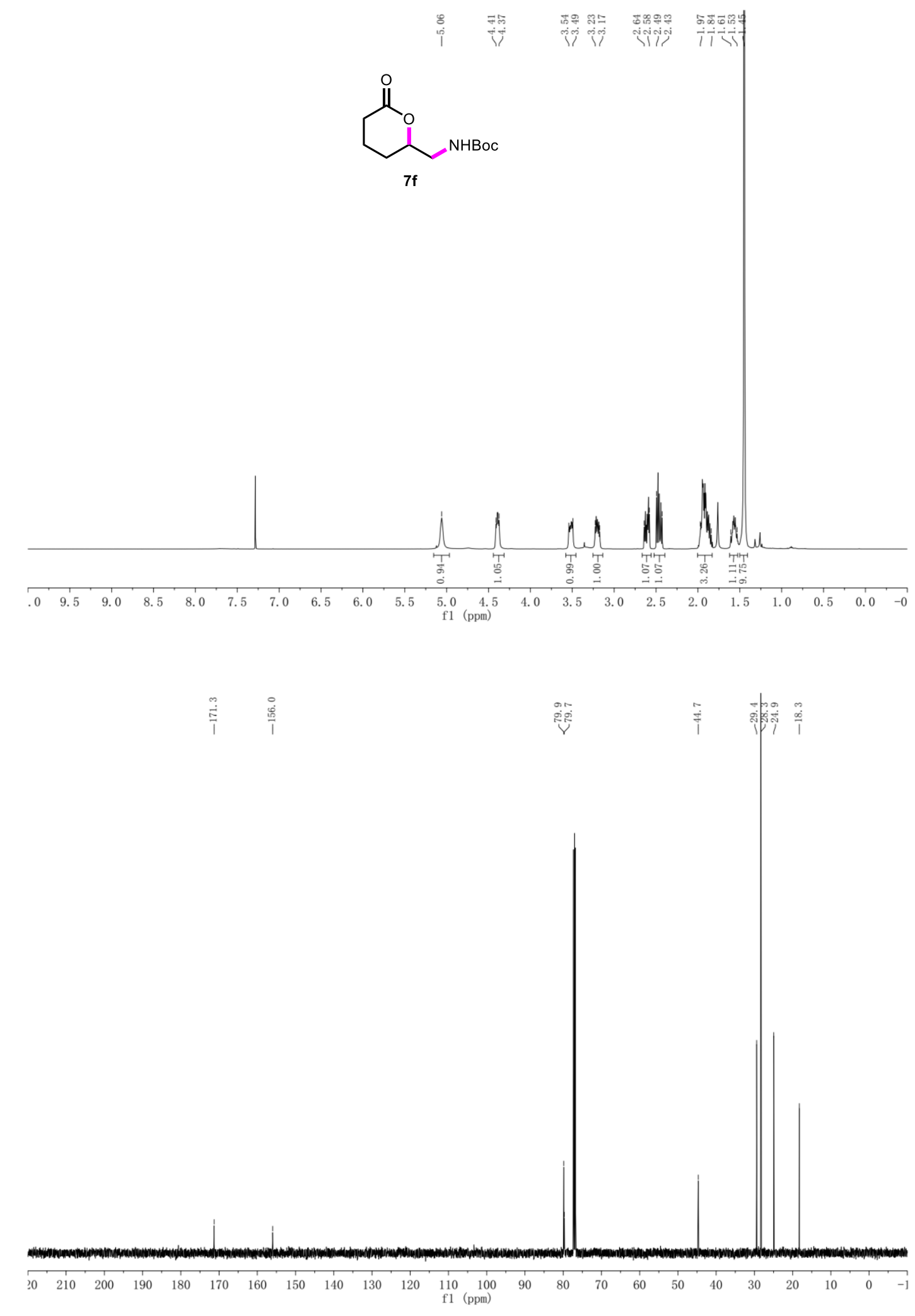



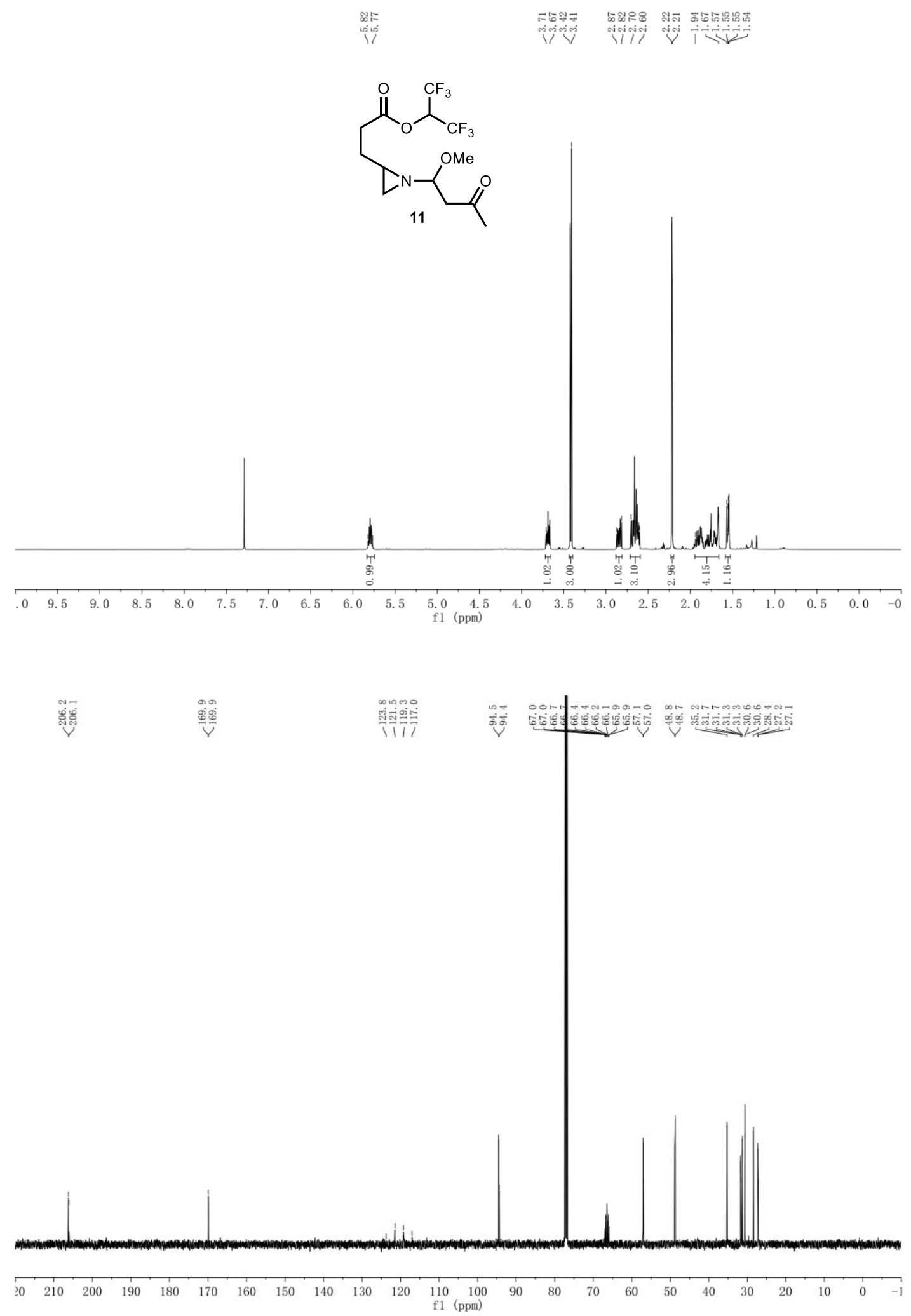
${ }^{19}$ F NMR<smiles>CC(=O)CC(O)N(C(=O)CCC(=O)OC(C(F)(F)F)C(F)(F)F)C1CC1</smiles>

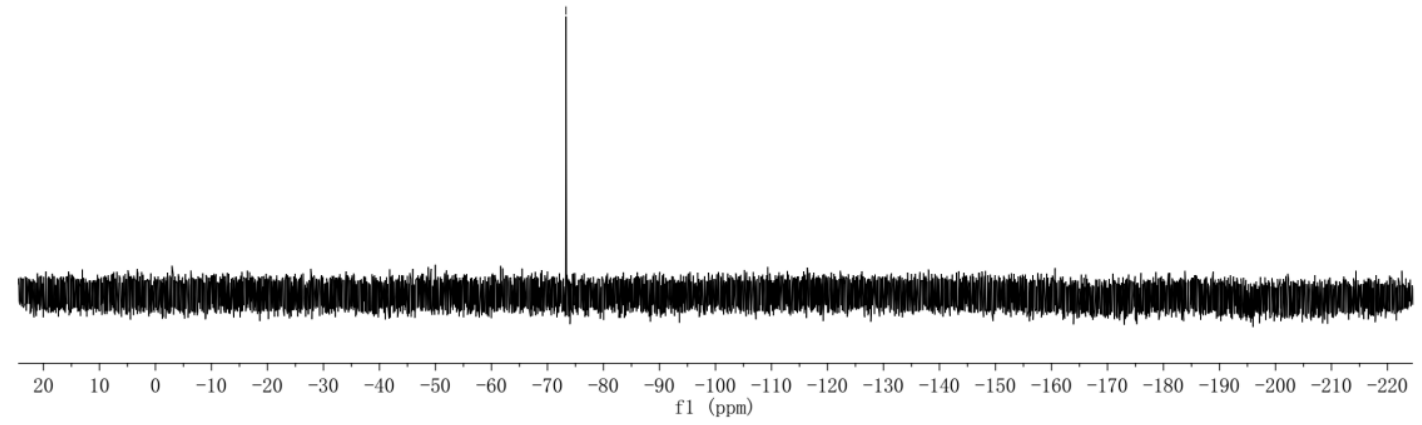

\section{COSY}

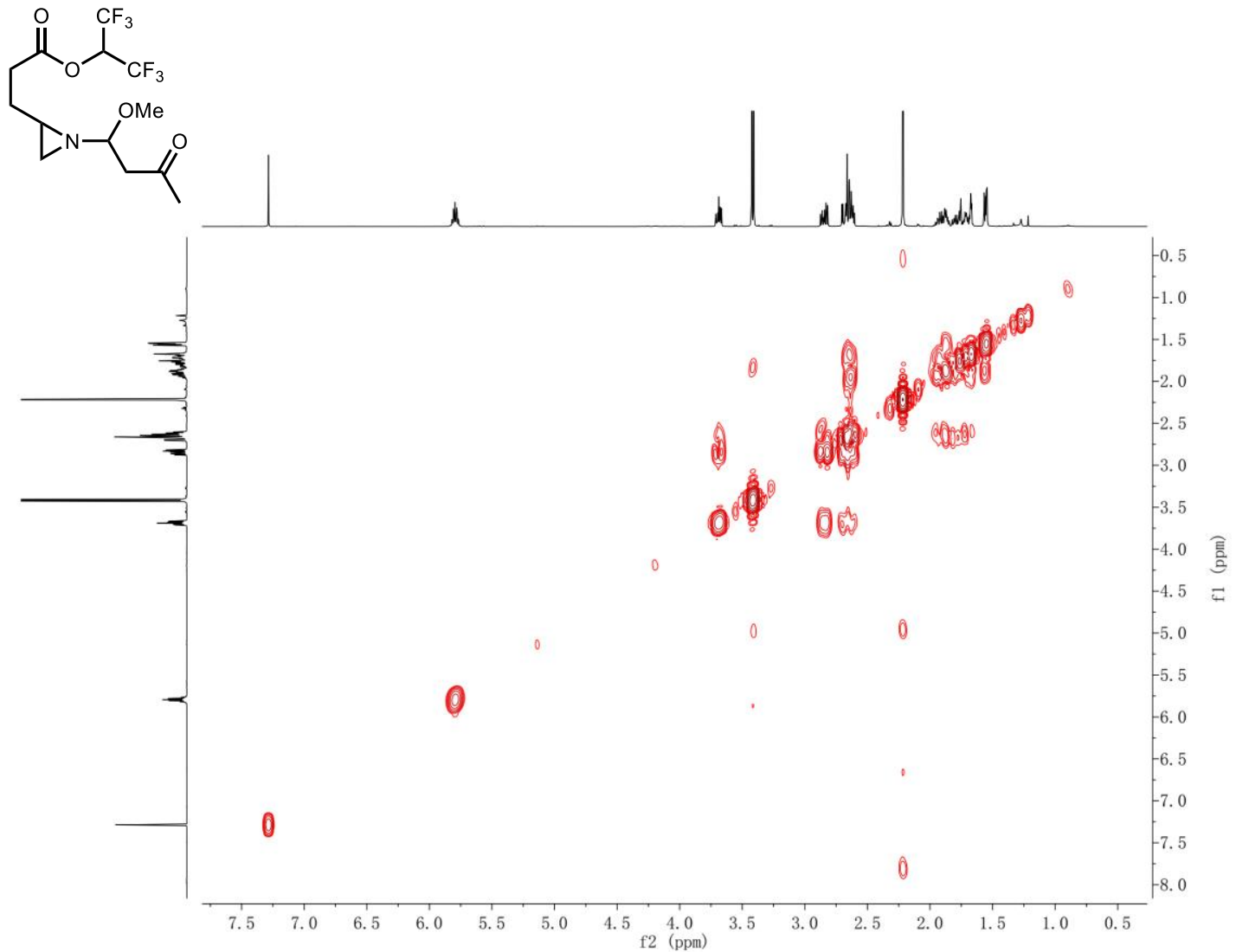



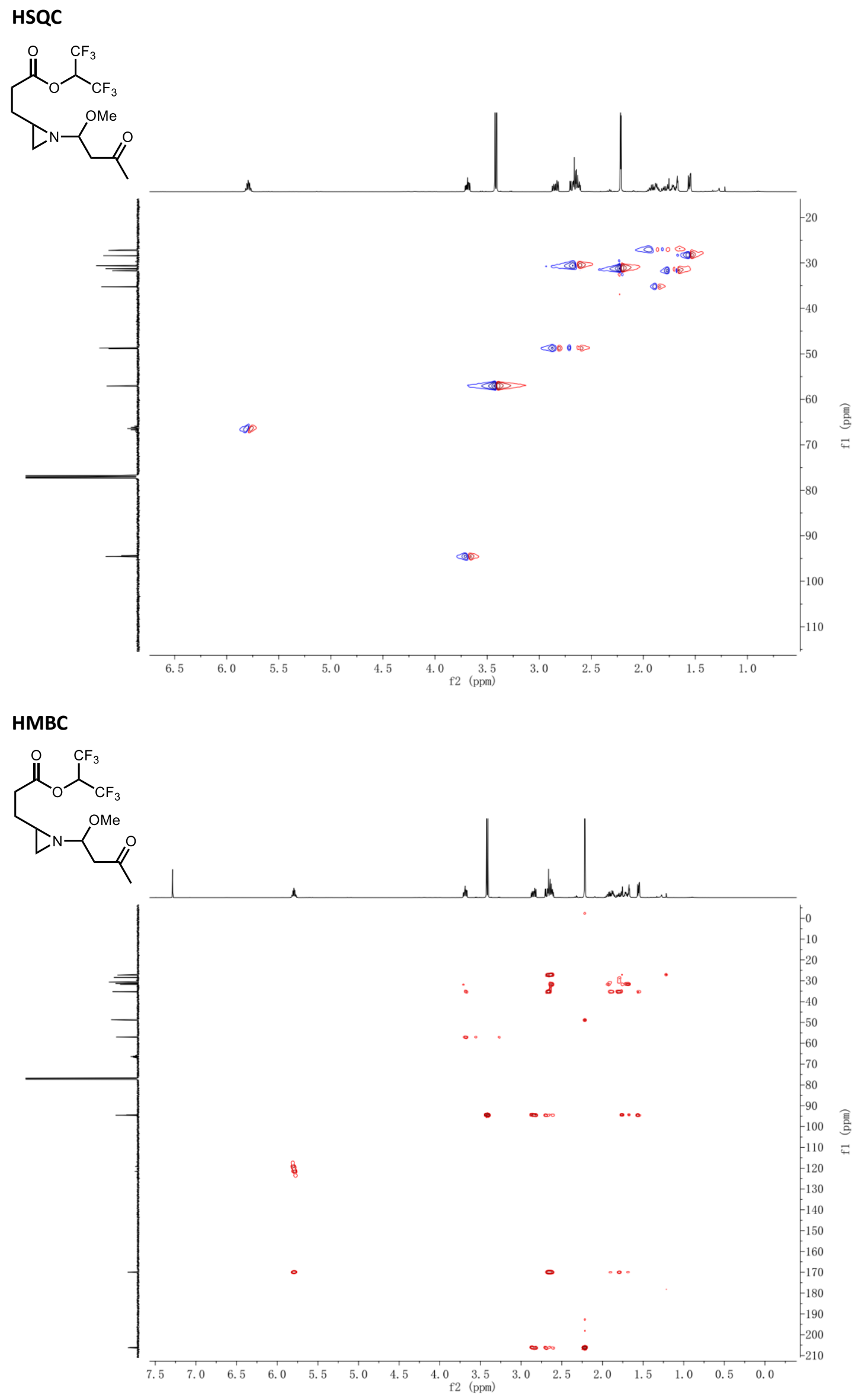

S75 


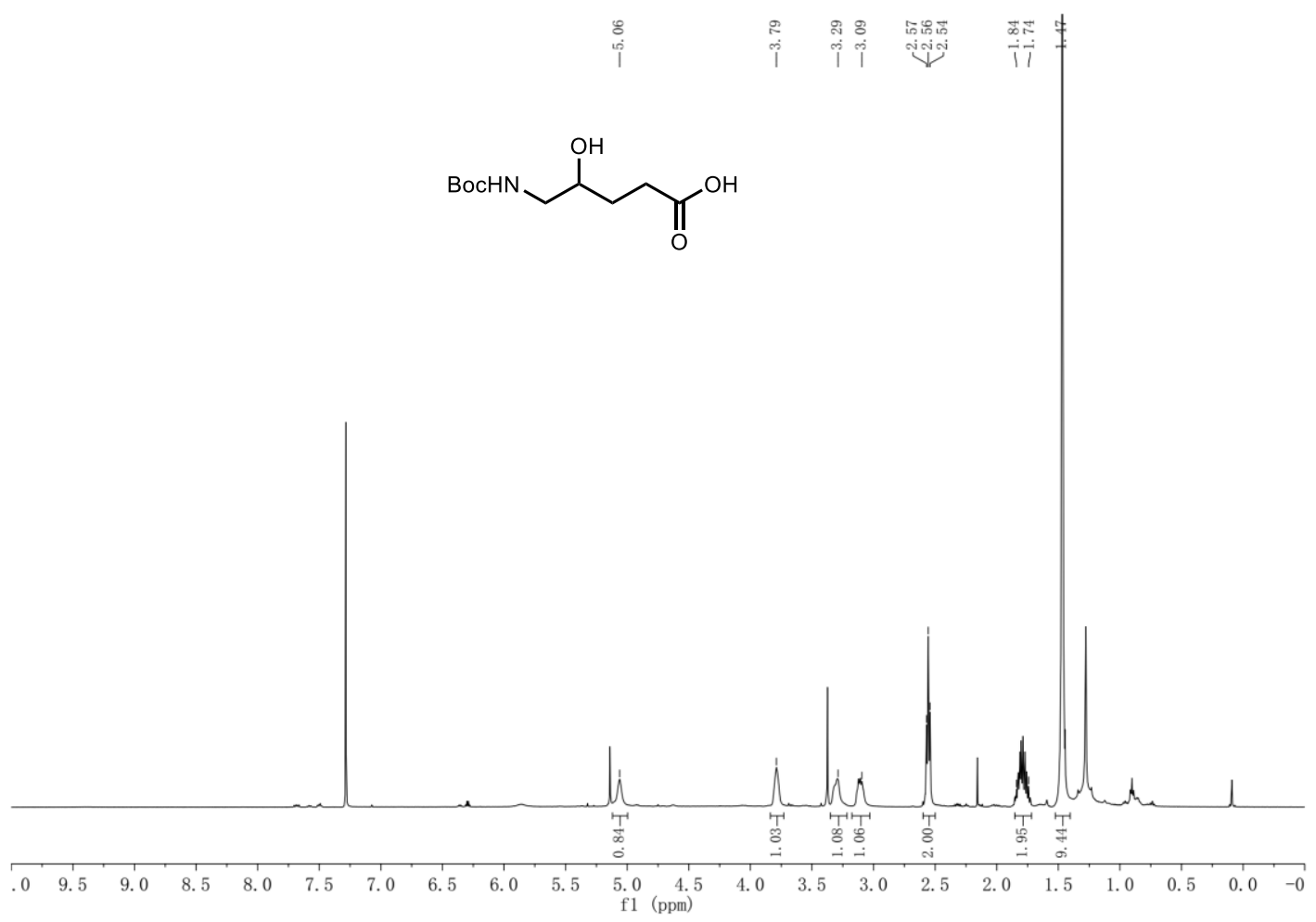

S76 